


\section{IMPACT EVALUATION BASELINE SURVEY OF SCHOOL DORMITORY ENVIRONMENT IN MONGOLIA}

Asako Maruyama, Nina Blöndal, Nyamdavaa Yondonjamts, Altantuya Tseveen, Turmunkh Purevsambuu, Narantsatsral Danzan, and Munkhjin Bayansan

DECEMBER 2019 
(C) 2019 Asian Development Bank

6 ADB Avenue, Mandaluyong City, 1550 Metro Manila, Philippines

Tel +632 8632 4444; Fax +63286362444

www.adb.org

Some rights reserved. Published in 2019.

ISBN 978-92-9261-954-1 (print), 978-92-9261-955-8 (electronic)

Publication Stock No. TCS190595-2

DOI: http://dx.doi.org/10.22617/TCS190595-2

The views expressed in this publication are those of the authors and do not necessarily reflect the views and policies of the Asian Development Bank (ADB) or its Board of Governors or the governments they represent.

ADB does not guarantee the accuracy of the data included in this publication and accepts no responsibility for any consequence of their use. The mention of specific companies or products of manufacturers does not imply that they are endorsed or recommended by $A D B$ in preference to others of a similar nature that are not mentioned.

By making any designation of or reference to a particular territory or geographic area, or by using the term "country" in this document, $A D B$ does not intend to make any judgments as to the legal or other status of any territory or area.

This work is available under the Creative Commons Attribution 3.0 IGO license (CC BY 3.0 IGO)

https://creativecommons.org/licenses/by/3.0/igo/. By using the content of this publication, you agree to be bound by the terms of this license. For attribution, translations, adaptations, and permissions, please read the provisions and terms of use at https://www.adb.org/terms-use\#openaccess.

This CC license does not apply to non-ADB copyright materials in this publication. If the material is attributed to another source, please contact the copyright owner or publisher of that source for permission to reproduce it. ADB cannot be held liable for any claims that arise as a result of your use of the material.

Please contact pubsmarketing@adb.org if you have questions or comments with respect to content, or if you wish to obtain copyright permission for your intended use that does not fall within these terms, or for permission to use the ADB logo.

Corrigenda to ADB publications may be found at http://www.adb.org/publications/corrigenda.

Notes:

In this publication, “\$” refers to United States dollars.

ADB recognizes "China" as the People's Republic of China.

The school year in Mongolia starts on 1 September and ends on 1 June.

"SY" before a calendar year denotes the year in which the school year starts, e.g., SY2016 ends on 1 June 2017.

Cover design by Mike Cortes. 


\section{CONTENTS}

TABLES AND FIGURES

CURRENCY EQUIVALENTS

viii

ABBREVIATIONS

viii

GLOSSARY

viii

ABSTRACT

ACKNOWLEDGMENTS

I INTRODUCTION

II PROJECT BACKGROUND AND OBJECTIVES

A I DORMITORIES IN MONGOLIA 3

B I PROJECT AREA

C I PROJECTINTERVENTIONS

D I PROJECT OUTCOMES AND THEORY OF CHANGE

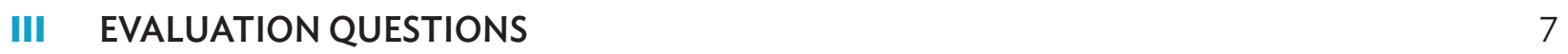

A I RELATED RESEARCH

B I EVALUATION QUESTIONS AND HYPOTHESES

IV APPROACH AND METHODOLOGY 9

$\begin{array}{ll}\text { V BASELINE SURVEY FINDINGS } & 10\end{array}$

\begin{tabular}{l|l} 
A I SURVEY RESPONDENTS & 10
\end{tabular}

B I PHYSICAL DORMITORY ENVIRONMENT 15

C I CAPACITY TO DELIVER DORMITORY SERVICES 23

D I ADVANCED AND FINAL OUTCOMES 33 
VI CONCLUSION AND RECOMMENDATIONS

A I BACKGROUNDS OF THE RESPONDENTS

B I PHYSICAL SCHOOL DORMITORY ENVIRONMENT 38

C I CAPACITY TO DELIVERY DORMITORY SERVICES

D I ADVANCED AND FINAL OUTCOMES 39

$\begin{array}{ll}\text { E I RECOMMENDATIONS } & 40\end{array}$

$\begin{array}{ll}\text { REFERENCES } & 41\end{array}$

\section{ANNEXES}

1 Project Overview $\quad 43$

2 Theory of Change $\quad 45$

3 Summary of Related Literature 46

4 Approach and Methodology 53

5 Year of Dormitory Construction and Renovation 64

6 Vignettes $\quad 67$

7 Selected Indicators across Treatment and Comparison Groups 69

8 Photos $\quad 71$ 


\section{TABLES AND FIGURES}

\section{TABLES}

1 Average Construction Year of Dormitories 16

2 Dormitories with Previous Rehabilitation 16

3 Average Year of Last Rehabilitation 16

A1 Dormitory Sample by Treatment Status and by Aimag 57

A2 Overview of Sample by Survey Type and by Treatment Group 57

A3 Survey Tools $\quad 59$

A4 Year of Construction: Full Treatment 63

A5 Year of Renovation: Full Treatment 63

A6 Year of Construction: Light Treatment 64

A7 Year of Renovation: Light Treatment 64

A8 Year of Construction: Comparison $\quad 65$

A9 Year of Renovation: Comparison $\quad 65$

A10 Prevalence of Selected Indicators across Treatment and Comparison Groups at Baseline 68

\section{FIGURES}

1 Female Staff, by Position 11

2 Educational Attainment of Dormitory Guards 11

3 Educational Attainment of Dormitory Teachers 11

4 Educational Attainment of Class Teachers 12

5 Teaching Qualifications of Class Teachers 12

6 Parents Who Were Interviewed 13

7 Educational Attainment of Mother 13

8 Educational Attainment of Father 13

9 Mother's Employment $\quad 14$

10 Father's Employment $\quad 14$ 
11 Mean Income of Parents 15

12 Income Sources of Parents $\quad 15$

13 Students' Experiences in a Dormitory Environment 17

14 Latrine Availability and Suitability for Early Graders and for Children with Disabilities 18

15 Dormitories with Outdoor Latrine and Suitability to Vulnerable Groups 18

16 Toilet Facilities for Girls $\quad 19$

17 Fear of Going to the Toilet at Night, by Grade 19

18 Hygiene Practices of Dormitory Students 20

19 Student Hygiene Behavior: Frequency of Washing Hands after Going to the Toilet 20

20 Student Hygiene Behavior: Taking a Shower Once a Week at Dormitory 20

21 Availability of Dormitory Furnitures Based on Enumerator's Observation 21

22 Dormitory Facilities $\quad 21$

23 Common Room with Equipment 22

24 Study Space in the Dormitory $\quad 22$

25 Students' Response to "What bothers you when you want to do your homework, 23 study, read, and concentrate?"

26 Presence of Any Kind of Fire Extinguisher in the Dormitory 23

27 Dormitory Teachers Who Received Training, by Topic 24

28 Dormitory Guards Who Received Training, by Topic 25

29 Cooks Who Received Training, by Topic 25

30 Teachers Reporting Having a Plan to Work with Parents, by Group 25

31 Parents' Observation of School-Organized Activities 26

32 Parents' Participation in School-Organized Activities 26

33 Dormitory Committees and Activity 27

34 Students Engaging in After-School Activities or Clubs 27

35 Reasons for Not Attending After-School Activities 28

36 Average Number of Meals Served Daily 28

37 Average Number of Daily Ingredients 28

38 Cooking Ingredients by Frequency of Use 29

39 My Parents Are Interested in My School Activities 29

40 My Parents Support My Educational Efforts and Achievements 30

41 My Parents Support Me When I Am Facing Difficulties at School 30

42 Average Weekly Hours of Parental Support to Their Children 30 
43 Students' Response to "How well do you like your school dormitory?" 31

44 Students' Self-Rating on Their Happiness and Well-Being 31

45 Dormitory Students Reporting Being Sick in the Last 2 Weeks 32

46 Number of Sick Days for Those Who Were Sick in the Last 2 Weeks 32

47 Reasons for Placing Child in a Dormitory 33

48 Students Missing at Least 1 School Day in the Last 2 Weeks 34

49 Frequency of Missing School in the Last 2 Weeks 34

50 Children Who Were Late for School in the Last 2 Weeks 34

51 Children Who Did Their Homework Every Day 34

52 Average 5th Grade Test Scores of Students Staying in Dormitories 35

53 Students Obtaining “Excellent” or "Good” Scores: Students Staying in Dormitories, Sample Only 35

54 Students Obtaining "Excellent” or "Good” Scores: All 5th Grade Students in Survey Aimags 36

A1 Distribution of Students Staying in Dormitories by Grade and \% of Students Interviewed 58 


\section{CURRENCY EQUIVALENTS}

as of 29 April 2018

$\begin{array}{ll}\text { Currency unit } & \text { togrog }(M N T) \\ \text { MNT1.00 } & \$ 0.00042 \\ \$ 1.00 & \text { MNT2,401.65 }\end{array}$

\section{ABBREVIATIONS}

$\mathrm{ADB}$

MECSS

PSM

$\mathrm{RCT}$

SIF

TOC

WASH
Asian Development Bank

Ministry of Education, Culture, Science and Sports

propensity score matching

randomized controlled trial

Social Investment Fund

theory of change

water, sanitation, and hygiene

\section{GLOSSARY}

aimag

ger

soum an administrative unit equivalent to a province in Mongolia

a traditional tent where seminomadic herders live

subunit of an aimag 


\section{ABSTRACT}

$T^{1}$

his report presents the impact evaluation of interventions supported under a grant project in Mongolia that was administered by the Asian Development Bank during 2016-2019. It also describes the methodology of the impact evaluation and discusses the findings of the baseline survey conducted during the school year 2016/17. The baseline survey examined the situation surrounding the dormitories, schools, and respondents in full and light treatment, and comparison groups prior to the start of the project. The survey focused on the (i) physical school dormitory environment; (ii) capacity to deliver dormitory services; and (iii) some of the outcomes, including parents' attitude in placing their children in a dormitory; school attendance among students living in dormitories; their level of participation in study, reading, and extracurricular activities; and 5th grade students' test scores in math, Mongolian language, and science. The baseline survey indicates selection bias-such as differences in student characteristics and physical dormitory environment - which should be mitigated through econometric techniques before the conduct of the endline survey during school year 2020/21.

Keywords: impact evaluation, quasi-experimental approach, matched difference-in-differences, school dormitories, primary education, Mongolia. 


\section{ACKNOWLEDGMENTS}

The authors wish to thank those who supported the production of this report.

The Ministry of Education, Culture, Science and Sports of the Government of Mongolia, especially, Myagmar Jadambaa and Tsolmon Ariya, provided overall guidance and support for the impact evaluation.

From the Asian Development Bank, Amy Leung, director general, M. Teresa Kho, deputy director general, Ying Qian, advisor, and Sangay Penjor, director, East Asia Department, guided the production of this report. The report benefited from the valuable comments provided by Ryotaro Hayashi, social sector economist, Elisabetta Gentile, economist, and Akiko-Terada Hagiwara, principal economist. Ruth D. Benigno, associate project analyst, coordinated the publication. Alvin Tubio, consultant, did the layout and typesetting.

Finally, the authors acknowledge financial support for the detailed design of the impact evaluation and the conduct of the baseline survey on which this report was based, from the technical assistance (TA 8332, Subproject 1): Developing Impact Evaluation Methodologies, Approaches, and Capacities in Selected Developing Member Countries. 


\section{INTRODUCTION}

$\mathrm{H}^{2}$ ow does a poor school dormitory environment affect parents' decision to send young childrenaged 6 or 7-to school? How does living in a dormitory affect the students' social adjustment, psychological well-being, and learning outcomes? How do poor school dormitory services influence the level of students' participation in study, reading, and extracurricular activities; and their learning outcomes? What intervention or combination of interventions would work best to increase participation in study, reading, and extracurricular activities among students living in dormitories and improve their learning outcomes?

The Asian Development Bank (ADB) administered a grant assistance to the Government of Mongolia provided by the Japan Fund for Poverty Reduction during 2016-2019 with the aim of improving the school dormitory environment for primary students in one of the poorest regions in Mongolia. ${ }^{1}$ The project was developed due to a changing policy environment surrounding school dormitories in Mongolia.

During the period 2012-2016, the school dormitory system was considered as part of the learning environment in the education sector reform policy framework and was identified as one of the 11 reform areas to ensure equity of access to quality education. ${ }^{2}$

The project targeted three out of five aimags in the western region of Mongolia-Govi-Altai, Uvs, and Zavkhan. ${ }^{3}$ It supported the development and piloting of models to improve the school dormitory environment-with a view to replicating the models nationwide-by designing and implementing three groups of interventions. These are (i) improving the physical school dormitory environment, (ii) strengthening the capacity to deliver school dormitory services, and (iii) developing policy and regulatory frameworks to improve the school dormitory environment.

This report (i) describes the methodology to evaluate the impact of the project interventions, particularly physical school dormitory upgrade and staff capacity development, and (ii) discusses the findings of the baseline survey conducted during school year (SY) 2016/17. ${ }^{4}$ The baseline survey aimed to clarify the causal pathways of each project intervention to the expected outputs and outcomes.

1 ADB. 2015. Report and Recommendation of the President to the Board of Directors: Proposed Administration of Grant to Mongolia for Improving School Dormitory Environment for Primary Students in Western Region Project. Manila.

2 Ministry of Education, Culture, Science and Sports. 2012. Pre-School, Primary and Secondary Education Reform Policy Framework, 2012-2016. Ulaanbaatar.

3 Aimag is an administrative unit equivalent to a province in Mongolia. There are 21 aimags in the country.

4 The report benefited from the valuable comments provided by Ryotaro Hayashi, social sector economist, and Elisabetta Gentile, economist, both of ADB. 
It also assessed the situation surrounding the project and comparison areas prior to starting the project interventions. Based on survey findings, this report provides recommendations for preparing an endline survey to measure outcomes of the project interventions, to be conducted in SY2020/21.

The impact evaluation is intended to contribute to the improvement and refinement of nationally replicable models to (i) improve school dormitory environment, (ii) help inform the development of comprehensive standards for the physical dormitory environment and services, and (iii) support the development of a national strategy and financing policy to improve school dormitories. It is also intended to (i) fill a knowledge gap on factors that affect parents in remote rural areas and their decision to send their children to school; and (ii) determine the attitudes and levels of participation in study, reading, and extracurricular activitiesand learning outcomes-among students living in dormitories. 


\section{PROJECT BACKGROUND AND OBJECTIVES}

M ongolia is a sparsely populated country with livestock outnumbering the population. Seminomadic herders make up a substantial, albeit decreasing, proportion of the economically active population that accounted for $24.4 \%$ in 2016.

\section{A | DORMITORIES IN MONGOLIA}

Mongolia's school dormitory system was developed to ensure access to education-particularly for students from herder families — and has been supported by special provisions in the Education Law since 1982. Until 1995, dormitories were fully funded by the Government of Mongolia and were a contributing factor in the country's high levels of enrollment in primary and secondary education. However, the introduction of fees for dormitory meals and other services in 1995, which was made in response to cutbacks in public spending on education - while in the process of transition from a centrally planned to a market economyresulted in a decreased number of students staying in dormitories and enrollments in primary and secondary education. Following the resumption of full government funding of dormitory services in 2000 , both the number of students staying in dormitories and enrollments increased considerably.

The chronically low level of capital investment in the education sector led to the deterioration of the physical environment of school dormitories. In SY2016/17, there were 513 functional dormitories in Mongolia, of which 99 (19\%) did not meet the national school building standards. Many of these dormitories were built in the 1970s and 1980s and have become unfit to accommodate students. Although public spending on education in Mongolia has been higher than in other lower middle-income countries, a large share has been devoted to covering relatively high recurrent costs due to the country's low population density and harsh winters. Schools tend not to spend money on dormitories because school budgets do not distinguish funding for dormitories from other budget items, except dormitory staff salaries and dormitory meals. As a result, dormitories often lack safe drinking water, adequate sanitation facilities, heating systems, and protection from precipitation and wind. The situation of water, sanitation, and hygiene (WASH) in dormitories is further complicated by the absence of basic infrastructure for water, wastewater, and heating in remote rural areas. 


\section{B | PROJECT AREA}

The project targeted three out of five aimags in the western region of Mongolia-Govi-Altai, Uvs, and Zavkhan. The western region was one of the poorer regions in the country, with a poverty head count ratio that is substantially higher (36.0\% in 2016) than the national average (29.6\%). Of the five regions in Mongolia, the western region had the largest number of students staying in dormitories $-12,244$ in SY2016/17-which corresponds to $14.5 \%$ of students enrolled in primary and secondary education. The vast majority - 78.8\% - came from herder families. Herders comprised more than $45 \%$ of the economically active population in the region in 2016 and constituted a substantial share of the poor at $36 \%$.

In SY2016/17, the primary completion rate in the western region was $90.2 \%$, which was substantially below the national average of $93.5 \%$, and the lowest of all the regions. Children in the region tend to start school later than the official school age of 6 , as evidenced by the lowest net intake rate in 1st grade of all the regions, which was $83 \%$ in 2016, as opposed to the national average of $94.9 \%$. A study conducted in 2008 revealed that students staying in dormitories and those who entered school at the age of 7 or 8 demonstrated lower academic performance than their peers who entered school at age 6. Unfavorable school dormitory environment, lack of parental support, and the children's difficulties in adjusting to school and dormitory would probably explain the findings.

Early-grade students staying in dormitories require special care and support as they often have difficulties in adjusting and making a smooth transition from home to school. This situation is especially true among students from herder families. Since schools and dormitories are the place where they are first exposed to other groups, their sense of disorientation could be more acute than the other students. The dropout rate for the 1st grade students is usually higher than those for other grade students. ${ }^{6}$ Even if students need to stay in dormitories as schools are located far from home, due to unfavorable dormitory environment, they sometimes end up not opting for dormitories. In 2014, $9 \%$ of students who needed to stay in dormitories did not choose to stay in dormitories. In 2015, less than 30\% of primary students in dormitories regularly participated in study, reading, and extracurricular activities in dormitories-and this was probably due to high student-teacher ratios in dormitories, and lack of qualifications and skills among dormitory teachers and staff. These issues negatively affect parents' decision to send children to school, especially at early ages, in rural remote areas.

5 Education Evaluation Center of Mongolia. 2008. Mongolian National Assessment of Primary Education Mathematics and Reading. Ulaanbaatar.

6 The dropout rate for 1 st grade students was 2\%, and the repetition rate was 0.14\% during SY2012/13-SY2013/14, according to data provided by the MECSS. 


\section{C | PROJECT INTERVENTIONS}

The project ${ }^{7}$ supported the following interventions, which aimed to improve the school enrollment of children aged 6 , their well-being, and their learning:

(i) Upgrading of physical school dormitory environment (February 2018-September 2019)

(a) Rehabilitate dormitories to cope adequately with winter temperatures, wind, and precipitation.

(b) Equip dormitories with WASH facilities that meet the minimum requirements of the Ministry of Education, Culture, Science and Sports (MECSS) and incorporate features responsive to gender and early-grade students.

(c) Renovate and equip common rooms for study, reading, and extracurricular activities.

(ii) Training of dormitory staff (March 2018-September 2019)

(a) Train dormitory teachers in child abuse issues and in child-centered methods to facilitate study, reading, and extracurricular activities and improve school preparedness of early-grade students.

(b) Train dormitory guards in child abuse issues and in child-centered methods to encourage students' participation in study, reading, and extracurricular activities and take care of students, especially among early graders, at night.

(iii) Promotion of student committee activities and student-organized study, reading, and extracurricular activities in dormitories among school management and teachers (March 2018-September 2019)

(iv) Promotion of outreach activities for parents with children living in dormitories, particularly those at the primary level, among school management and teachers (March 2018-September 2019)

\section{D | PROJECT OUTCOMES AND THEORY OF CHANGE}

In designing the impact evaluation, a working theory of change (TOC) was developed, in collaboration with the MECSS. The TOC (Annex 2) illustrates the intended causal pathways of each project intervention to achieve immediate outputs, which would further contribute to intermediate outcomes. The intermediate outcomes are then expected to lead to advanced and final outcomes, which are the focus of the impact evaluation, and these include the following:

parents' attitude in placing children in dormitory;

school attendance among students living in dormitories;

participation in study, reading, and extracurricular activities among students living in dormitories; school enrollment of children aged 6, measured through the net intake rate in 1st grade;

More detailed description of the project is presented in Annex 1. 
dropout rates;

gross intake rate in 5 th grade (last grade) of primary education;

5th grade test scores;

$\square$ primary completion rate; and

$\square$ junior secondary transition rate.

The TOC was used in the process of designing the impact evaluation to (i) map out and understand how the project interventions are intended to work, (ii) ensure that the surveys measure results along the entire causal chain, and (iii) identify any weak links in the causal chain. 
$T^{\text {nes }}$ he impact evaluation was designed based on the (i) combined experiences of ADB and other development partners in the education sector; (ii) good practices in boarding school management in Japan and other countries; (iii) interviews with school staff and herder families; (iv) existing education sector studies in Mongolia; and (v) studies from other countries on the linkages between school building conditions, parental involvement in children's education, and students' academic performance.

\section{A | RELATED RESEARCH}

There were no studies that directly measured the effect of physical dormitory upgrade, dormitory staff training, or parental involvement, or which specifically tested the hypothesis for the impact evaluation.

According to Snilstveit et al. (2015) who conducted a systematic review of studies evaluating the impact of education programs on children's learning and school participation, there were seven studies that measured the effect of school infrastructure development projects on educational outcomes in low- and middle-income countries. Five of these studies were relevant to the present impact evaluation, of which two evaluated the impact of latrine construction and hygiene education at primary schools in India and Kenya. Three studies dealt with projects that developed or upgraded school infrastructure, such as school libraries. A summary of the seven studies is found in Annex 3.

The two hygiene infrastructure studies indicated a positive effect on school participation. The three studies on school infrastructure development or upgrading projects collectively showed a positive effect on school enrollment and attendance. Nevertheless, the limited number of studies does not allow broader generalization of the findings.

Five of the seven studies used cluster-randomized trials where interventions took place at the school or village level. Two studies (i.e., school sanitation and hygiene education project in India; school rehabilitation project in Georgia), on the other hand, adopted quasi-experimental approaches, or the difference-in-differences method.

In addition to the studies above, Yue et al. (2012) examined the impact of dormitory "life teacher" training project in the People's Republic of China through a small cluster-randomized trial. Life teachers (shenghuo laoshi) are the staff who oversee the lives of students living in dormitories in the People's Republic of China. The study measured outcomes such as health, behavior, and academic performance of students living in dormitories. Moderate improvements in health outcomes were reported among students in the treatment group, $16 \%$ were less likely to feel cold at night, and $2 \%$ were less likely to experience diarrhea after the project. 
Behavior similarly improved, with declining incidents of being late to class (18\%), and misbehaviors after class (78\%). The researchers suggest that improved communication between life teachers and students may be one factor behind the observed results. The study, however, found no measurable impact on other outcomes, such as body mass index-for-age Z-score, misbehaviors in classroom, and test scores.

\section{B | EVALUATION QUESTIONS AND HYPOTHESES}

This impact evaluation attempts to answer the following questions:

(i) What is the impact of improved physical school dormitory environment on parents' decision to send young children to school; the students' participation in study, reading, and extracurricular activities; their social adjustment and psychological well-being; and their learning outcomes?

(ii) What is the impact of an improved capacity to deliver school dormitory services on parents' decision to send young children to school; on students' participation in study, reading, and extracurricular activities; their social adjustment and psychological well-being; and their learning outcomes?

(iii) Which of these interventions or combination of interventions is sustainable and works best to increase the students' participation in study, reading, and extracurricular activities, and improve their learning outcomes:

(a) training of dormitory staff; (b) promotion of student committee activities and student-organized study, reading and extracurricular activities among school management and teachers; or (c) promotion of outreach activities among school management and teachers for parents with children living in dormitories?

(iv) How do interventions compare in terms of cost-effectiveness?

The following hypotheses will be tested in the impact evaluation study:

Improved physical school dormitory environment influences enrollment of children aged 6 in primary education, especially those from herder families, in rural remote areas.

Improved physical school dormitory environment facilitates social adjustment of students, especially in early ages, and improves their psychological well-being.

Improved physical school dormitory environment enhances test results of students living in dormitories at the end of primary education (5th grade).

Improved physical school dormitory environment reduces dropouts and increase primary completion rates.

Trained dormitory staff support and facilitate students' participation in study, reading, and extracurricular activities in a more effective way and improve their learning outcomes (e.g., test results at the end of primary education).

If dormitory student committees are strengthened to organize study, reading, and extracurricular activities, students participate more in study, reading, and extracurricular activities and their learning outcomes improve.

If parents are regularly informed of their children's learnings, students' participation in study, reading, and extracurricular activities increases and their learning outcomes improve.

Improved capacity to deliver school dormitory services reduces dropouts and increases primary completion rates. 


\section{APPROACH AND METHODOLOGY}

$T^{\text {tis }}$ his impact evaluation adopts a theory-based approach, using the TOC (Annex 2) and measuring not only impacts but also exploring how the project works at all stages-from inputs to outputs to outcomes and impact. ${ }^{8}$ It examines the causal effects of the project interventions by comparing project beneficiaries, the treatment group, to a similar comparison group. A key challenge is to identify a valid comparison group, if the treatment cannot be randomly assigned, by avoiding or eliminating selection bias, which occurs when the treatment group is systematically and statistically different from a given comparison group.

This impact evaluation uses a quasi-experimental approach, or the matched difference-in-differences method. This method estimates the average treatment effect on the treated by comparing treatment and comparison groups (first difference) over time (second difference)-by carrying out identical surveys prior to the start of interventions (baseline) and after the interventions end (endline). To further strengthen the likelihood that the parallel trend exists between the treatment and comparison groups, propensity score matching is used to match treatment and comparison dormitories and students on baseline characteristics before estimating difference-in-differences results. The matching will be carried out in both-during preparation for the endline survey and after endline data collection - to account for the attrition of some participants.

There were 78 school dormitories in the three project aimags. Of those, 24 dormitories were identified to participate in the physical upgrade, training, and promotion activities in the course of designing the project; they are referred to as the "full treatment" group. The selection was made based on the condition of dormitory buildings and facilities, the year of dormitory construction and/or rehabilitation, the number of primary students staying in dormitories, the existence of junior and senior secondary grades, and the lack of basic infrastructure (water, wastewater, and heating). The remaining 54 dormitories in the three aimags were to participate in training and promotion activities only; they are referred to as the "light treatment" group. There were 80 "comparison" dormitories-24 dormitories of which matched the selection criteria for 24 "full treatment" dormitories, and 56 randomly sampled dormitories-were identified from other aimags.

The baseline survey conducted during SY2016/17 (February-June 2017) covered 2,911 primary school students and their parents, school management, school teachers, dormitory teachers, guards, and cooks in 158 dormitories - of which 78 were in the project aimags and 80 were in the comparison aimags.

The endline survey is planned for SY2020/21.

8 More detailed description of the approach and methodology is presented in Annex 4. 


\section{BASELINE SURVEY FINDINGS}

$T^{\text {his }}$

his section provides an overview of the treatment and comparison dormitories and the main outcomes at baseline. The facts and figures discussed here were selected to best describe the situation and illustrate the key outcomes rather than to comprehensively display the data collected. They are presented for the full treatment, light treatment, and comparison groups to demonstrate and compare pre-project similarities and differences. As the treatment and comparison groups will be matched for greater comparability at endline, the findings here focus on pre-matching differences and similarities.

\section{A | SURVEY RESPONDENTS}

Survey respondents included aimag education administrators, school management staff, class teachers, dormitory teachers, dormitory guards, cooks, and primary students staying in dormitories and their parents. An overview of key respondent backgrounds, school and dormitory staff, and parents of primary students staying in dormitories, which are likely to influence student outcomes independent of the project, is discussed below.

\section{(i) School and Dormitory Staff}

The majority of school and dormitory staff at the full treatment, light treatment, and comparison dormitories were female. Meanwhile, school principals in the two treatment groups only had 33\% female in the full treatment group and $47 \%$ in the light treatment group (Figure 1). Almost all class teachers and cooks were female across all groups. Female dormitory teachers were almost similar at $71 \%-77 \%$ across all groups. Differences were slightly larger for dormitory guards - all guards in the full treatment group were womencompared to $79 \%$ in the light treatment group, and $84 \%$ in the comparison group.

Educational attainment of dormitory guards varied from primary education to bachelor's degree (Figure 2). The majority, however, had upper secondary education with the proportion highest in the full treatment group (69\%). The rest of the guards in the full treatment group had lower secondary education (27\%) or bachelor's degree (4\%). In the light treatment and comparison groups, close to one-fifth of guards had vocational degrees, while less than $5 \%$ had only primary education or bachelor's degree (except in the light treatment group, with $11 \%$ having bachelor's degree). Educational attainment of dormitory teachers was more uniform with over $90 \%$ having a bachelor's degree in all three groups (Figure 3 ). 
Figure 1: Female Staff, by Position

$(\%)$

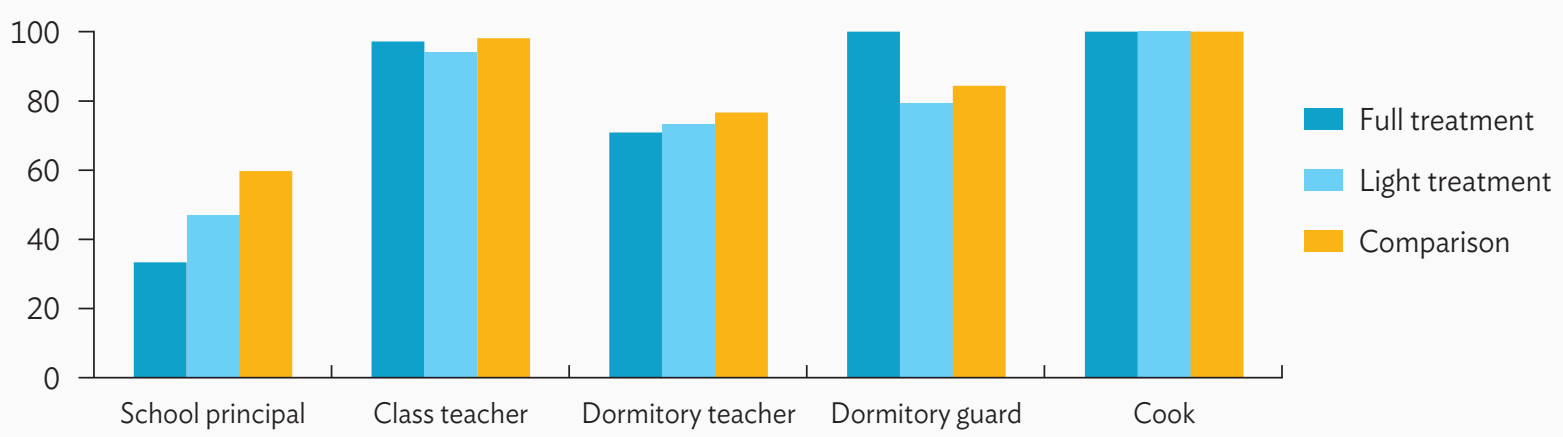

Source: Baseline survey conducted by the authors.

Figure 2: Educational Attainment of Dormitory Guards

$(\%)$

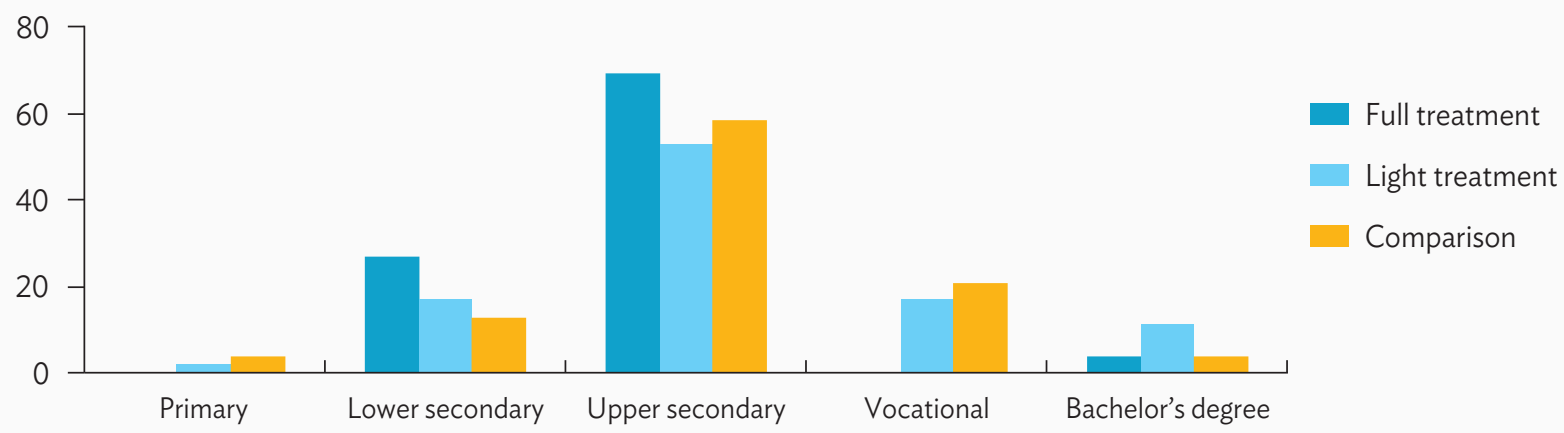

Source: Baseline survey conducted by the authors.

Figure 3: Educational Attainment of Dormitory Teachers

$(\%)$

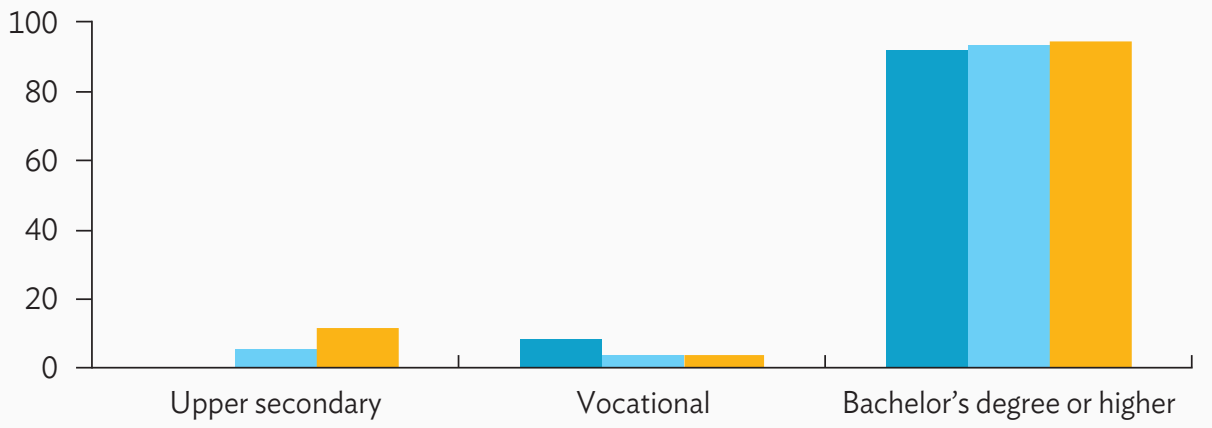

Full treatment

Light treatment

Comparison

Source: Baseline survey conducted by the authors. 
Over $80 \%$ of class teachers held a bachelor's degree, while a smaller percentage (5\%-13\%) obtained a master's or doctoral degree, and a still smaller proportion (5\%-7\%) attained a vocational degree (Figure 4). About $90 \%$ had a teaching license, and $60 \%$ also held a professional-level teaching qualification (Figure 5). Only small percentage variations existed among teachers across three groups.

Figure 4: Educational Attainment of Class Teachers

(\%)

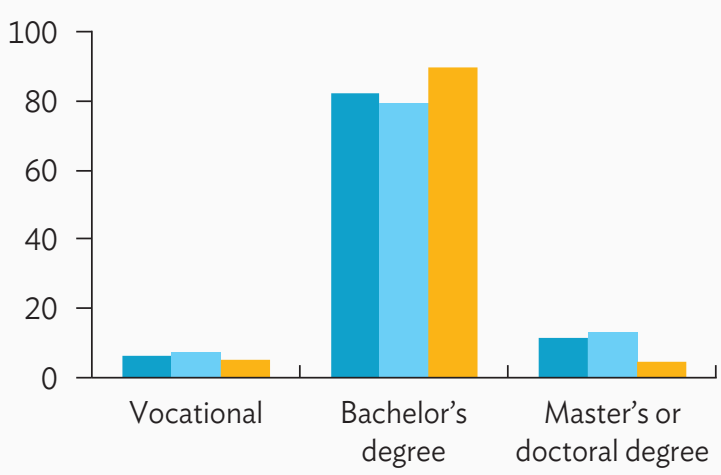

Full treatment

Light treatment

Comparison

Source: Baseline survey conducted by the authors.

\section{Figure 5: Teaching Qualifications of Class Teachers}

(\%)

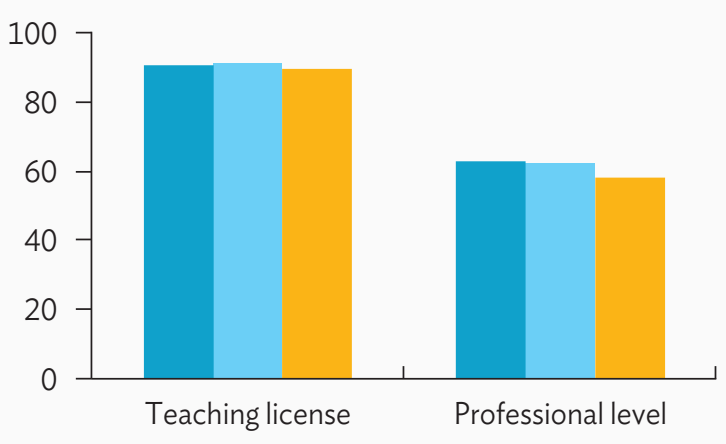

Full treatment

Light treatment

Comparison

Source: Baseline survey conducted by the authors.

\section{(ii) Parents of Students Living in Dormitories}

Parents' contact information was provided by the dormitories and interviews were conducted by phone. A total of 2,495 parents of students living in school dormitories were interviewed-70\% were mothers, $26 \%$ were fathers, and $4 \%$ were legal guardians (Figure 6 ). ${ }^{9}$

Of the respondents, about $15 \%$ of mothers and $20 \%$ of fathers did not go to school. Lower secondary was the most common level of educational attainment among mothers and fathers. The second most common level of attainment was upper secondary among mothers, and primary education among fathers (Figures 7 and 8). Parents in the comparison group were slightly better educated than the treatment groups, and those in the light treatment group were slightly better educated than the full treatment group. On average, mothers attained higher levels of education than fathers.

9 Interviews were conducted by phone. Only 2,176 parents of the 2,911 surveyed students living in dormitories could be reached for interview. To supplement those who could not be reached, 319 parents of students in the same dormitories, who were not covered by the survey, were also interviewed. 
Figure 6: Parents Who Were Interviewed

(\%)

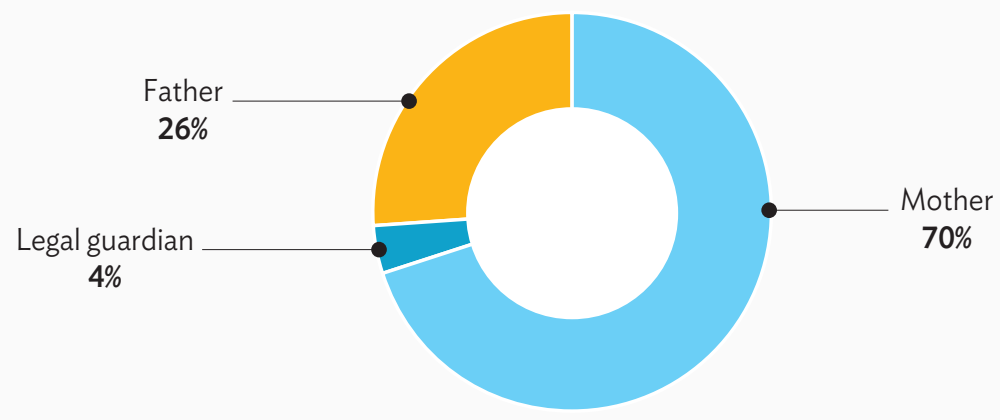

Source: Baseline survey conducted by the authors.

\section{Figure 7: Educational Attainment of Mother}

(\%)

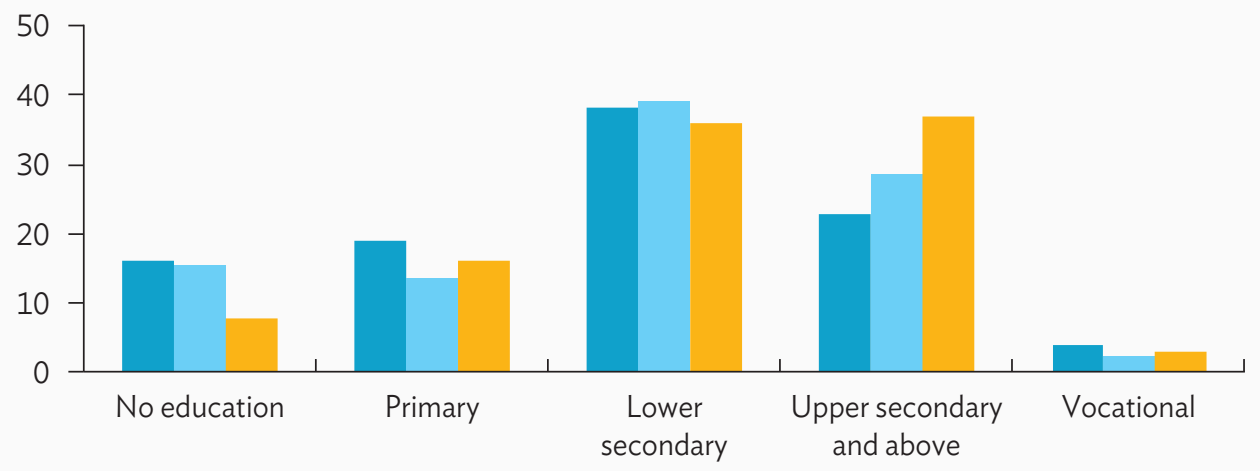

Source: Baseline survey conducted by the authors.

Figure 8: Educational Attainment of Father

(\%)

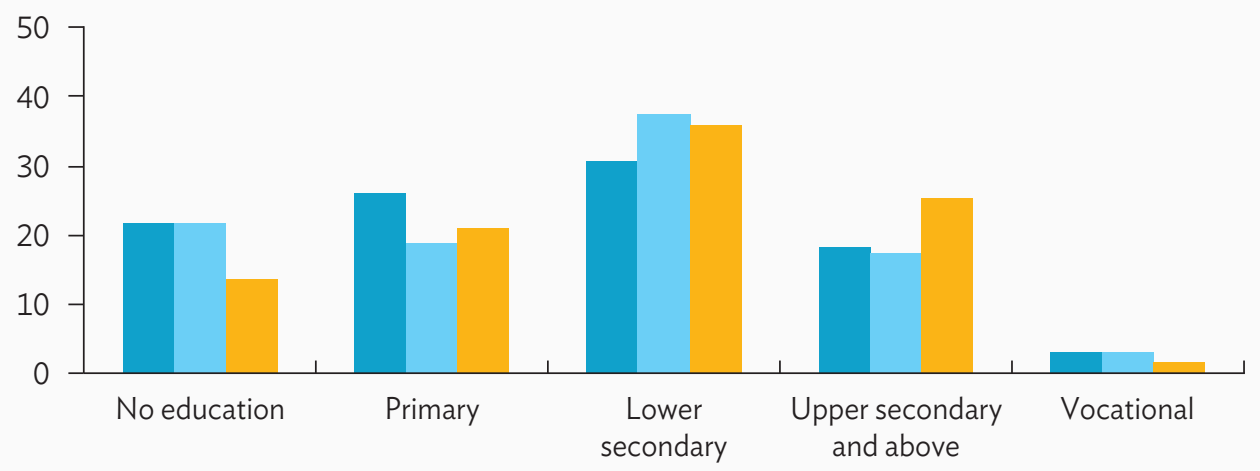

Source: Baseline survey conducted by the authors. 
Figure 9: Mother's Employment

(\%)

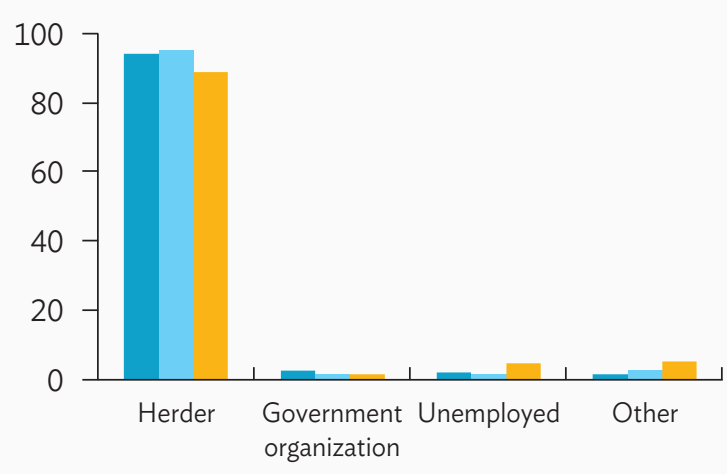

Full treatment

Light treatment

Comparison

Source: Baseline survey conducted by the authors.
Figure 10: Father's Employment

(\%)

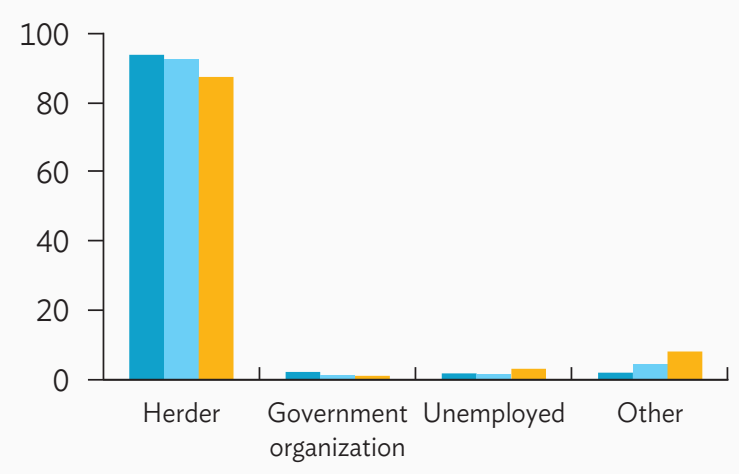

Full treatment

Light treatment

Comparison

Source: Baseline survey conducted by the authors.

Parents in the full treatment group (94\% of mothers and fathers) were herders, while in the light treatment group, herders accounted for $95 \%$ of mothers and $93 \%$ of fathers (Figures 9 and 10). The proportion of herders was slightly lower in the comparison group (89\% of mothers and $88 \%$ of fathers). Less than $5 \%$ of mothers and $3 \%$ of fathers were unemployed, with the proportion slightly higher in the comparison group.

On average, parents in the full treatment group had an annual income of MNT6,875,000 $(\$ 2,862)$, which was MNT673,000 (\$280) less than the average annual income of parents in the light treatment group, and MNT880,000 (\$366) less than that in the comparison group (Figure 11). ${ }^{10}$

The main income source for all the groups was sale of wool and cashmere, which constituted $55 \%$ of income for the full treatment group, 57\% for the light treatment group, and $43 \%$ for the comparison group (Figure 12). The second largest income source for the full treatment group was childcare subsidies (14\%), followed by sale of livestock or meat (11\%). By contrast, sale of livestock or meat was the second largest source of income for the light treatment (13\%) and comparison (20\%) groups, followed by childcare subsidies (12\%).

Overall, the figures indicate that students in the full treatment group came from poorer households, which depended slightly more on childcare subsidies and government allowance, and their parents were less educated than those in the light treatment and comparison groups.

10 The official exchange rate of the Central Bank of Mongolia on 29 April 2018 was used: $\$ 1=$ MNT2,401.65. 
Figure 11: Mean Income of Parents in the Last 12 Months

(MNT)

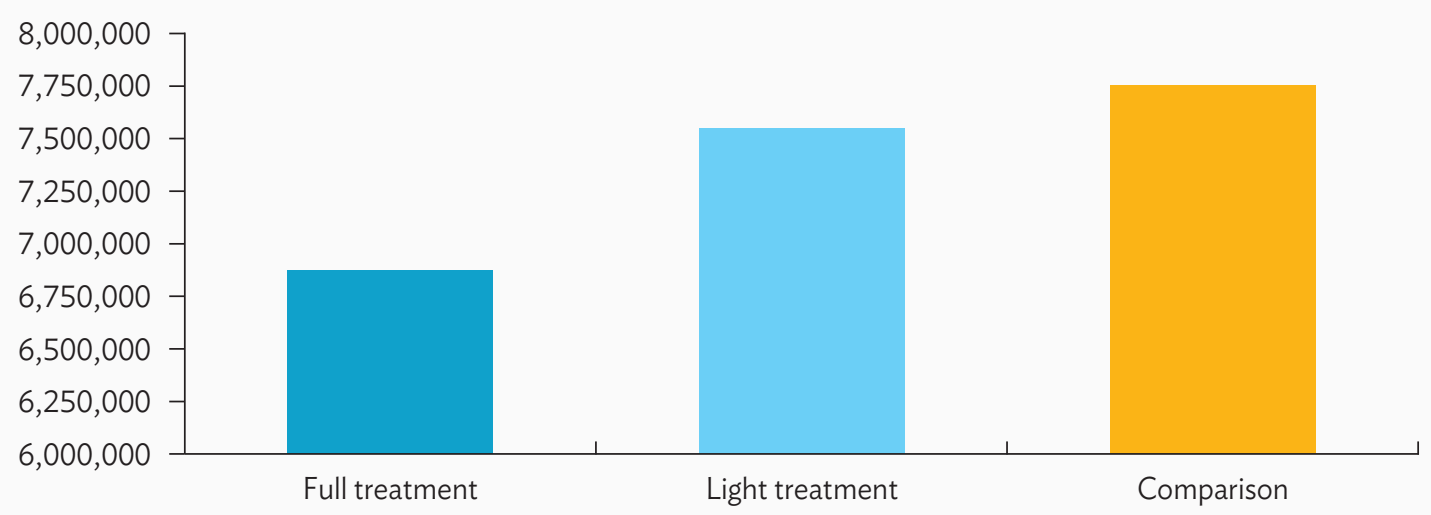

Source: Baseline survey conducted by the authors.

Figure 12: Income Sources of Parents

$(\%)$

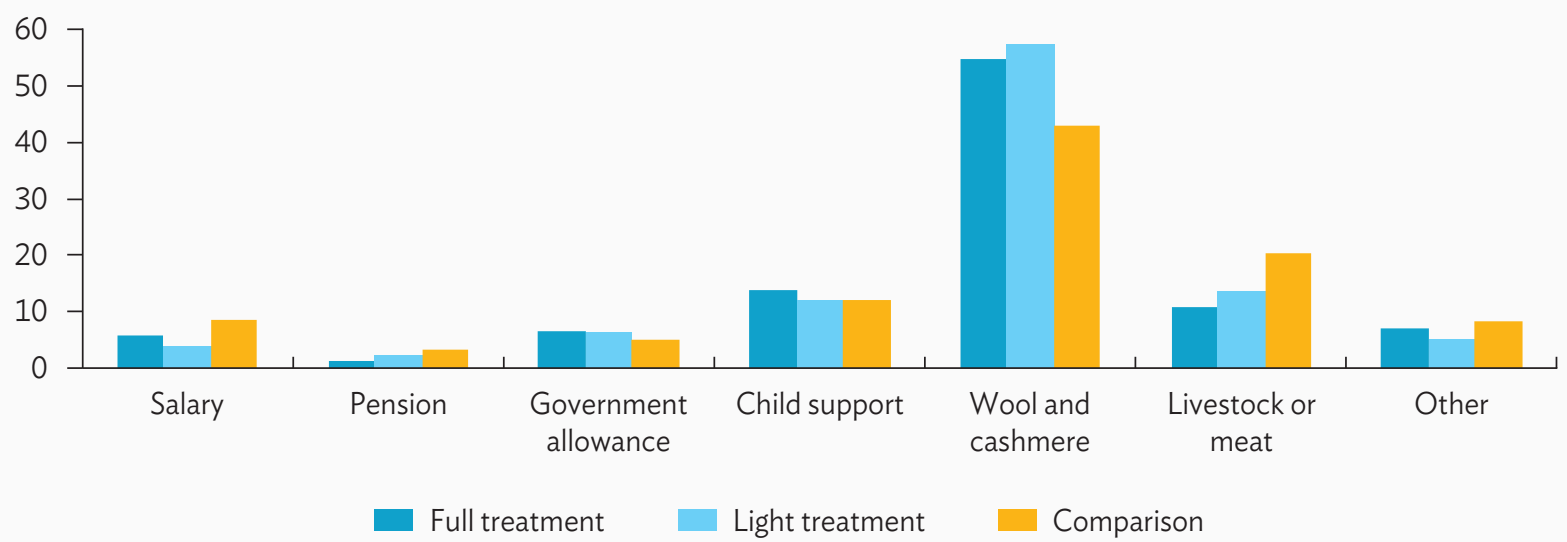

Source: Baseline survey conducted by the authors.

\section{B $\mid$ PHYSICAL DORMITORY ENVIRONMENT}

In the full treatment group, 24 dormitories were to be renovated under the project to (i) adequately cope with winter temperatures, wind, and precipitation; (ii) be equipped with WASH facilities to meet MECSS's minimum requirements, including those related to gender and early-grade students; (iii) accommodate larger numbers of students; and (iv) have common rooms for study, reading, and extracurricular activities. The renovation is expected to help students more safely and comfortably meet their basic physical needs, feel more at home, and better participate in study, reading, and extracurricular activities, which are in turn expected to improve their school attendance and learning outcomes. 


\section{(i) Dormitory Conditions}

Many of the dormitories in the survey were built in the 1970s and 1980s. The average year of construction for dormitories in the full treatment group was 1982 with more than half built before 1980 (Table 1).

For the light treatment group, the average year of construction was 1985, while for comparison dormitories, it was 1989 with half of these built before 1985. A more detailed overview of dormitories and their construction years is presented in Annex 5.

Table 1: Average Construction Year of Dormitories ${ }^{a}$

\begin{tabular}{l|c|c|c} 
& Full Treatment & Light Treatment & Comparison \\
\hline Year & 1982 & 1985 & 1989 \\
\hline Number & 29 & 53 & 81 \\
\hline
\end{tabular}

a The total number of dormitories in each of the three groups exceeds the number of dormitories included in the survey. This is because the information was provided by school principals who reported all dormitories belonging to the school, regardless of whether these were included or not in the survey.

Source: Baseline survey conducted by the authors.

Baseline data show that the majority of dormitories in all three groups-full treatment dormitories (72\%), and light treatment and comparison dormitories (77\%) - had been renovated at least once (Table 2). Of those dormitories that had undergone renovation, the average year of renovation was 2009 for the full treatment and comparison groups and 2010 for the light treatment group (Table 3).

Table 2: Dormitories with Previous Rehabilitation

\begin{tabular}{l|c|c|c} 
& Full Treatment & Light Treatment & Comparison \\
\hline Percentage & 72 & 77 & 77 \\
\hline Number & 21 & 41 & 62 \\
\hline
\end{tabular}

Source: Baseline survey conducted by the authors.

Table 3: Average Year of Last Rehabilitation ${ }^{a}$

\begin{tabular}{l|c|c|c} 
& Full Treatment & Light Treatment & Comparison \\
\hline Year & 2009 & 2010 & 2009 \\
\hline Number & 20 & 38 & 51 \\
\hline
\end{tabular}

a The number of dormitories in Table 2 and Table 3 differ because not all dormitories with reported previous rehabilitation were accompanied with a corresponding year.

Source: Baseline survey conducted by the authors. 
In sum, full treatment group of dormitories were, on average, constructed earlier than dormitories in the other groups. Also, fewer of these dormitories had undergone renovation, compared with those in the light treatment and comparison groups. As a result, dormitories often lacked adequate sanitation and hygiene facilities, heating systems, and protection from precipitation and wind (see photos in Annex 8). Baseline data show that students in the full treatment group were more likely to report feeling cold in winter (20\%), experience leaking dormitory ceilings (16\%), or wind blowing through dormitory windows (33\%) than those in the other groups. Although these problems were also reported by students in the light treatment and comparison groups, the proportion was lower by 5-10 percentage points (Figure 13).

\section{Figure 13: Students' Experiences in a Dormitory Environment}

$(\%)$

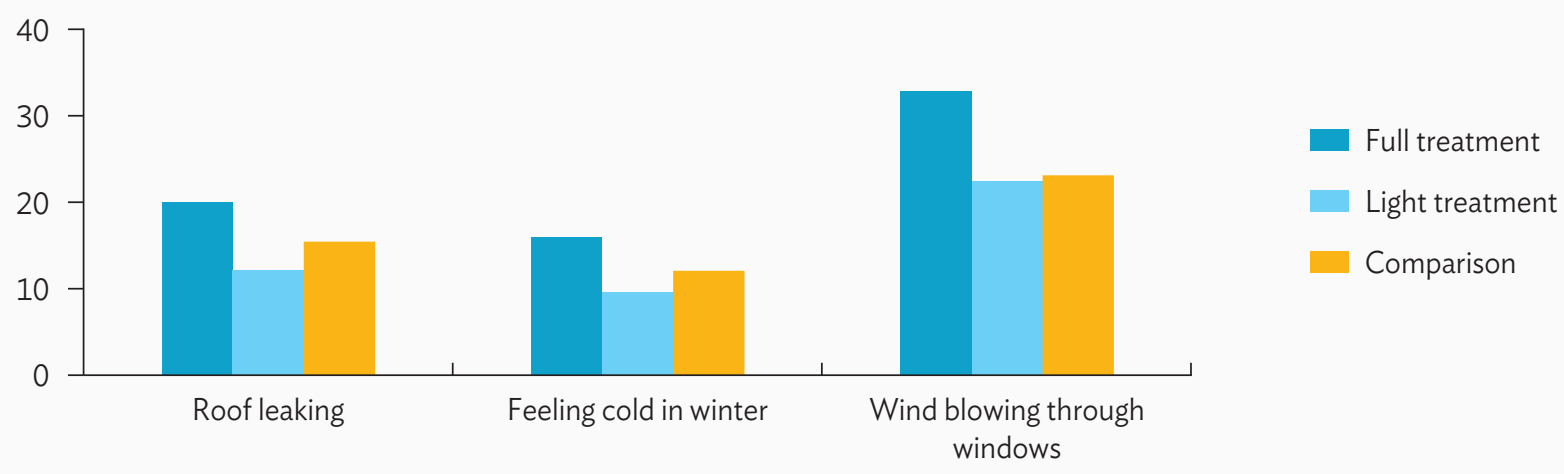

Source: Baseline survey conducted by the authors.

\section{(ii) WASH Facilities and Hygiene Practices}

According to the MECSS's minimum requirements for WASH facilities, indoor and outdoor toilets should be child-friendly, suitable for children's age, gender-sensitive (i.e., separate toilets for boys and girls), and suitable for children with disabilities (at least one toilet). ${ }^{11}$ In practice, many dormitories still do not meet these requirements. Among dormitories in all three groups, only about 37\% had indoor toilets, a little over $30 \%$ of these were rated suitable for early-grade students, and less than $10 \%$ were rated suitable for students with disabilities by enumerators (Figure 14). Two-thirds of dormitories had only outdoor latrines. Although many outdoor latrines were rated suitable for early-grade students by enumerators, the following outdoor latrines in dormitories were rated unsuitable: 35\% at full treatment group, $41 \%$ at light treatment group, and $26 \%$ at comparison dormitories (Figure 15). The latrines' suitability for students with disabilities was even poorer-only two units met the minimum requirements at full treatment group (8.3\%), six at light treatment group (11\%), and 20 at comparison dormitories (25\%).

11 MECSS, Ministry of Health, and Ministry of Finance. 2015. Joint Ministerial Order on "Norms and Requirements of Water, Sanitation, and Hygiene for Kindergartens, Secondary Schools and Dormitories." Ulaanbaatar. 


\section{Figure 14: Latrine Availability and Suitability for Early Graders and for Children with Disabilities}

(\%)

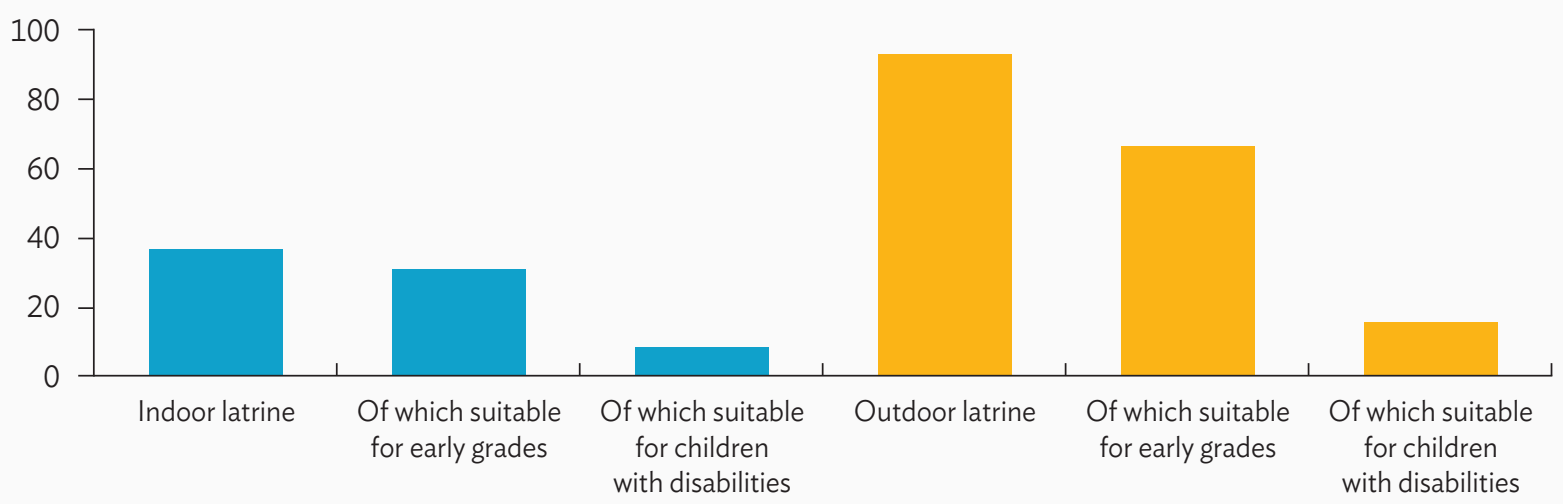

Source: Baseline survey conducted by the authors.

Figure 15: Dormitories with Outdoor Latrine and Suitability to Vulnerable Groups

$(\%)$

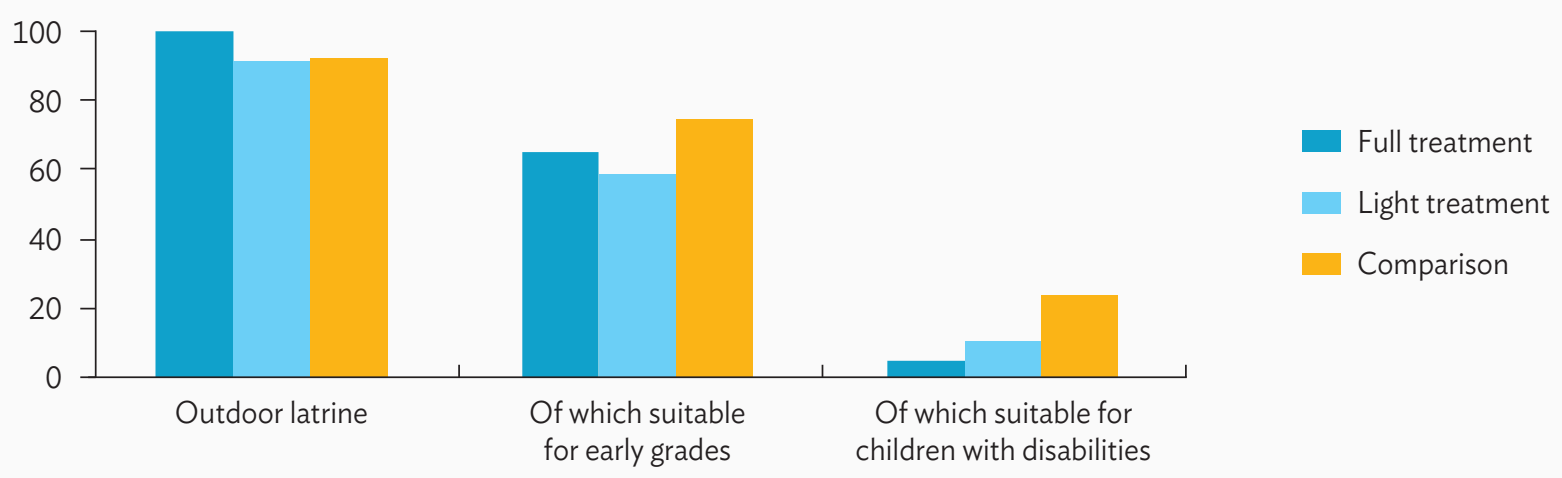

Source: Baseline survey conducted by the authors.

Although latrines for girls and boys were separated by walls in about $90 \%$ of dormitories, they had individual doors only in around $60 \%$ of dormitories, and locks in even less than $30 \%$ of dormitories (Figure 16).

Latrines with locks were available only in three dormitories of the full treatment group (12.5\%), compared to $25 \%$ in the light treatment group, and $29 \%$ in comparison dormitories. The availability of hygiene bins was also limited (39\% in comparison dormitories, the highest among all three groups). Overall, the condition of toilets for girls were worst at dormitories in the full treatment group, slightly better in the light treatment group, and the best in comparison dormitories. 


\section{Figure 16: Toilet Facilities for Girls}

(\%)

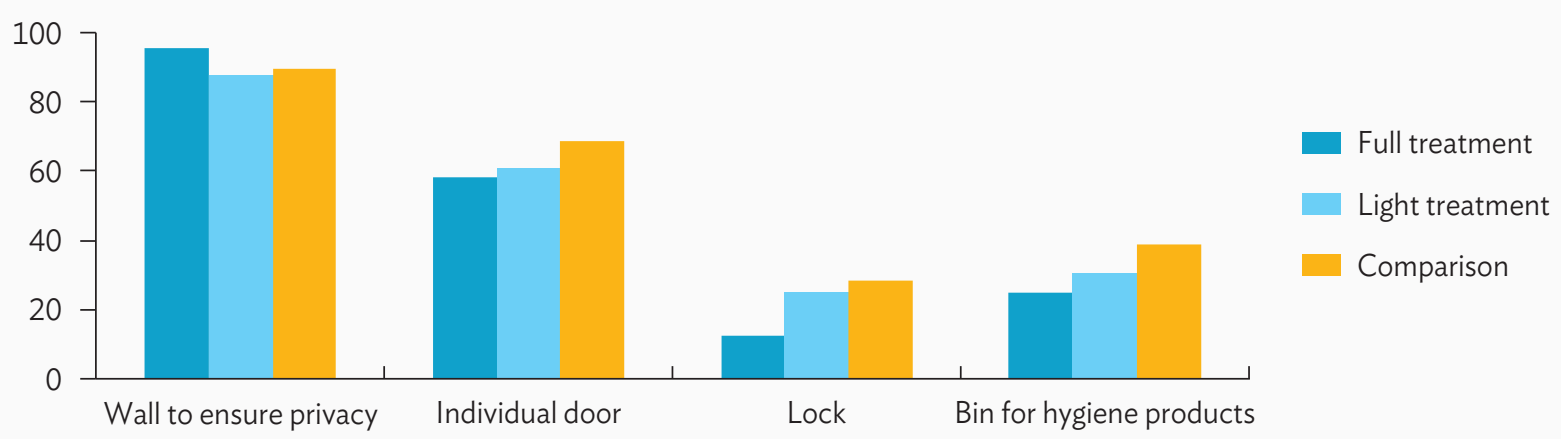

Source: Baseline survey conducted by the authors.

Since most dormitories did not have indoor toilet facilities, the majority of resident students, regardless of their ages, had to venture outside to go to the toilet at night. Overall, about $40 \%$ of students reported being afraid of going to the toilet at night, with the largest proportion among students in early grades, and a slightly higher proportion in the full treatment group than in the light treatment and comparison groups (Figure 17).

Moreover, no tap water was available in most dormitories, which could affect the students' hygiene practices. The most frequent hygiene practice among the students was brushing their teeth in the morning, followed by handwashing before eating (Figure 18). While 33\% of students responded that they always wash hands after going to the toilet, $40 \%$ answered that they never or hardly ever do so. Similarly, while 38\% responded that they always shower once a week at the dormitory, $41 \%$ answered that they never or hardly ever do so.

Figure 17: Fear of Going to the Toilet at Night, by Grade

$(\%)$

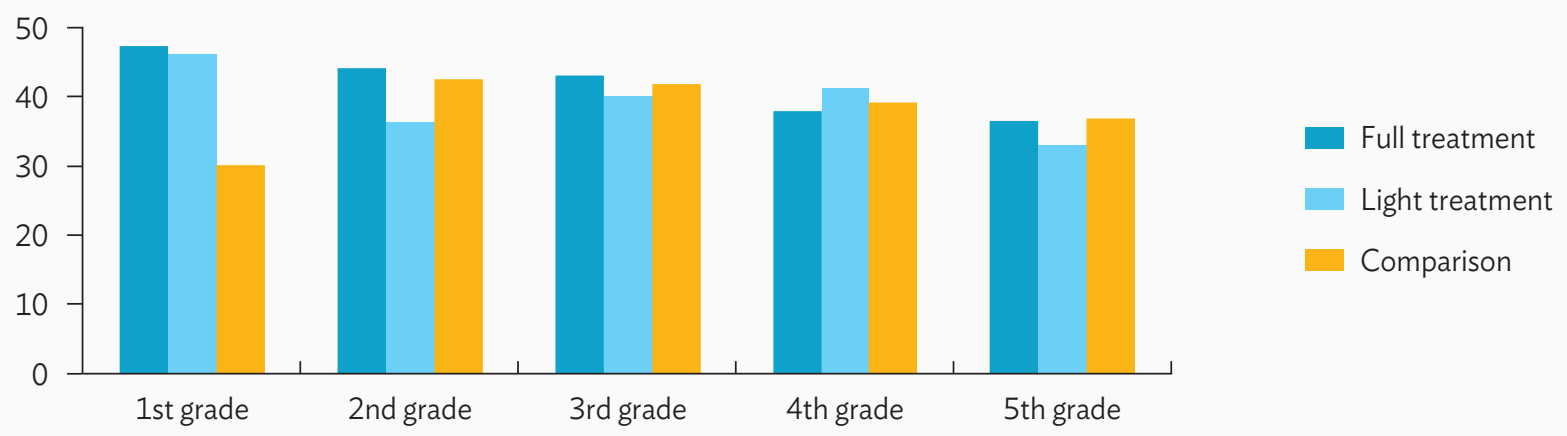

Source: Baseline survey conducted by the authors. 


\section{Figure 18: Hygiene Practices of Dormitory Students}

$(\%)$

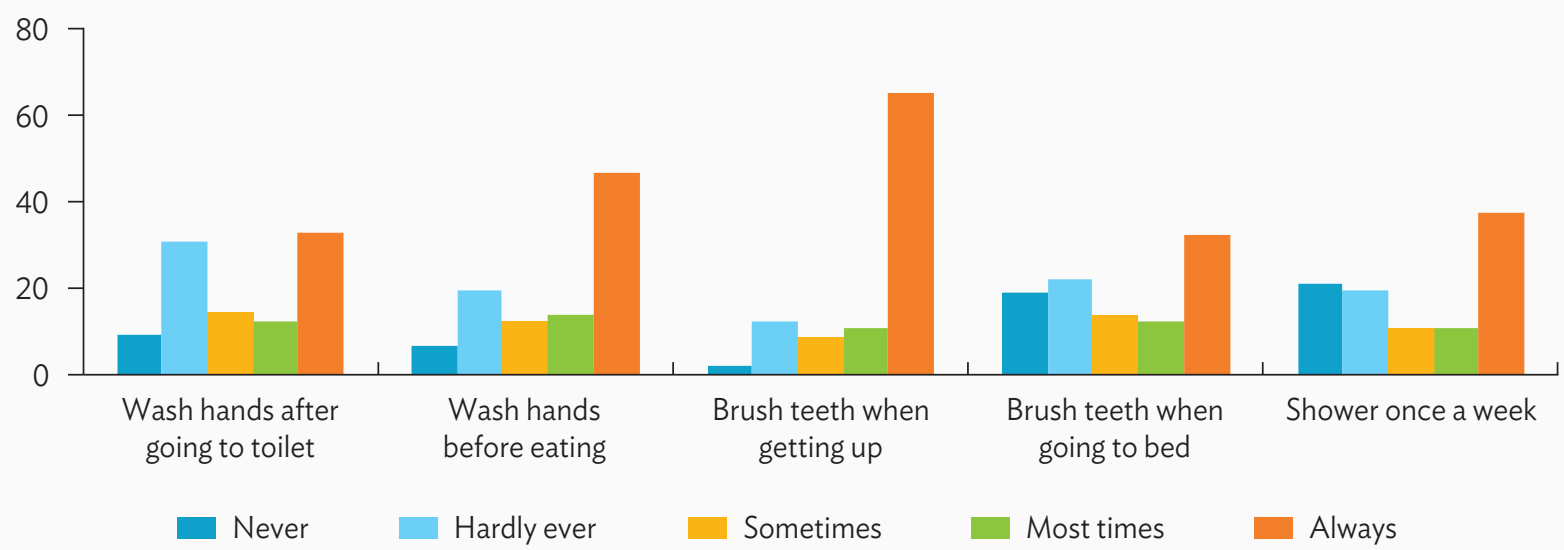

Source: Baseline survey conducted by the authors.

The frequency of handwashing after going to the toilet and taking a shower once a week was very similar among the students across all three groups (Figures 19 and 20).

Figure 19: Student Hygiene Behavior:

Frequency of Washing Hands after Going to the Toilet

(\%)

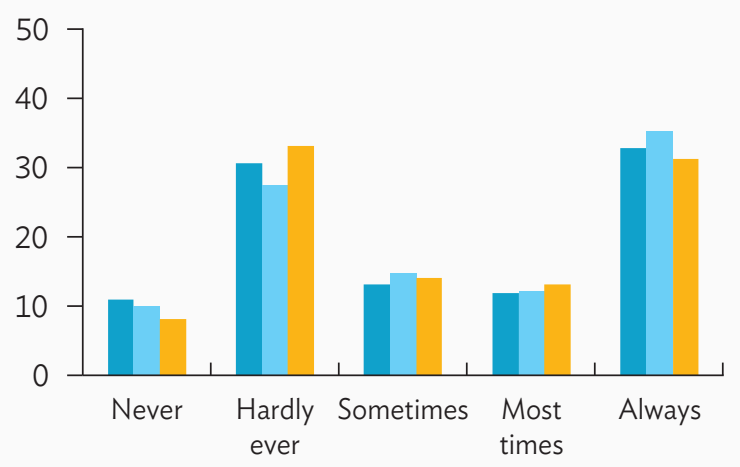

Full treatment

Light treatment

Comparison

Source: Baseline survey conducted by the authors.
Figure 20: Student Hygiene Behavior:

Taking a Shower Once a Week at Dormitory

(\%)

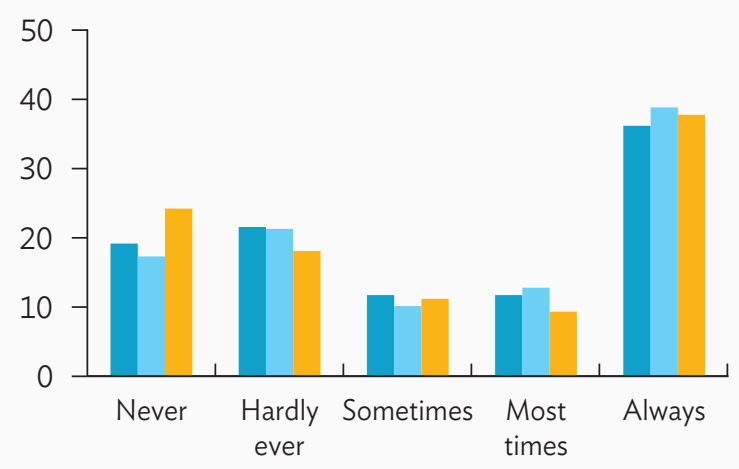

Full treatment

Light treatment

Comparison

Source: Baseline survey conducted by the authors. 


\section{(iii) Furniture and Common Rooms}

Enumerators observed that 70\%-79\% of dormitories had a sufficient number of beds (Figure 21). Where beds were insufficient, students needed to share a bed. Dormitories with sufficient number of chairs and tables were less common, with only $16 \%-26 \%$ of dormitories having an adequate number of chairs, and $20 \%-29 \%$ having enough tables.

\section{Figure 21: Availability of Dormitory Furnitures Based on Enumerator's Observation}

$(\%)$

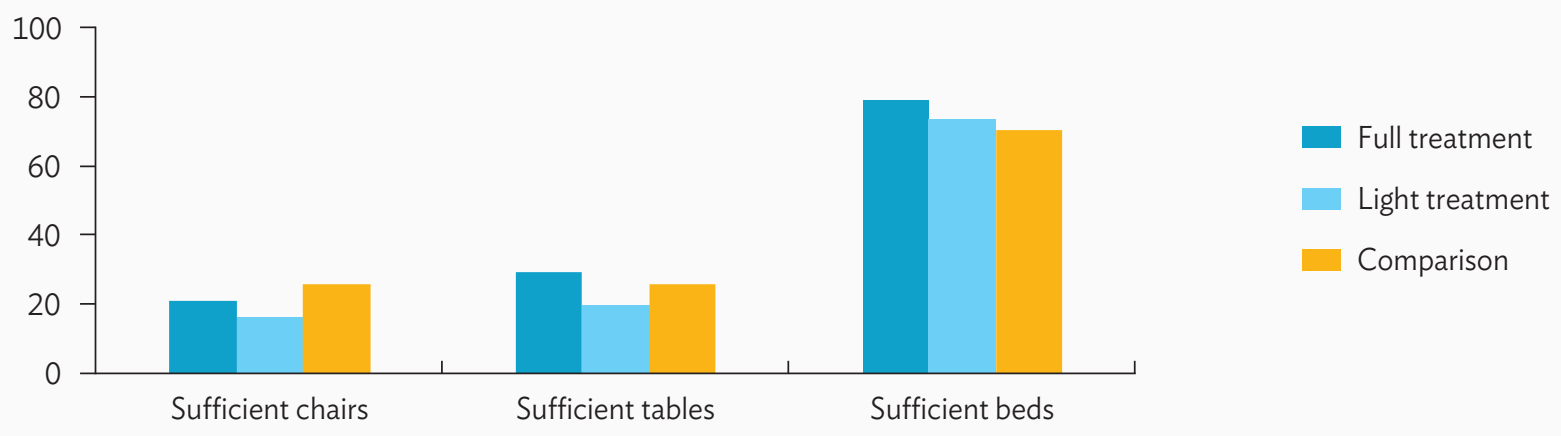

Source: Baseline survey conducted by the authors.

Of the dormitories in the full treatment and light treatment groups, $65 \%-70 \%$ had common rooms, while only a little over $50 \%$ of the comparison dormitories have them (Figure 22). Study rooms and libraries were found in $20 \%-35 \%$ of dormitories, while meeting rooms were rare in all the three groups.

\section{Figure 22: Dormitory Facilities}

$(\%)$

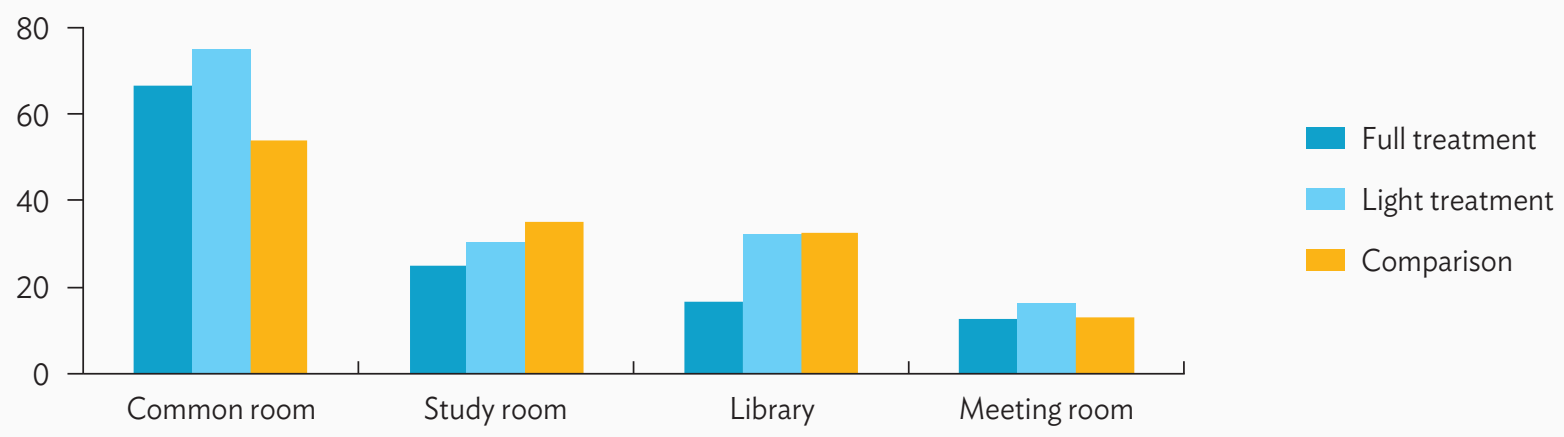

Source: Baseline survey conducted by the authors. 
Figure 23: Common Room with Equipment

(\%)

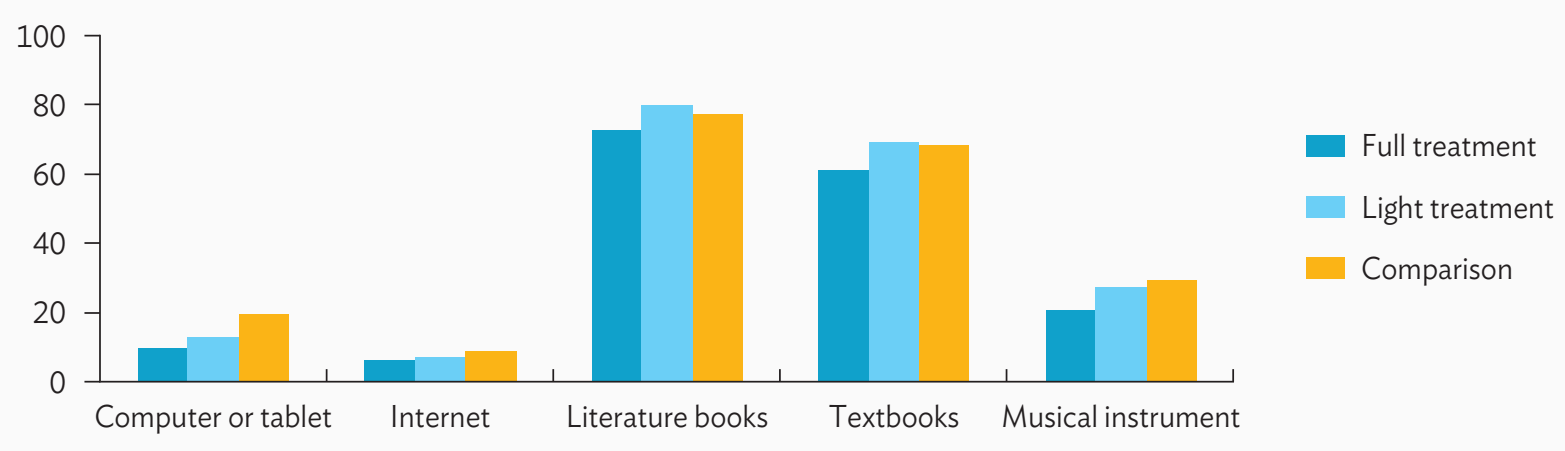

Source: Baseline survey conducted by the authors.

Over $70 \%$ of the common rooms had literature books and slightly fewer had textbooks (Figure 23). Musical instruments were found in $20 \%-30 \%$ of the common rooms. Less than $20 \%$ had computers and internet with a slightly higher proportion found in the comparison group.

Almost all students reported having a desk in which to study. Having a quiet place to study was only at $43 \%$ of students in the full treatment group while this is $56 \%$ in the light treatment and comparison groups (Figure 24). The responses correspond well with the question of what disturbed them when they wanted to study. Although 44\% of students in the full treatment group and $47 \%$ in the light treatment group could not think of anything that disturbed them, more than half reported "other students" (Figure 25).

Finally, cooks were asked if kitchens were properly equipped with fire safety measures. Fire extinguishers of any type were available at only 2 out of 21 (9.5\%) in the full treatment group, compared to $44 \%$ in the light treatment group, and $59 \%$ in the comparison dormitories (Figure 26). ${ }^{12}$

12 The survey distinguished between fire hydrant, fire extinguishing automatic system, fire pipe that is connected to a central water system, or a traditional fire extinguisher.
Figure 24: Study Space in the Dormitory

$(\%)$

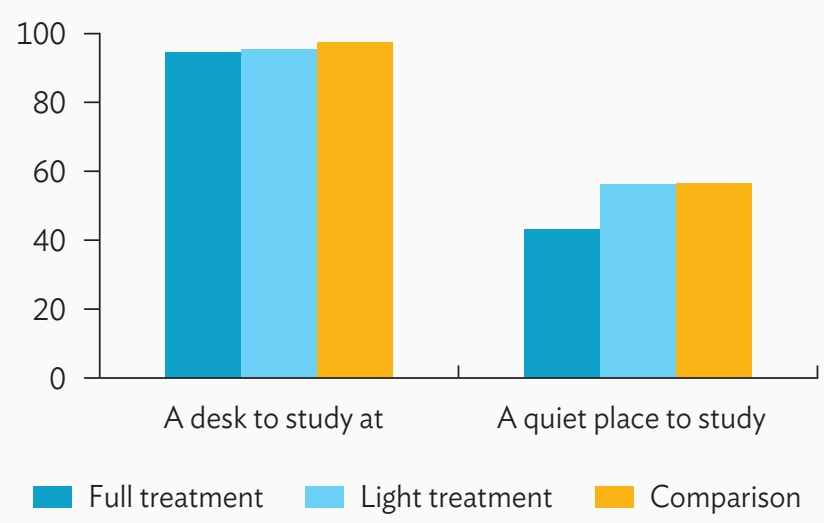

Source: Baseline survey conducted by the authors. 


\section{Figure 25: Students' Response to "What bothers you when you want to do your homework, study, read, and concentrate?"}

$(\%)$

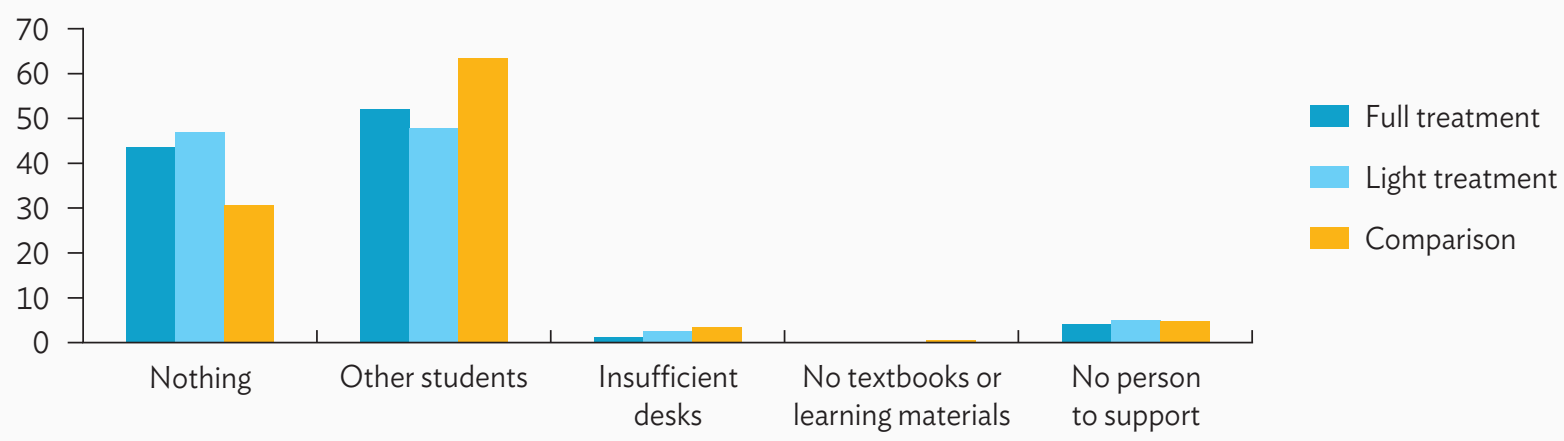

Source: Baseline survey conducted by the authors.

Figure 26: Presence of Any Kind of Fire Extinguisher in the Dormitory

$(\%)$

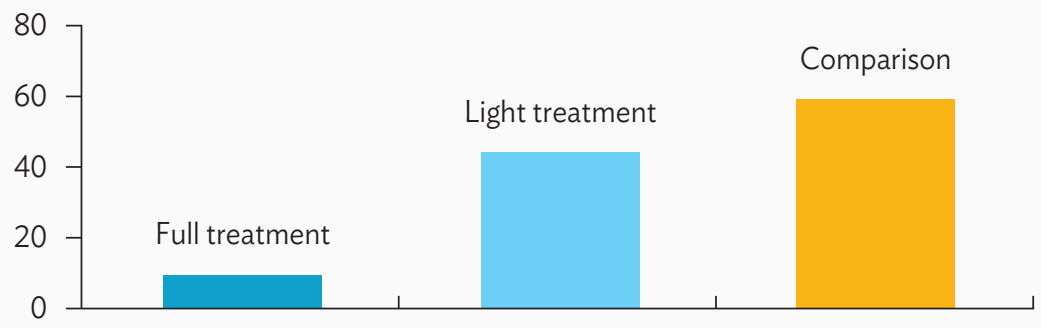

Source: Baseline survey conducted by the authors.

\section{C | CAPACITY TO DELIVER DORMITORY SERVICES}

Under the project, 78 dormitories in the full treatment and light treatment groups were to have their capacities enhanced to enable them to deliver improved dormitory services. These were to be done through the following trainings: (i) for dormitory teachers-on child abuse issues and child-centered methods to facilitate study, reading, and extracurricular activities, and improve the school preparedness of early-grade students; (ii) for dormitory guards-on child abuse issues and child-centered communication skills to encourage study, reading, and extracurricular activities, and to take care of students at night, especially those in early grades; (iii) for school staff involved in preparing dormitory meals - on the nutritional requirements of students; (iv) for school management, accountants, and aimag education administration staff-on the use of tools and reference materials to enhance the management of financial, human, and physical resources for school dormitories; and (v) for dormitory teachers, primary class teachers, and social workers - on student- and teacher-organized study, reading, and extracurricular activities at dormitories, and communication and outreach activities for the parents. 
The enhanced capacity is expected to help the students feel more supported, understood, and at home; avoid getting sick, be healthier and more active; and become more motivated to participate in study, reading, and extracurricular activities, which are in turn expected to improve their school attendance and learning outcomes.

\section{(i) Previous Trainings of Dormitory Staff}

Baseline data show that a substantial proportion of dormitory teachers reported having received training on child violence and conflict resolution (over $50 \%$ in both full treatment and light treatment groups), while about one-third had received training on child-centered methodology (Figure 27). The proportion of teachers at comparison dormitories who received these two types of training was smaller.

Figure 27: Dormitory Teachers Who Received Training, by Topic

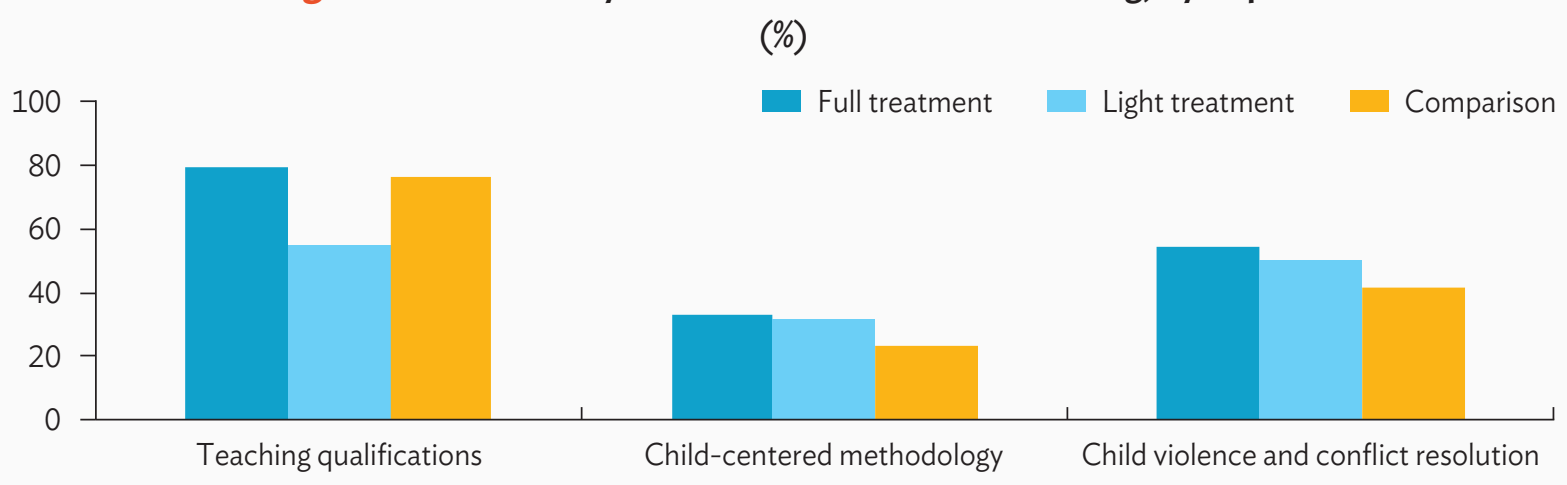

Source: Baseline survey conducted by the authors.

By contrast, the proportion of dormitory guards who underwent any kind of training was smaller across all the groups (Figure 28). The most frequent kind of training was on child violence and conflict resolutionwhich more than 25\% of guards (full treatment and light treatment groups) had attended, compared to 39\% of guards in the comparison dormitories. Less than $20 \%$ of guards (19\% at full treatment group, $15 \%$ at light treatment group, and 13\% at comparison dormitories) underwent training on child-centered communication.

The proportion of dormitory cooks who were trained on any topic was larger than dormitory guards. The largest was in the light treatment group where $40 \%$ attended training on meal preparation, 58\% on food nutritional value, and $46 \%$ on food safety (Figure 29). In the full treatment group, the share was smaller-19\% on meal preparation, and $29 \%$ on food nutritional value and food safety.

\section{(ii) Outreach and Communication Activities for Parents}

Baseline data show that the proportion of teachers who reported having a plan to work with parents differed substantially between the groups. In the full treatment group, $46 \%$ of teachers responded positively, $68 \%$ in the light treatment group, and 62\% in the comparison group (Figure 30). 
Figure 28: Dormitory Guards Who Received Training, by Topic

(\%)

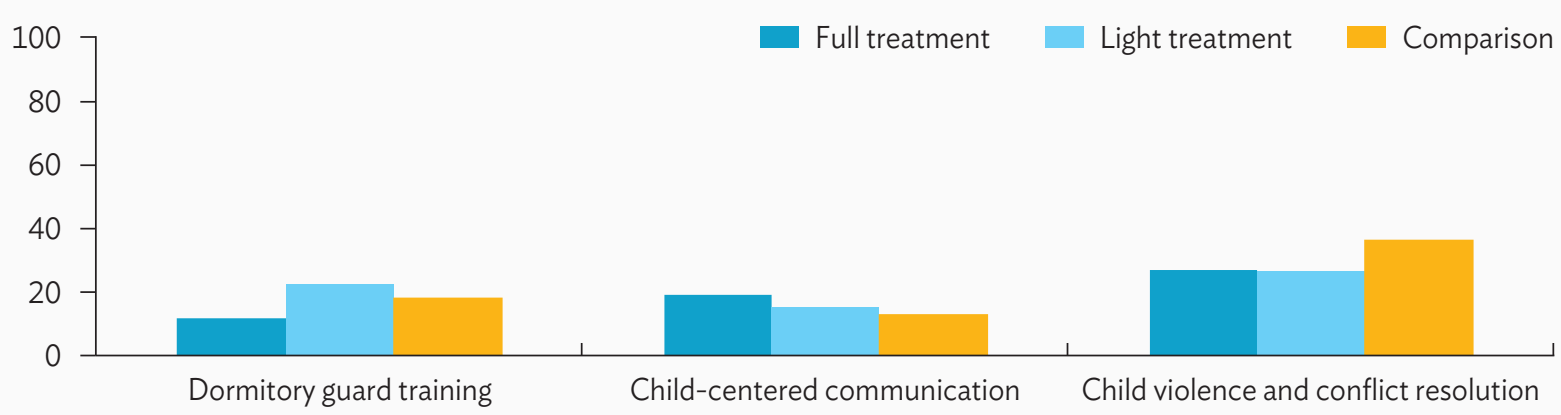

Source: Baseline survey conducted by the authors.

Figure 29: Cooks Who Received Training, by Topic

$(\%)$

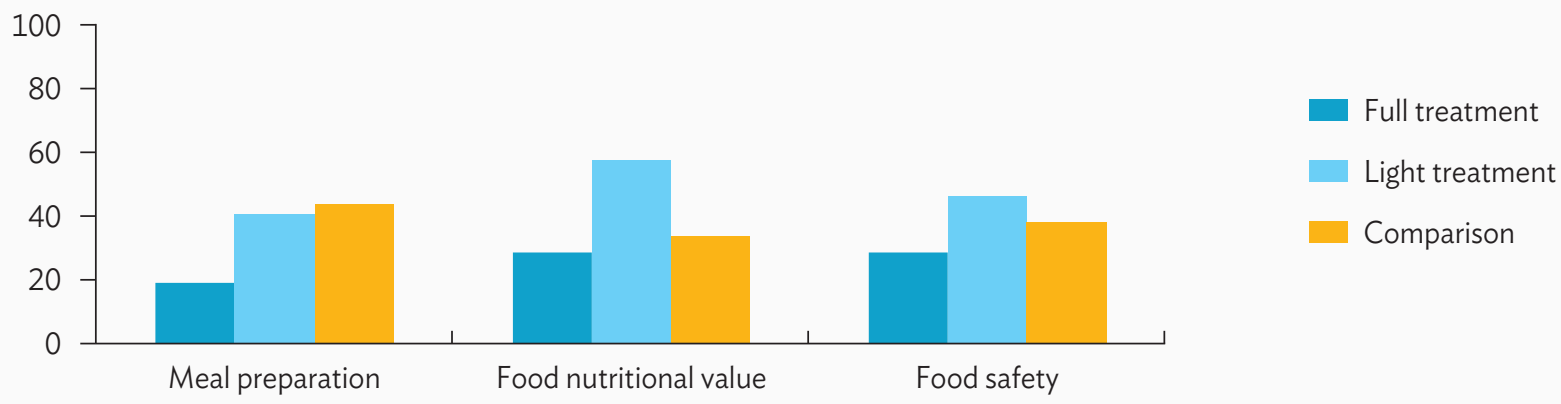

Source: Baseline survey conducted by the authors.

Figure 30: Teachers Reporting Having a Plan to Work with Parents, by Group

$(\%)$

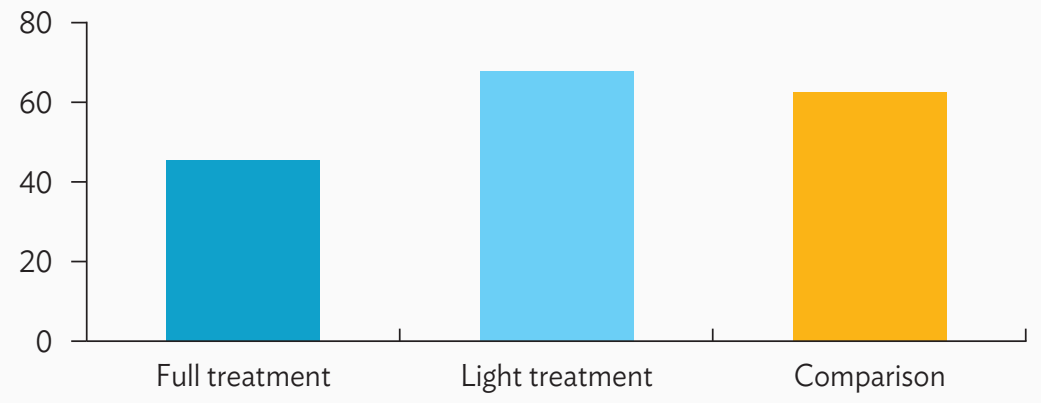

Source: Baseline survey conducted by the authors. 


\section{Figure 31: Parents' Observation of School-Organized Activities}

(\%)

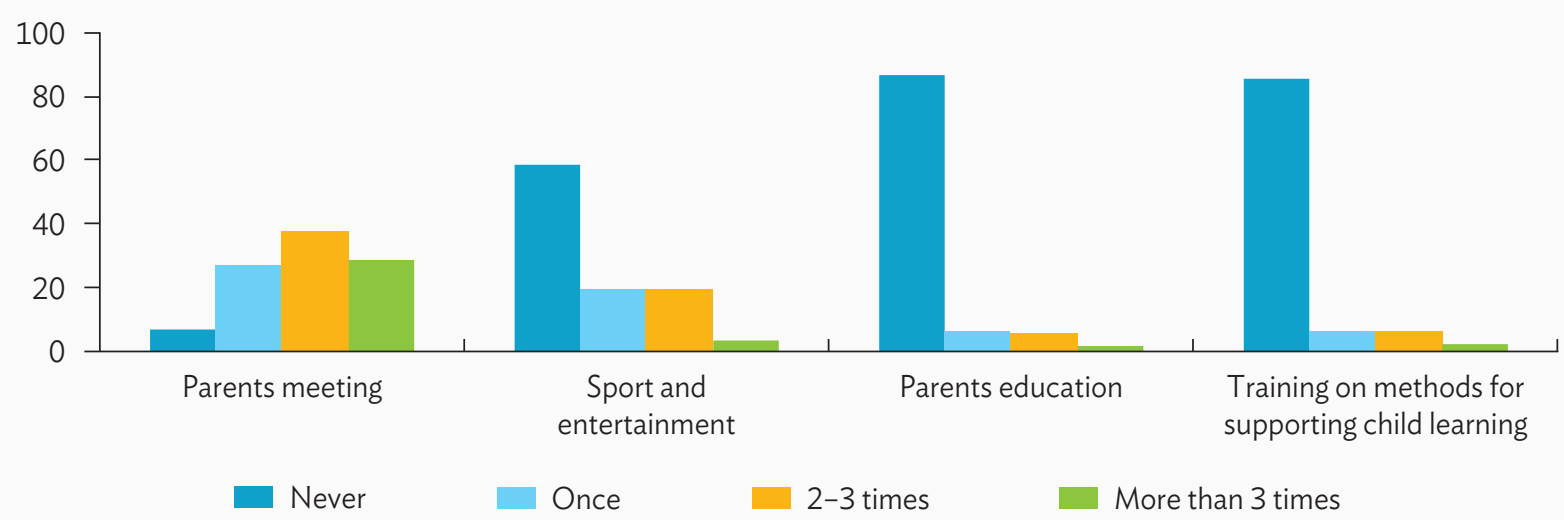

Source: Baseline survey conducted by the authors.

Few parents reported being aware of any school-organized education or training activities for parents during SY2016/17. The majority also rarely heard about sports and entertainment activities, except for some $20 \%$ who had been aware of these in more than 2-3 occasions (Figure 31). Parents' meetings were more popular, with $93 \%$ of parents having heard of such meeting at least once. Although close to $95 \%$ of parents responded being aware of at least one parents' meeting, about half of the parents did not attend any during SY2016/17 (Figure 32). Those who participated in school-organized sports and entertainment activities were smaller-16\% in both full treatment and light treatment groups, and 19\% in the comparison group. Less than $10 \%$ of the parents attended education activities intended for parents.

\section{Figure 32: Parents' Participation in School-Organized Activities}

(\%)

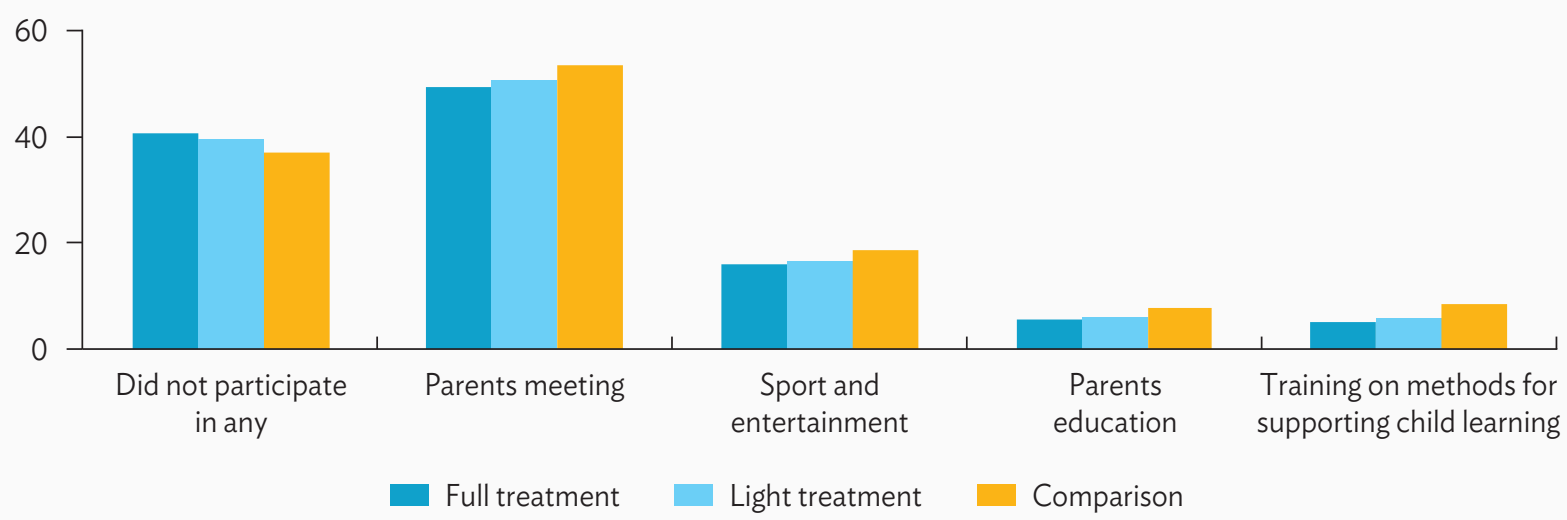

Source: Baseline survey conducted by the authors. 


\section{(iii) Student Committee and After-School Activities}

Baseline data show that almost all dormitories had student committees (Figure 33). Nevertheless, only 39\% in the light treatment group, $42 \%$ in the full treatment group, and $44 \%$ in the comparison group reported that teachers were organizing learning activities jointly with student committees.

\section{Figure 33: Dormitory Committees and Activity}

$(\%)$

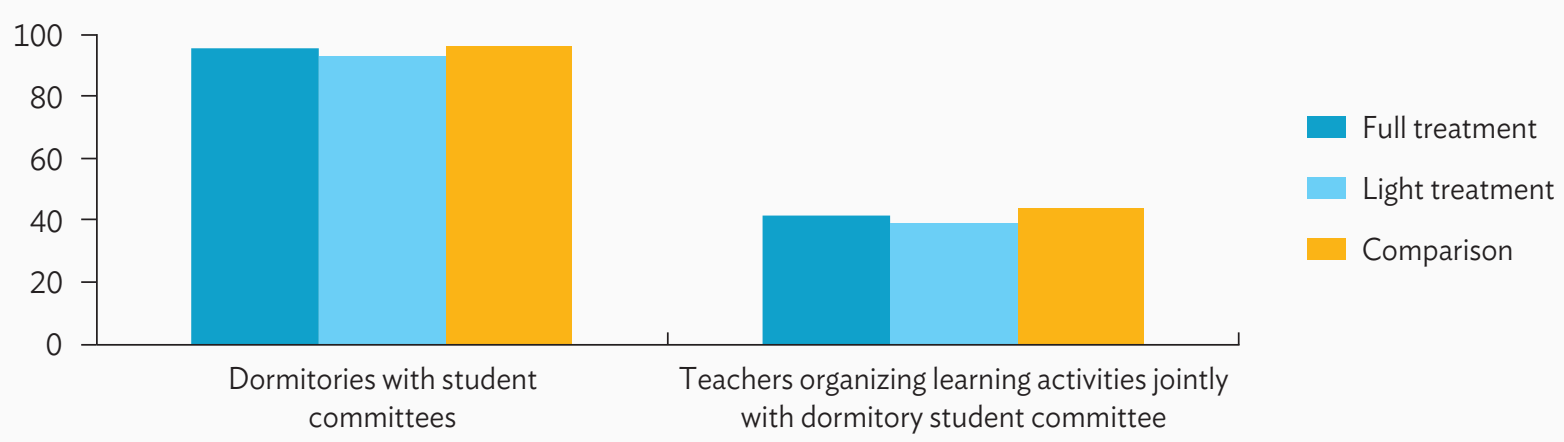

Source: Baseline survey conducted by the authors.

Student participation in after-school activities was similar across the three groups. About $40 \%$ of the girls took part in activities or clubs, while a slightly smaller proportion of boys did so (Figure 34). Among students residing in dormitories who did not participate in after-school activities or clubs, about $40 \%$ said they were not interested (Figure 35). The second common reason for not participating was the unavailability of such activities, as reported by $16 \%-19 \%$ of the students. Having no friends to go with, no money, or no time were given as reasons by $7 \%-15 \%$ of students.

Figure 34: Students Engaging in After-School Activities or Clubs

$(\%)$

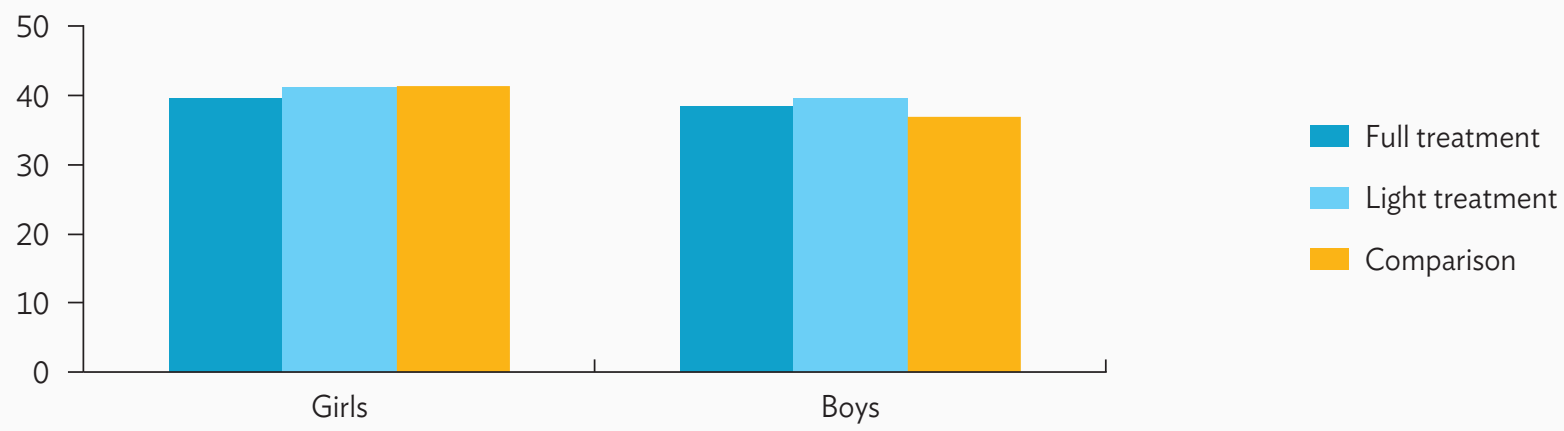

Source: Baseline survey conducted by the authors. 


\section{Figure 35: Reasons for Not Attending After-School Activities}

(\%)

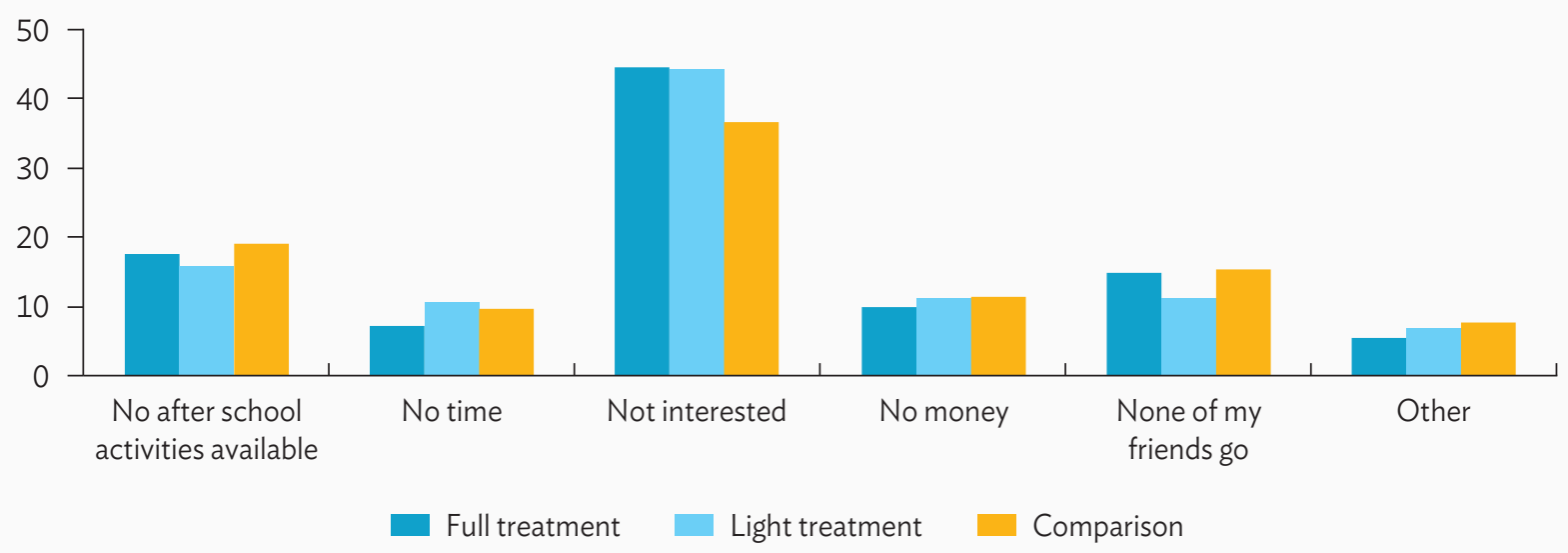

Source: Baseline survey conducted by the authors.

\section{(iv) Dormitory Meals}

Dormitories in the full treatment and light treatment groups served an average of 4.3 meals daily, including tea and snacks, as compared to the 4.1 times in the comparison group (Figure 36). The average number of daily ingredients used for dormitory meals was also similar across the three groups -6.3 in the full treatment group, 6.1 in the light treatment group, and 6.2 in the comparison group (Figure 37).

Figure 36: Average Number of Meals Served Daily

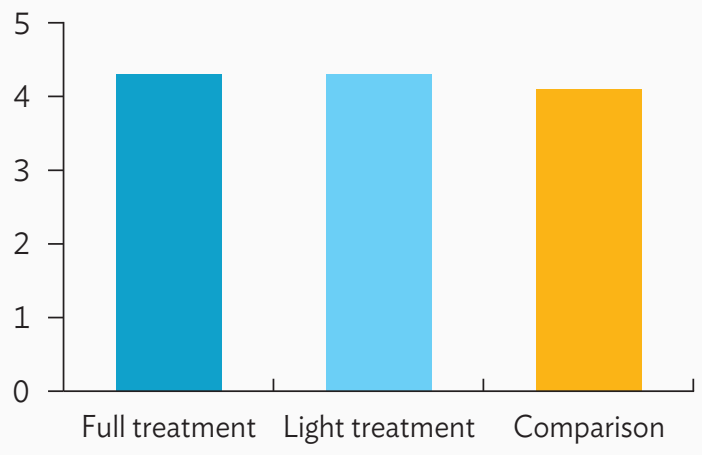

Source: Baseline survey conducted by the authors.
Figure 37: Average Number of Daily Ingredients

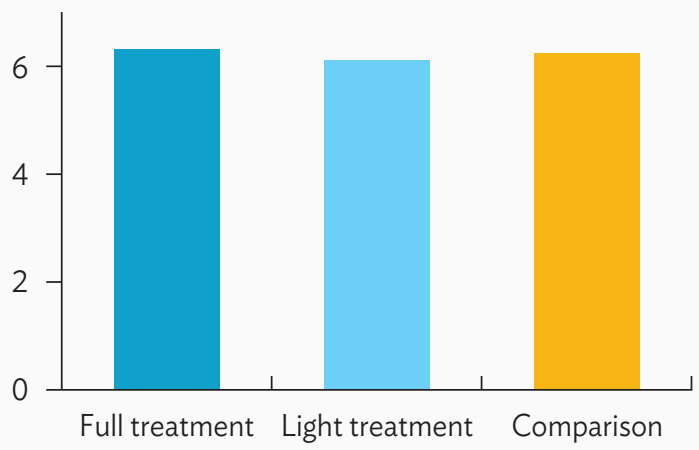

Source: Baseline survey conducted by the authors. 
Potato, onion, and carrot were the top three ingredients used in dormitory meals, almost daily. Milk was used 17 times a month on average (more than every other day), yoghurt or curd was served 6 times per month on average, and other dairy products were served 4 times per month on average (Figure 38 ). ${ }^{13}$

\section{Figure 38: Cooking Ingredients by Frequency of Use}

(average number of times used per month)

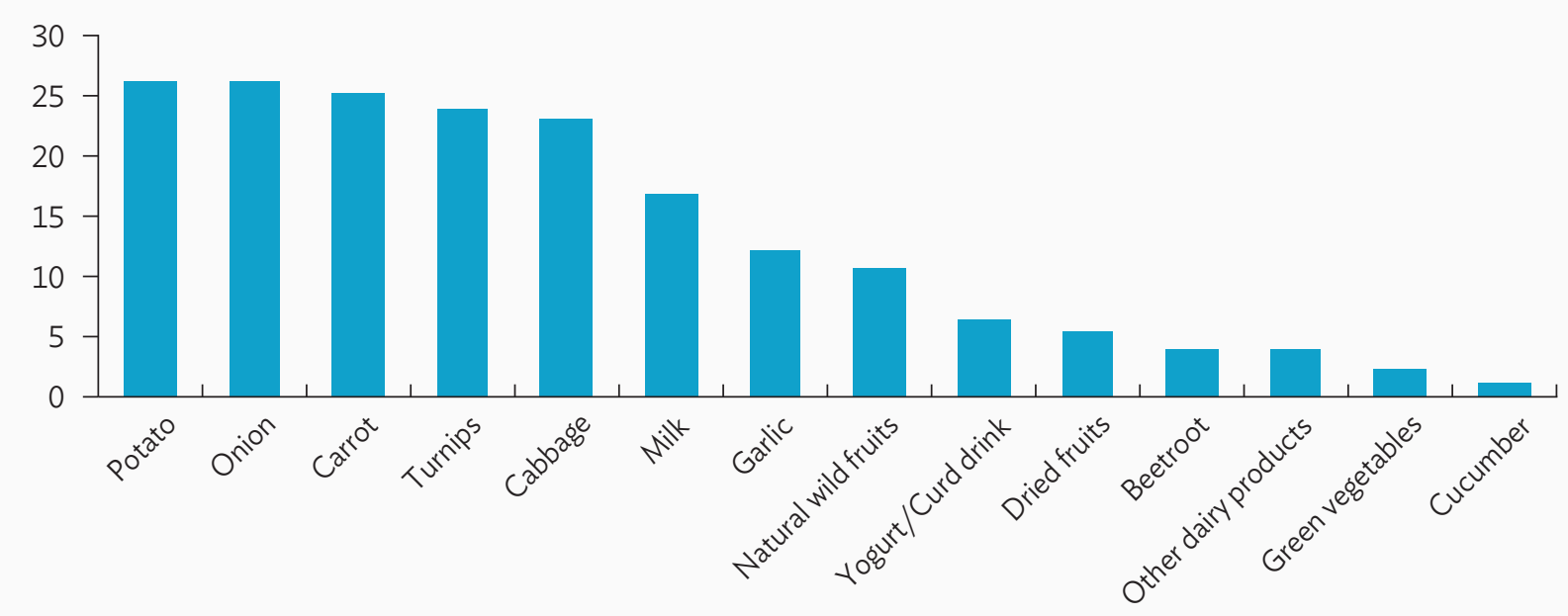

Source: Baseline survey conducted by the authors.

\section{(v) Parents' Involvement and Support}

When asked about the level of support coming from their parents, nearly $90 \%$ of the students reported feeling supported (Figure 39).

A greater proportion ( $94 \%$ in the full treatment and comparison groups, and $96 \%$ in the light treatment group) agreed or strongly agreed that their parents support their educational efforts and achievements (Figure 40).

Close to $90 \%$ of students across the three groups responded that their parents support them when they face physical or emotional difficulties at school (Figure 41).
Figure 39: My Parents Are Interested in My School Activities

(\%)

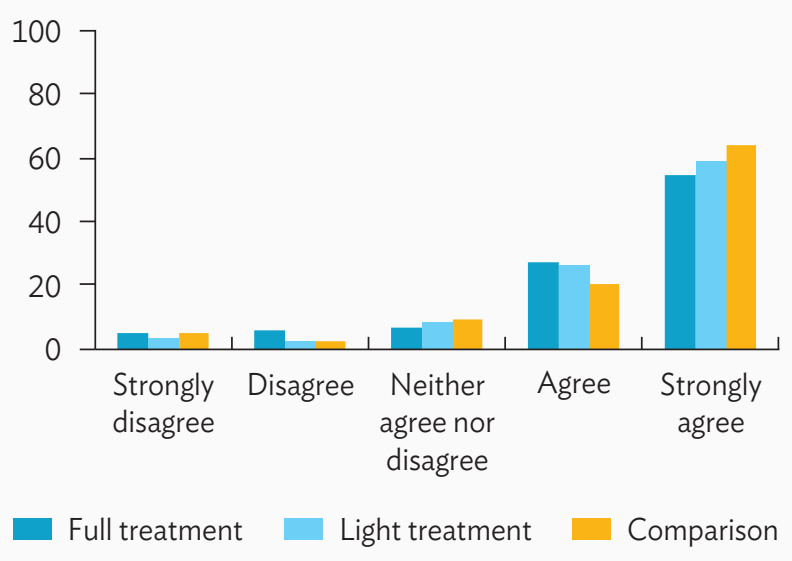

Source: Baseline survey conducted by the authors.

13 Meat was not included as it was expected to be a regular and/or daily ingredient. 
Figure 40: My Parents Support My Educational Efforts and Achievements

(\%)

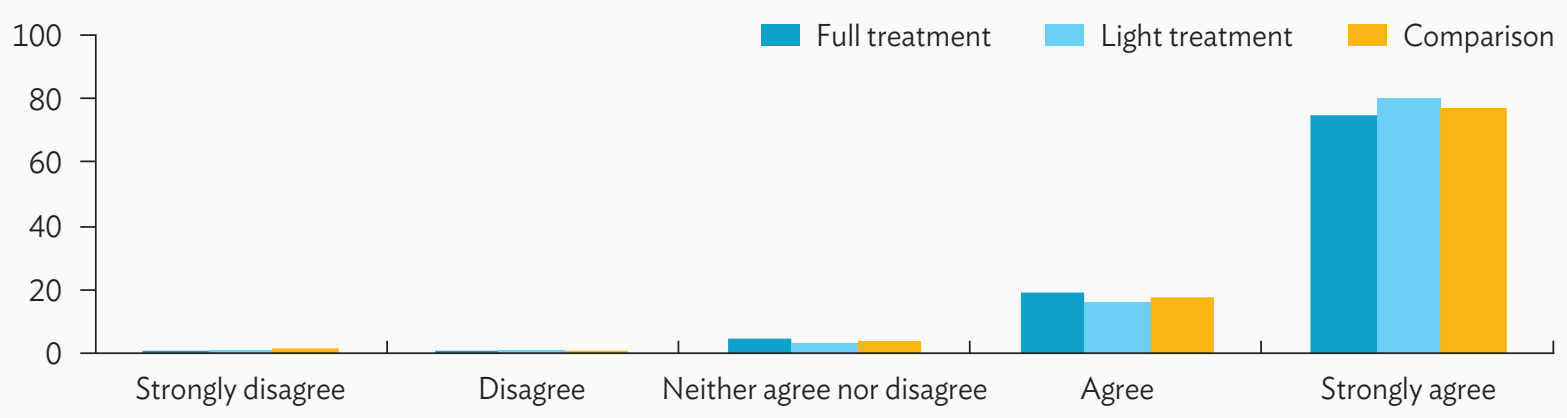

Source: Baseline survey conducted by the authors.

Figure 41: My Parents Support Me When I Am Facing Difficulties at School

(\%)

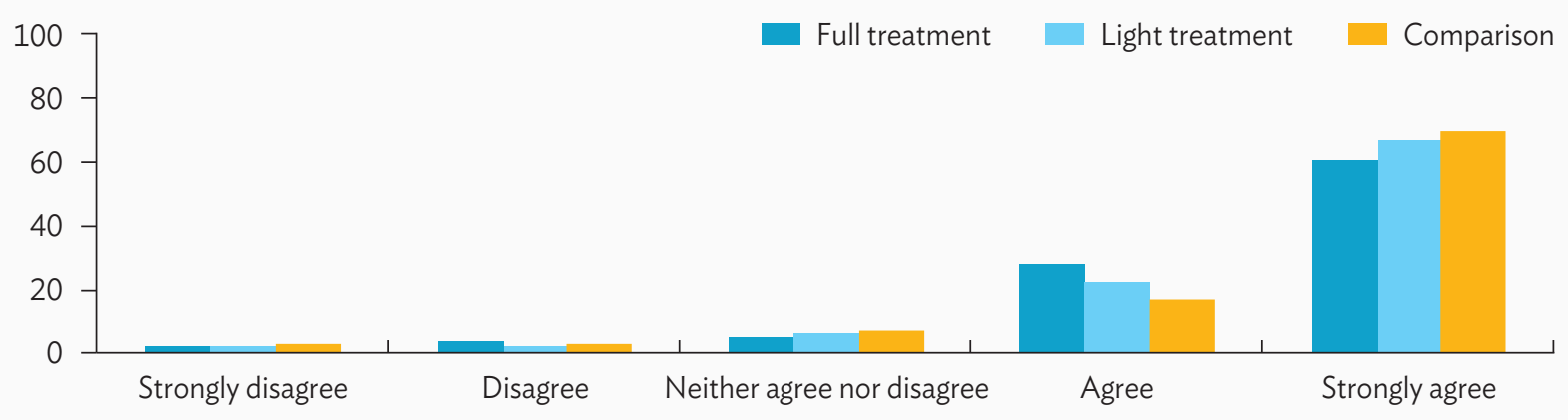

Source: Baseline survey conducted by the authors.

Parents also reported providing regular support to their children. Parents in the full treatment group spent 3.3 hours on average per week supporting their child, parents in the light treatment group spent 3.5 hours, while the comparison group spent 3 hours (Figure 42).

Since nearly $90 \%$ of students felt supported by their parents at baseline, efforts to increase parental support may have limited impact on student outcomes. Nevertheless, it must be noted that a non-negligible proportion of
Figure 42: Average Weekly Hours of Parental Support to Their Children

(\%)

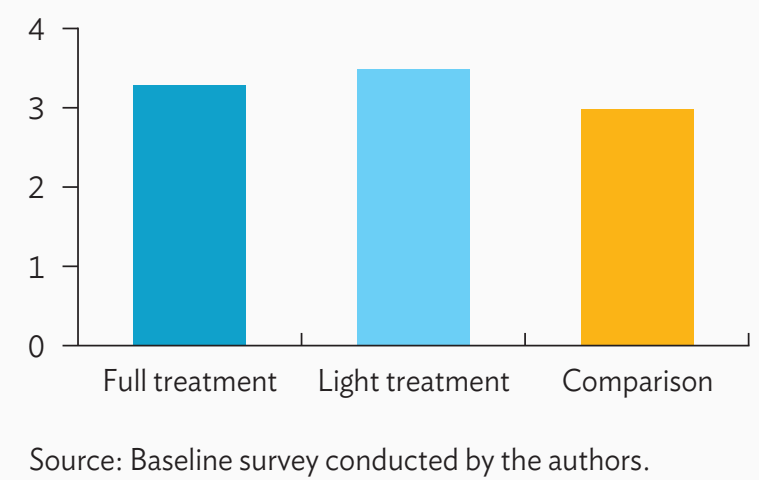


students responded negatively when asked about receiving support from their parents-with 15\%-17\% when asked about their parents' interest in school activities (Figure 39), and 11\%-13\% when asked about parental support at the time of physical or emotional difficulties (Figure 41).

\section{(vi) Feeling at Home}

Over 75\% of students responded liking their dormitory "very much" - 75\% from the full treatment group, 79\% from the light treatment group, and 77\% from the comparison group. Nearly $95 \%$ of the students liked their dormitories in general (Figure 43). Only $1 \%$ of the students answered that they "disliked" their dormitories. When asked to rank their general happiness using vignettes in the form of smiley faces, over $70 \%$ of students reported feeling "very happy." The proportion of students feeling "happy" accounted for $91 \%$ (Figure 44). ${ }^{14}$

\section{Figure 43: Students' Response to "How well do you like your school dormitory?"}

$(\%)$

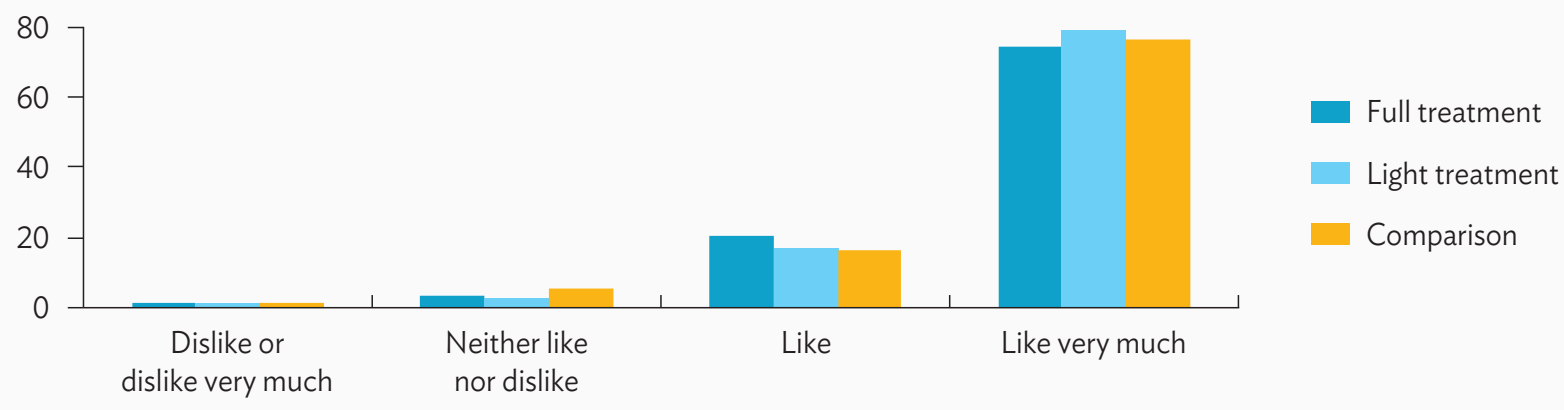

Source: Baseline survey conducted by the authors.

Figure 44: Students' Self-Rating on Their Happiness and Well-Being

$(\%)$

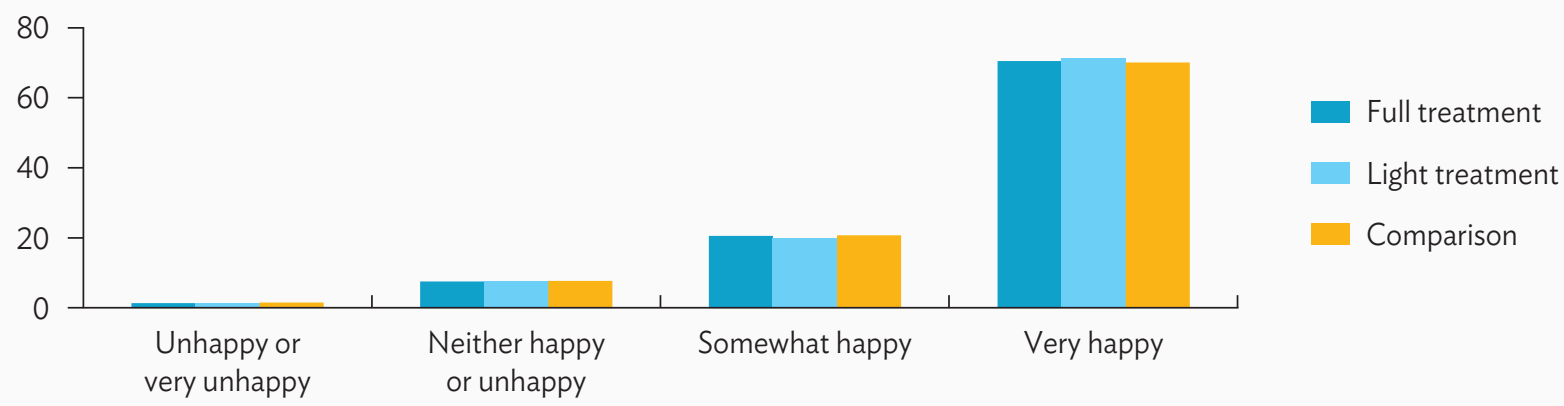

Source: Baseline survey conducted by the authors.

14 The vignettes are presented in Annex 6. 


\section{(vii) Students' Health}

Students were asked about their health condition 2 weeks prior to the survey. Twelve percent of the girls and $22 \%$ of the boys in the full treatment group reported being sick, $12 \%$ of the girls and $14 \%$ of the boys in the light treatment group also got sick, and 18\% of the girls and 17\% of the boys in the comparison group were also sick (Figure 45). The majority of those who got sick lasted for just 1 day or 2-3 days (Figure 46). Students who were sick for more than 3 days was $5 \%$ in the full treatment group, $16 \%$ in the light treatment group, and $17 \%$ in the comparison group.

\section{Figure 45: Dormitory Students Reporting Being Sick in the Last 2 Weeks}

(\%)

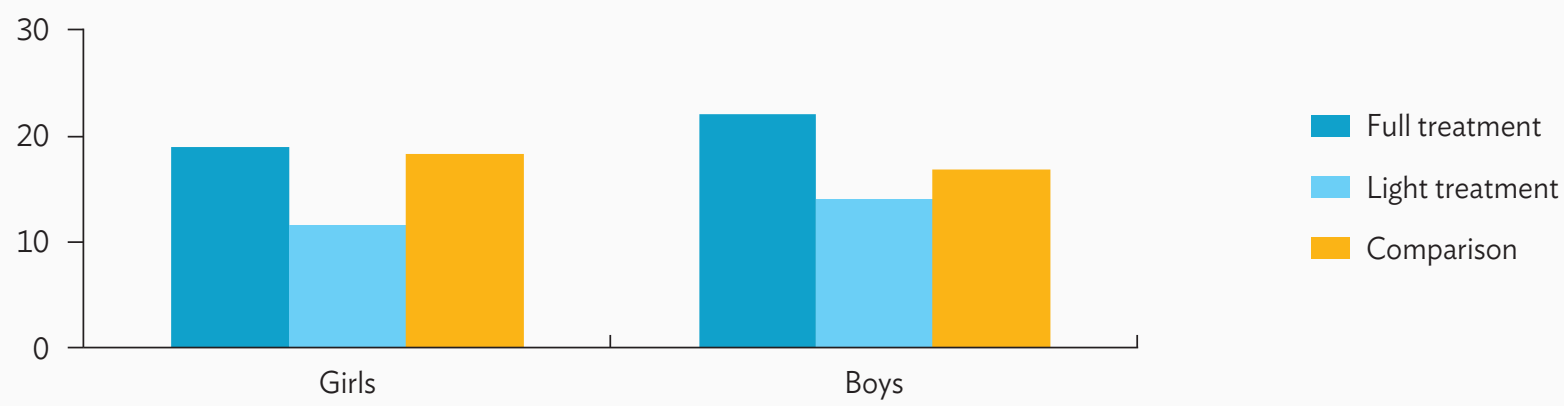

Source: Baseline survey conducted by the authors.

Figure 46: Number of Sick Days for Those Who Were Sick in the Last 2 Weeks

(\%)

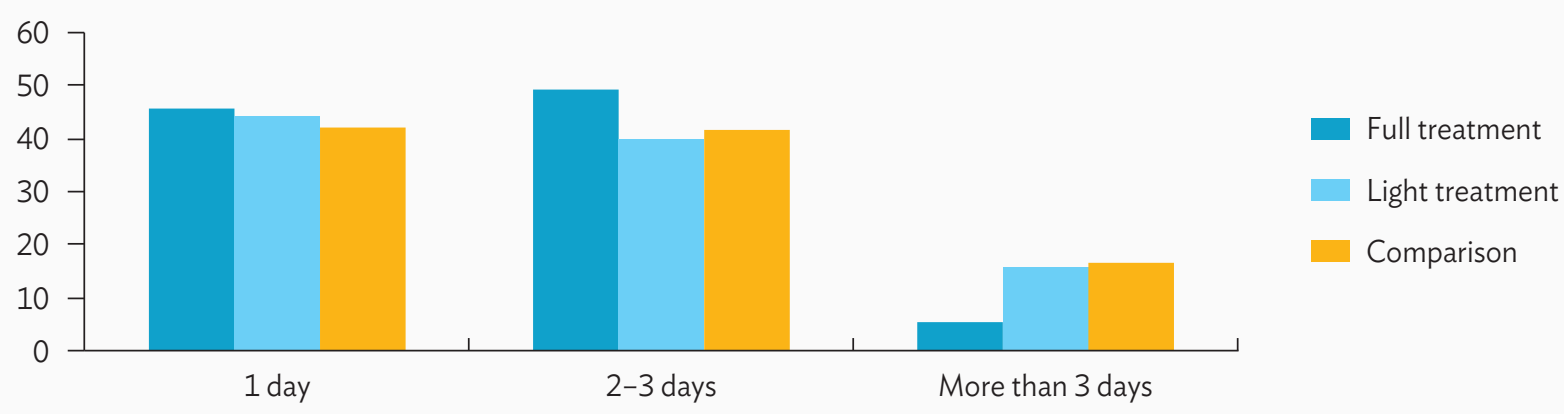

Source: Baseline survey conducted by the authors. 


\section{D | ADVANCED AND FINAL OUTCOMES}

Immediate and intermediate outcomes are expected to lead to a number of advanced and "final" outcomesthe latter referring to "hard" outcome measures, such as improved test scores, increased attendance, and reduced dropout rates (see the TOC in Annex 2). Except for test scores, robust calculations of the final outcomes at the level of treatment and comparison groups were not available at the time of baseline survey. This will need to be carried out in preparation for the endline survey. The findings below, therefore, present the advanced outcomes and student test scores only.

\section{(i) Parents' Attitude toward Placing Children in Dormitory}

The main reasons for placing their child in a dormitory were because parents could not possibly set up a ger or entrust their children to relatives living near the school. ${ }^{15}$ Only $11 \%$ of parents in the full treatment and light treatment groups reported that their children would be comfortable and safe at dormitories, compared to $21 \%$ in the comparison group (Figure 47 ). Also, only $10 \%$ of parents in the full treatment and light treatment groups responded "good physical environment" as their reason for placing their child in a dormitory, compared to $7 \%$ in the comparison group.

\section{Figure 47: Reasons for Placing Child in a Dormitory}

$(\%)$

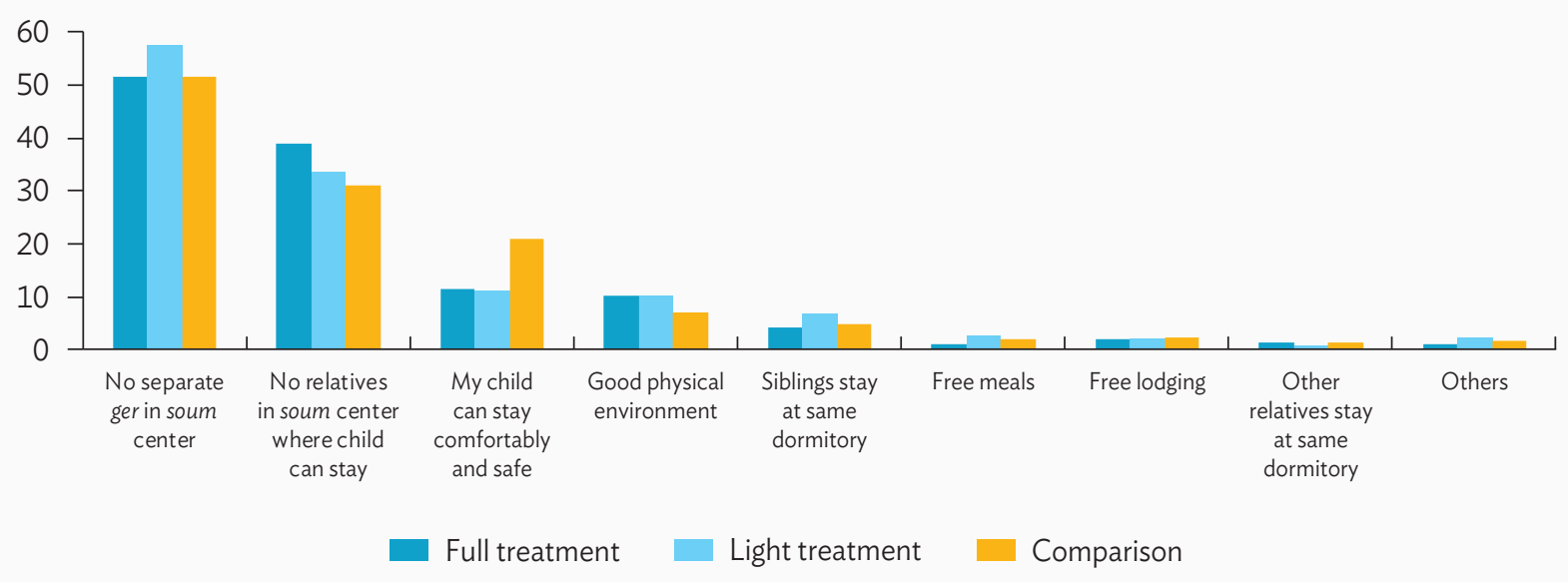

Source: Baseline survey conducted by the authors.

15 A ger is a traditional tent where seminomadic herders live. 


\section{(ii) School Attendance}

Among the students, $17 \%$ in full treatment and comparison groups, and 13\% in the light treatment group reported having missed at least 1 school day during the 2 weeks prior to the survey (Figure 48). Over half of these students who missed school did so once or $2-3$ times, with $2 \%-3 \%$ missing more than three times (Figure 49). These numbers corresponded well to the proportion of students reporting being sick in the last 2 weeks and their number of sick days. This also confirms that the main reason for missing school was sickness.

\section{Figure 48: Students Missing at Least 1 School Day in the Last 2 Weeks}

$(\%)$

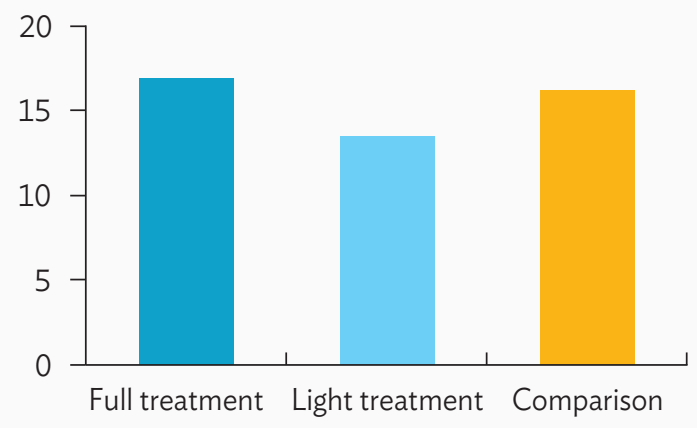

Source: Baseline survey conducted by the authors.
Figure 49: Frequency of Missing School in the Last 2 Weeks

(\%)

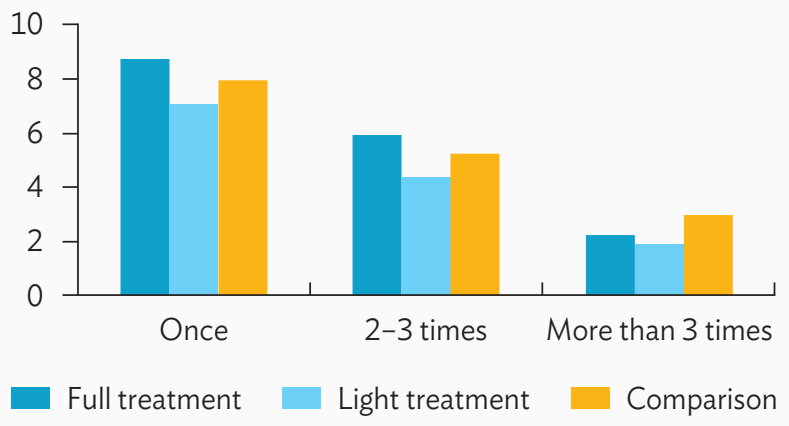

Source: Baseline survey conducted by the authors.

The proportion of students who were late for school at least once during the 2 weeks prior to the survey was similar across the three groups, at 20\%-23\% (Figure 50). Almost all students across the three groups reported doing their homework-97\% in the full treatment and light treatment groups, and $96 \%$ in the comparison group (Figure 51).

Figure 50: Children Who Were Late for School in the Last 2 Weeks

$(\%)$

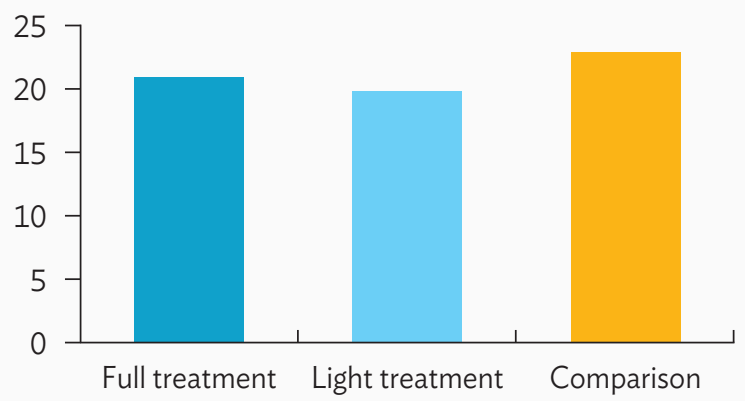

Source: Baseline survey conducted by the authors.
Figure 51: Children Who Did Their Homework Every Day

(\%)

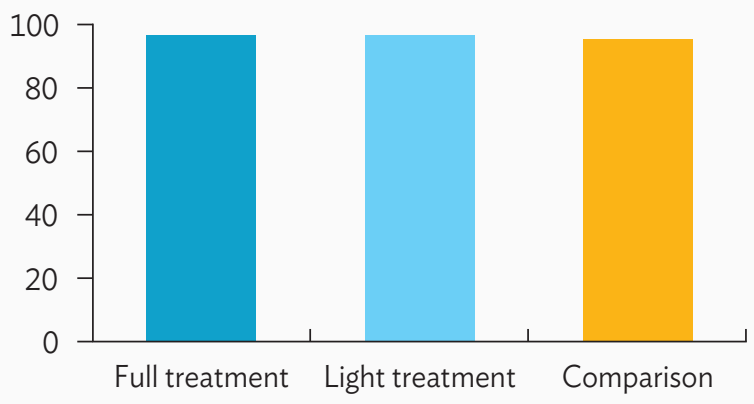

Source: Baseline survey conducted by the authors. 


\section{(iii) Test Scores}

In the treatment and comparison aimags, 5,209 5th grade students were staying in dormitories, of whom 674 were included in the survey. Of them, the registration numbers of 669 students were successfully matched with the scores of the national 5 th grade student assessment (i.e., math, Mongolian language, and science). The average scores in math, Mongolian language, and science of students staying in dormitories in the full treatment group were the highest, followed closely by those in the light treatment group (Figure 52). Students staying at comparison dormitories scored, on average, 8 percentage points lower than those in the full treatment group in math and science; and 6 percentage points lower in Mongolian language.

\section{Figure 52: Average 5th Grade Test Scores of Students Staying in Dormitories}

$$
(\mathrm{N}=669)
$$

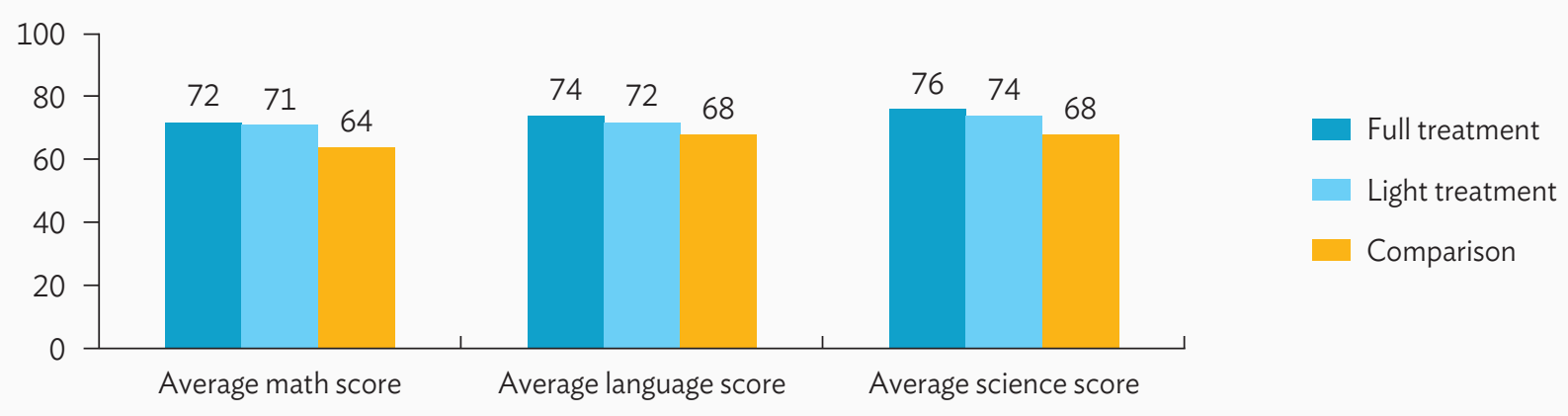

Source: Baseline survey conducted by the authors.

Figure 53 shows the proportion of 5 th grade students staying at dormitories in the treatment and comparison aimags who obtained "excellent" or "good" scores. Figure 54 presents all 5 th grade students (including students living in dormitories and living at home and elsewhere, with different socioeconomic backgrounds) in the treatment and comparison aimags who obtained "excellent" or "good" scores.

Figure 53: Students Obtaining “Excellent" or "Good" Scores: Students Staying in Dormitories, Sample Only

$(\%)$

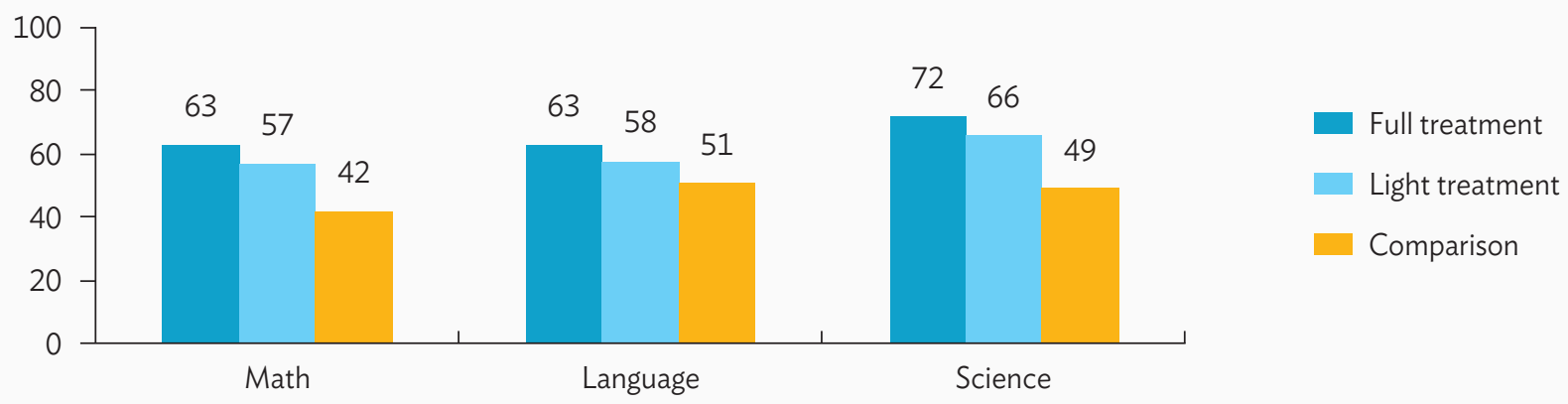

Source: Baseline survey conducted by the authors. 
Figure 54: Students Obtaining "Excellent” or “Good” Scores:

All 5th Grade Students in Survey Aimags (\%)

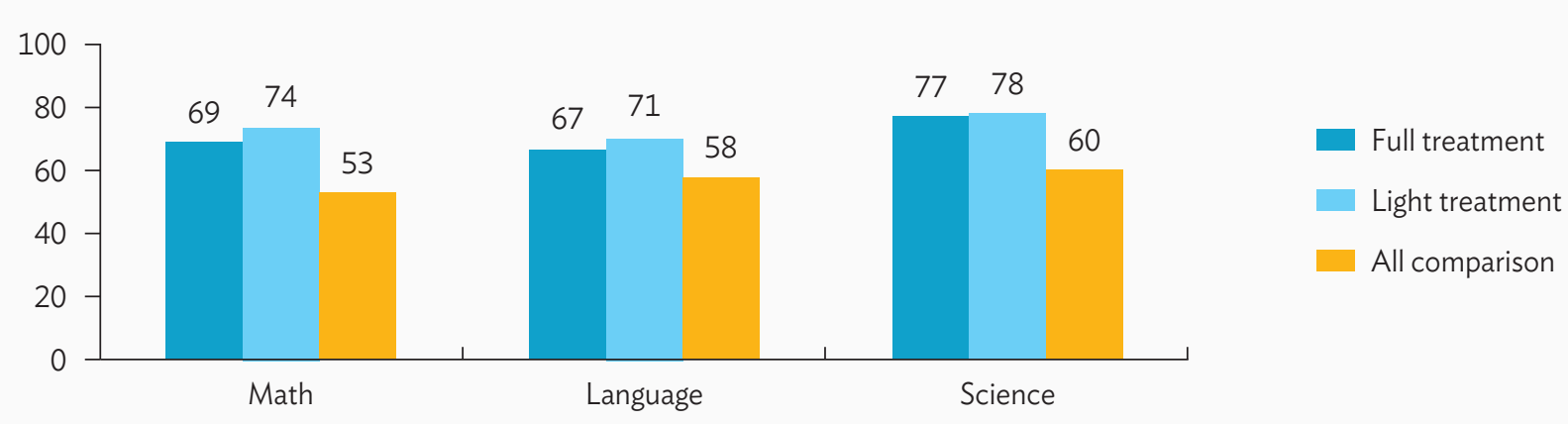

Source: Baseline survey conducted by the authors.

Students staying at full treatment dormitories recorded the best scores (Figure 53), although when all 5th grade students were included, students in light treatment schools appeared to do better (Figure 54). Overall, the average scores in the treatment and comparison aimags increased when students living at home and elsewhere were included. Students at the full treatment schools who obtained "excellent" or "good" scores increased by 6 percentage points in math, 4 percentage points in Mongolian language, and 5 percentage points in science when all 5 th grade students were included. For those at the light treatment schools, the difference was even larger-increasing by 17 percentage points in math, 13 percentage points in Mongolian language, and 12 percentage points in science. 
T he baseline survey examined the situation in dormitories, schools, and the circumstances surrounding the respondents in the full and light treatment, and comparison groups prior to the start of the project interventions. The survey focused on the following: (i) physical school dormitory environment, (ii) capacity to deliver dormitory services, and (iii) some of the advanced and final outcomes. In brief, a selection bias is present and can be observed as differences in student characteristics and in the physical dormitory environment. These are expected to be mitigated through econometric techniques before and during the endline survey.

This section summarizes the key findings of the baseline survey and provides recommendations for the endline survey to be conducted by the baseline survey team, in collaboration with MECSS, in SY2020/21. ${ }^{16}$

\section{A I BACKGROUNDS OF THE RESPONDENTS}

The parents' backgrounds differed among the treatment and comparison groups on several fronts, as discussed below:

Almost all parents surveyed were herders, with a slightly lower proportion in the comparison group. The most common level of educational attainment across parents in all the three groups was lower secondary education, with parents in the full treatment group having the lowest level of educational attainment on average, followed by parents in the light treatment group. Parents without education was noticeably fewer among those in the comparison group-they were more likely to have completed upper secondary education. Mothers, on average, were better educated than fathers across all three groups.

- Income levels of parents in the full treatment group were the lowest, with an average annual income of MNT6,875,000 (\$2,862) - which was $\$ 280$ less than those in the light treatment group, and $\$ 366$ less than those in the comparison group. ${ }^{17}$

- On average, parents of students in the full treatment group were poorer and attained lower levels of education than parents of students in the light treatment or comparison group. They were also more likely to be herders and dependent on childcare subsidies and government allowances. As the socioeconomic backgrounds of parents are well-established predictor of student learning outcomes, these baseline differences should be controlled and, to the extent possible, minimized at endline through propensity score matching.

16 The authors of this report mainly constituted the baseline survey team.

17 The official exchange rate of the Central Bank of Mongolia on 29 April 2018 was $\$ 1=$ MNT2,401.65. 


\section{B | PHYSICAL SCHOOL DORMITORY ENVIRONMENT}

Physical school dormitory environment also differed among the full treatment, light treatment, and comparison groups.

Dormitories in the full treatment group were, on average, constructed earlier than the other dormitories. They were also less likely to have undergone previous renovation, although $72 \%$ of these had at least undergone one major renovation.

Survey findings clearly illustrate the relevance of the planned project activities. Dormitories often lacked adequate WASH facilities, heating systems, and protection from precipitation and wind. Students staying at dormitories in the full treatment group were more likely to report feeling cold in winter (20\%), experienced leaking dormitory roofs (16\%), or felt wind blowing through dormitory windows (33\%). A smaller proportion of students in the light treatment and comparison groups reported experiencing these problems.

Many WASH facilities needed improvement, especially for early-grade students, girls, and students with disabilities. Most dormitory latrines were located outside, with less than $40 \%$ of dormitories across all three groups having indoor latrines. More than half of outdoor latrines were rated by enumerators as unsuitable for early-grade students, and over $80 \%$ of these were not suited for students with disabilities. Disability access was particularly poor in the full treatment group, which also had the poorest condition of toilets for girls.

Hygiene practices among dormitory students were similar across all three groups and needed ample scope for improvement to attain potential health benefits. About $40 \%$ of students in all three groups never or hardly ever washed hands after going to the toilet, and never or hardly ever showered once a week.

Libraries and a quiet place to study were less common in the full treatment group dormitories than in other dormitories. In the full treatment group, $43 \%$ of students had a quiet place to study, as compared to $56 \%$ of students in light treatment and comparison groups. Nearly half of students responded having "other students" bothering them when they wanted to study, indicating the need for quiet study spots with potential gains in learning outcomes.

\section{C | CAPACITY TO DELIVERY DORMITORY SERVICES}

\section{(i) Training of Dormitory Staff}

Many dormitory teachers and cooks had undergone training at the time of the baseline survey. Those in the treatment groups were more likely to have undergone training than those in the comparison group. A smaller proportion of dormitory guards had received training, with minor variations in number between the groups.

One-third of dormitory teachers in the treatment groups reported having undergone training on child-centered methodology. More than half had already received training on child violence and conflict resolution prior to the baseline survey. Dormitory teachers in the comparison group were less likely to have undergone these two types of training. 
Over one-third of cooks reported having received training on meal preparation, food nutritional value, or food safety. The highest proportion (about 50\%) of cooks in the light treatment group have undergone training.

(ii) Outreach and Communication Activities for Parents

The number of teachers with a plan to work with parents differed substantially among the groups. Less than half of teachers in the full treatment group planned to work with parents while more than two-thirds of teachers signified such intention in the light treatment group.

- Over $90 \%$ of parents across all three groups reported being aware of parent-teacher meetings that took place during SY2016/17, although only about half attended at least once. Around $40 \%$ of parents did not participate in any school-organized activities for parents, while the full treatment group reported a higher proportion of nonparticipants.

- Nearly $80 \%$ of students in all three groups reported parents' support for both educational and noneducational matters. A smaller proportion of students strongly disagreed, disagreed, or neither agreed nor disagreed when they were asked whether their parents support them (17\% in the full treatment group on parents' interest in school activities, and $12 \%$ on parents' support at the time of physical or emotional difficulties). The link between parents' support and final outcomes could be explored at endline by looking specifically at the subgroup of students who responded negatively on parents' support.

(iii) Student Committee and After-School Activities

Almost all dormitories had student committees, and about $40 \%$ of dormitory teachers in all three groups reported organizing learning activities jointly with student committees. Nearly $40 \%$ of the students (girls and boys) across all three groups engaged in after-school activities. The main reasons for not participating were being "not interested" (40\%), followed by "no after-school activities available" (20\%).

\section{D | ADVANCED AND FINAL OUTCOMES}

Most parents placed their children in dormitories by necessity. Some $11 \%$ of parents in the treatment groups thought that their children would be comfortable and safe in a dormitory. Parents in the comparison group were twice as likely to cite child comfort and safety as the reasons for placing their children in a dormitory compared to those in the treatment groups.

Most students living in dormitories missed school largely because of sickness. The proportion of students reporting having missed school corresponded well with those who reported being sick during the 2 weeks prior to the survey.

One in five students across all three groups answered having arrived late for school during the 2 weeks prior to the survey. Almost all students responded doing their homework every day. 
Students staying at dormitories in the treatment groups did better in the national 5 th grade student assessment in the areas of math, Mongolian language, and science than those in the comparison group. Students staying at dormitories in the full treatment group did better than those in the light treatment group.

- The average scores in the national 5th grade student assessment increased among the treatment and comparison aimags when students living at home and elsewhere were included. This indicates the scope of possibly improving the performance of students living in dormitories. When students living at home and elsewhere were included, the average scores at the light treatment schools were higher than those at the full treatment schools.

\section{E | RECOMMENDATIONS}

A number of steps and considerations are recommended to prepare for the endline survey that will be conducted by the baseline survey team in collaboration with MECSS in SY2020/21. These include the following areas:

\section{(i) Data Analysis}

- Some final outcome values at baseline (i.e., net intake rate in 1st grade, dropout rates, primary completion rate, gross intake rate in final grade of primary education, and junior secondary transition rate) should be calculated for each treatment and comparison group, using administrative data.

- Propensity score matching may be conducted on the baseline data to determine the size of "common support" and the extent to which such matching can eliminate part of the selection bias observed at baseline.

\section{(ii) Survey Design}

The survey questionnaires should be amended to (i) include specific questions about the knowledge and practices expected to be obtained through different types of training for dormitory teachers, guards, and cooks based on the final training materials; and (ii) capture a more nuanced understanding of the nature and scope of after-school "extracurricular activities." The survey should also reflect other activities in the treatment and comparison aimags, which are likely to affect the outcomes measured in the impact evaluation, including students' well-being and learning outcomes.

- Qualitative data collection methods that use participatory approaches and in-depth conversations with students and staff may be conducted to explore hard-to-measure outcomes, such as a child's well-being, social adjustment, and the nature and importance of staff-student relationship.

\section{(iii) Field Survey}

Sufficient resources, in terms of funds and time, should be allocated for the endline survey to ensure comprehensive enumerator training, piloting, and improved ability to interview parents or guardians, and in obtaining parental consent for child interviews. 


\section{REFERENCES}

Adukia, A. 2014. Sanitation and Education. Unpublished working paper. (The 2016 version was retrieved from http://home.uchicago.edu/adukia).

Angelini, V., D. Cavapozzi, L. Corazzini, and O. Paccagnella. 2014. Do Danes and Italians Rate Life Satisfaction in the Same Way? Using Vignettes to Correct for Individual-Specific Scale Biases. Oxford Bulletin of Economics and Statistics. 76(5). 0305-9049.

Asian Development Bank (ADB). 2015. Report and Recommendation of the President to the Board of Directors: Proposed Administration of Grant to Mongolia for Improving School Dormitory Environment for Primary Students in Western Region Project. Manila.

Borkum, E., F. He, and L. L. Linden. 2012. The Effects of School Libraries on Language Skills: Evidence from a Randomized Controlled Trial in India. NBER Working Paper No. w18183. https://www.nber.org/ papers/w18183.pdf.

Burde, D. and L. L. Linden. 2013. Bringing Education to Afghan Girls: A Randomized Controlled Trial of Village-Based Schools. American Economic Journal-Applied Economics. 5(3). pp. 27-40.

Cash, C. 1993. Building Condition and Student Achievement and Behavior. Unpublished doctoral dissertation. Virginia Polytechnic Institute and State University.

Dumitrescu, A., D. Levy, C. Orfield, and M. Sloan. 2011. Impact Evaluation of Niger's IMAGINE Program. Washington, DC: Mathematica Policy Research.

Education Evaluation Center of Mongolia. 2008. Mongolian National Assessment of Primary Education Mathematics and Reading. Ulaanbaatar.

Edwards, M. 1992. Building Conditions, Parental Involvement, and Student Achievement in the D.C. Public School System. Unpublished master's thesis. Georgetown University.

Freeman, M. C., L. E. Greene, R. Dreibelbis, S. Saboori, R. Muga, B. Brumback, and R. Rheingans. 2012. Assessing the Impact of a School-Based Water Treatment, Hygiene and Sanitation Programme on Pupil Absence in Nyanza Province, Kenya: A Cluster-Randomized Trial. Tropical Medicine \& International Health. 17(3). pp. 380-391.

Jeynes, W. 2007. The Relationship between Parental Involvement and Urban Secondary School Student Academic Achievement: A Meta-Analysis. Urban Education. 42(1). pp. 82-110.

Kapteyn, A., J. P. Smith, and A. Soest. 2007. Vignettes and Self-Reports of Work Disability in the United States and the Netherlands. American Economic Review. 97(1). pp. 461-473.

King, G., C. J. L. Murray, J. A. Salomon, and A. Tandon. 2004. Enhancing the Validity and Cross-Cultural Comparability of Measurement in Survey Research. American Political Science Review. 98(1).

pp. 191-207. 
Kristensen, N. and E. Johansson. 2008. New Evidence on Cross-Country Differences in Job Satisfaction Using Anchoring Vignettes. Labour Economics. 15: 96-117.

Lokshin, M. and M. Yemtsov. 2004. Combining Longitudinal Household and Community Surveys for Evaluation of Social Transfers: Infrastructure Rehabilitation Projects in Rural Georgia. Journal of Human Development. 5(2). pp. 265-277.

Masset, E. 2015. Measuring Empowerment in Rural India Using Vignettes. Journal of Development Effectiveness. 7(3). 346-356.

Ministry of Education, Culture, and Science of Mongolia. 2012. Pre-School, Primary and Secondary Education Reform Policy Framework, 2012-2016. Ulaanbaatar.

Newman, J., M. Pradhan, L. Rawlings, G. Ridder, R. Coa, and J. L. Evia. 2002. An Impact Evaluation of Education, Health, and Water Supply Investments by the Bolivian Social Investment Fund. World Bank Economic Review. 16(2). pp. 241-274.

Rice, N., S. Robone, and P. Smith. 2011. Analysis of the Validity of the Vignette Approach to Correct for Heterogeneity in Reporting Health System Responsiveness. European Journal of Health Economics. 12. pp. 141-162.

Snilstveit, B., J. Stevenson, D. Phillips, M. Vojtkova, E. Gallagher, T. Schmidt, H. Jobse, M. Geelen, M. Pastorello, and J. Eyers. 2015. Interventions for Improving Learning Outcomes and Access to Education in Low- and Middle-Income Countries: A Systematic Review, 3ie Final Review. London: International Initiative for Impact Evaluation (3ie).

Stewart, E. 2008. School Structural Characteristics, Student Effort, Peer Associations, and Parental Involvement: The Influence of School- and Individual-Level Factors on Academic Achievement. Education and Urban Society. 40(2). pp. 179-204.

White, H. and D. Raitzer. 2017. Impact Evaluation of Development Interventions: A Practical Guide. Manila: Asian Development Bank.

Yue, A., Y. Shi, F. Chang, C. Yang, H. Wang, H. Yi, R. Luo, C. Liu, L. Zhang, J. Chu, and S. Rozelle. 2012. Dormitory Management and Boarding Students in China's Rural Elementary Schools. REAP Working Paper 242. https://papers.ssrn.com/sol3/papers.cfm?abstract_id=2937676. 


\section{ANNEX 1 PROJECT OVERVIEW}

T he project-Improving School Dormitory Environment for Primary Students in Western Region Projectwill support the reform of the school dormitory system in Mongolia to ensure equal access to quality education for all students, particularly those from herder families who live a nomadic life, by establishing models to improve physical dormitory environment and services. ${ }^{1}$

The expected impacts of the project are (i) a reformed school dormitory system, (ii) a more equitable access to quality education, and (iii) the achievement of a universal primary education. This will be done by establishing and implementing models that can improve the physical environment and services of school dormitories in three aimags of the western region-Govi-Altai, Uvs, and Zavkhan. The project's overall outcome will be the implementation of these models, to be achieved through three primary outputs, as follows:

Output 1: Physical school dormitory environment improved. The project will improve the dormitory environment by supporting minor civil works for soum school dormitories in selected aimags. Civil works will include the (i) rehabilitation of buildings to adequately cope with winter temperatures, wind, and precipitation; (ii) repair and installation of universal design WASH facilities; (iii) provision of beds and other furniture to accommodate more students; and (iv) renovation of common rooms that will be properly furnished for study, reading, and extracurricular activities.

Output 2: Capacity to deliver school dormitory services improved. The project will support the capacity development of school dormitory staff and primary class teachers, school management and accountants, and aimag education department staff. The aim is to improve school dormitory services for primary students who are staying in dormitories, and their parents in the three project aimags. Dormitory teachers will be trained in child abuse issues and child-centered methods to facilitate study, reading, and extracurricular activities; and improve school preparedness of students, especially those in the early grades. Dormitory guards will also undergo trainings on child abuse and child-centered communication to support the students' study, reading, and extracurricular activities. Such trainings will enable dormitory guards to care for dormitory students at nighttime, especially early-grade students. The staff involved in preparing dormitory meals will similarly undergo training on the nutritional requirements of students. School management, accountants, and aimag education department staff will be trained to use tools and reference materials to enhance their management of financial, human, and physical resources of school dormitories.

ADB. 2015. Report and Recommendation of the President to the Board of Directors: Proposed Administration of Grant to Mongolia for Improving School Dormitory Environment for Primary Students in Western Region Project. Manila. https://www.adb.org/ projects/49168-001/main\#project-overview. 
Workshops will be organized for dormitory teachers, primary class teachers, and social workers so they could exchange experiences regarding student- and teacher-organized study, reading, and extracurricular activities in the dormitories. Communication and outreach activities for parents of primary students staying in dormitories will also be organized.

\section{Output 3: Policy and regulatory frameworks to improve the school dormitory environment developed.} The project will support the development of policy and regulation frameworks to improve school dormitory environment. Comprehensive standards for dormitories will be developed based on models to improve the physical environment and services, and national and international good practices in boarding school management. Funding formulas for school dormitory meals will be revised, taking into account the age and nutritional requirements of students. To replicate the models nationwide, a national strategy and financing policy will be developed to improve the school dormitory environment, particularly at soum schools with greater involvement by the local government. The hiring and recruitment of qualified dormitory teachers will also be considered, along with the operation and maintenance of the physical dormitory environment.

\section{BASIC PROJECT DATA}

\begin{tabular}{ll} 
Approval & 26 November 2015 \\
\hline Effectivity Date & 16 March 2016 \\
\hline Closing & 30 September 2019 \\
\hline Grant Amount Total & $\$ 3$ million (Japan Fund for Poverty Reduction administered by the Asian Development Bank) \\
\hline Executing Agency & Ministry of Education, Culture, Science and Sports (MECSS) \\
\hline Implementing Agencies & MECSS; education departments of Govi-Altai, Uvs, and Zavkhan Aimags \\
\hline
\end{tabular}




\section{ANNEX 2}

\section{THEORY OF CHANGE}

Improvement
of capacity to
deliver school
dormitory
services

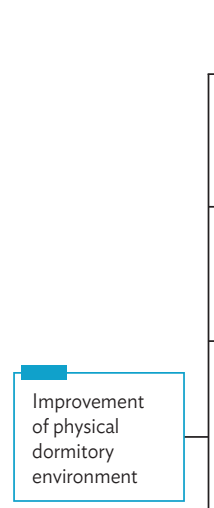

Interventions

Dormitories furnished with more beds, desks, chairs, and others.

\section{More dormitory rooms become usable. \\ Dormitories have common rooms (with computers and internet connection, books, textbooks, etc.) for study, reading, and extracurricular activities.}

Dormitory teachers trained in child abus issues and child-centered methods to

facilitate study, reading, and extracurricular activities, and improve school preparednes of students, especially in early grades.

Dormitory guards trained in child abuse issues and child-centered communication skills to encourage study, reading, and extracurricular activities, and care for dormitory students, especially in early grades, at night.

Student committee activities and student-organized study, reading, and extracurricular activities at dormitories promoted by dormitory and class teachers and social workers (school management).

Staff involved in the preparation of dormitory meals trained in nutritional requirements of students at different ages.

School management, accountants and management of financial, human, and physical resources for school dormitories.

Outreach and communication activities for parents of dormitory students at primary level more regularly conducted by school management and teachers. aimag education department staff trained on tools and reference materials to enhance
Children feel warmer, safer, and less disturbed with noise, leaks, and other problems that affect their daily lives.

Children can go to toilet more safely and comfortably whenever they want. Girls feel more comfortable managing their menstruation and attend classes. Children can acquire better hygiene practice. Children are cleaner and healthier.

\section{C}

Children can sleep comfortably and soundly. Children can do their homework and study when they want. Children can organize their personal items neatly and keep dormitory rooms organized and clean.

\section{Children can stay overnight at the dormitory} with their parents. and reading quietly. Children have place to engage and reading quietly. Children have place to enga
more with organized extracurriular activities.

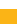

Dormitory teachers have better knowledge and skills to look after children's homework and study after school, organize activities to engage child
in study, reading and extracurricular activities, deal with fights, bullying, and problems among children at dormitories, and care about needs and well-being of each child. Dormitory guards have better knowledge and skills to encourage study, reading, and extracurricular activities, take care of small children, communicate with boys and girls from children's perspective, and handle emergency situations.

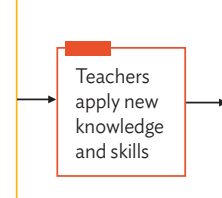

Children can safely and comfortably meet basic physical needs, feel more at home, and have better environment to engage with study, reading, and extracurricular activities. $\uparrow$

Student committee activities become more active in organizing study, reading, and extracurricular activities, and managing dormitory affairs.

Staffingo

Staff involved in the preparation of dormitory meals have better knowledge and skills to improve nutritional values of dormitory meals (buy and use different kinds of ingredients, use healthier cooking methods, etc.).

School management, accountants, and aimag education department staff have better

knowledge, skills, and tools to organize financial, human and physical resources for maintaining and improving physical dormitory environment and services. Parents of primary students staying in dormitories
have more information on their children's learning and school life and get interested in them.
Children have place to do their homework, study, after school, organize activities to engage children
Advanced

outcomes

Final/welfare

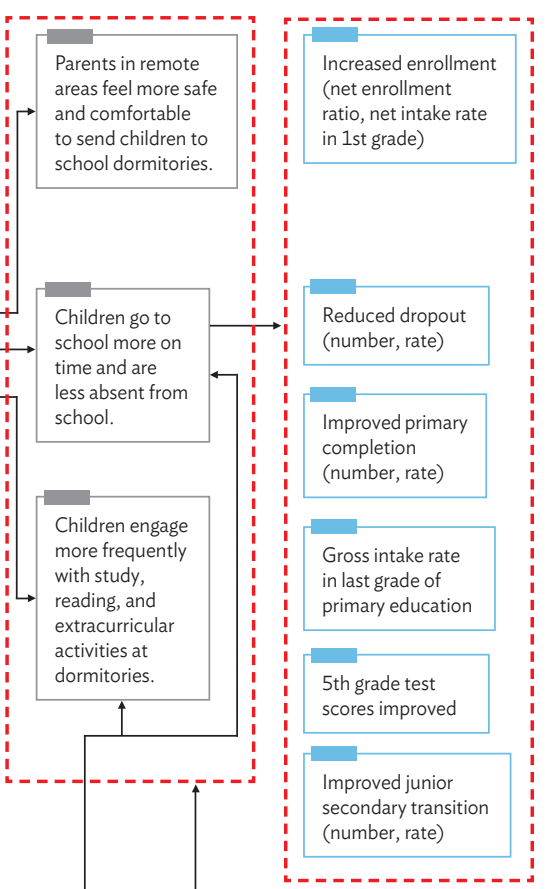

Source: Authors.
Children engage more frequently with study, with study,
reading, and extracurricular activities at dormitories. outcomes

outcomes

atio, net intake rate

1st grade)

Reduced dropout

number, rate)

mproved primary completion

Gross intake rate last grade of

th grade test scores improved

Improved junior (number, rate) 


\section{ANNEX 3}

\section{SUMMARY OF RELATED LITERATURE}

$T^{\text {hed }}$

he summary below draws on several sources. The description, outcome, length of follow up, study design, and sample size were reproduced from Table 6.6a in Snilstveit et al. (2015) with permission from the author (B. Snilstveit et al. 2015. Interventions for Improving Learning Outcomes and Access to Education in Lowand Middle-Income Countries: A Systematic Review. 3ie Systematic Review 24. London: International Initiative for Impact Evaluation [3ie]). The summary of findings was reproduced from study abstracts and/or executive summaries or results.

\section{STUDY:}

Assessing the Impact of a School-Based Water Treatment, Hygiene and Sanitation Program on Pupil Absence in Nyanza Province, Kenya: A Cluster-Randomized Trial

\section{SETTING:}

Hygiene infrastructure

Primary schools

Kenya

\section{DESCRIPTION:}

Two hygiene infrastructure interventions were tested:

(i) A hygiene promotion and water treatment arm that included a 3-day training of teachers on hygiene promotion, behavior change, and water treatment methods. Regular follow-up visits throughout the school year. The program provided handwashing and drinking water containers and a one-time, one-year supply of WaterGuard, a 1.2\% chlorine-based pointof-use water disinfectant.

(ii) Schools in the second intervention arm received components listed above, and latrines were provided. Students in both intervention and control schools received deworming treatment after the baseline, and another one subsequently.

\section{OUTCOMES:}

School attendance

\section{STUDY DESIGN:}

Cluster randomized controlled trial (RCT)

\section{LENGTH OF FOLLOW UP:}

17 months

\section{SAMPLE SIZE:}

6,036 students

\section{SUMMARY OF FINDINGS (FROM STUDY ABSTRACT OR RESULTS):}

No overall effect of the intervention on school absence was found. However, among schools in two geographical areas, those that received water treatment and hygiene promotion showed a 58\% reduction in absence for girls. In the same strata, sanitation improvement, in combination with water treatment and hygiene promotion, resulted in a comparable drop in school absence, although results were only marginally significant. Boys were not impacted by the intervention. In sum, school WASH improvements can improve school attendance for girls. Incomplete intervention compliance highlights the challenges of achieving consistent results across all settings.

\section{FULL REFERENCE:}

Freeman, M. C. et al. 2012. Assessing the Impact of a School-Based Water Treatment, Hygiene and Sanitation Programme on Pupil Absence in Nyanza Province, Kenya: A Cluster-Randomized Trial. Tropical Medicine \& International Health. 17(3). pp. 380-391. 


\section{STUDY:}

Sanitation and Education

\section{SETTING:}

- Hygiene infrastructure

Primary schools and upper primary schools

India

\section{DESCRIPTION:}

Using a national Indian school latrine construction initiative and administrative school-level data, the study explored whether the absence of school sanitation infrastructure impedes educational attainment, particularly among pubescent-age girls. The primary goal of the School Sanitation and Hygiene Education Programme was to increase school latrine coverage in rural areas to $100 \%$.

\section{OUTCOMES:}

Enrollment

Attendance (attended an exam) Dropout

\section{LENGTH OF FOLLOW UP:}

Up to 36 months (approx.) (depending on when latrines were built)

\section{STUDY DESIGN:}

Cost-benefit analysis (quasi-experiment with baseline and endline data collection)

\section{SAMPLE SIZE:}

121,206 primary schools

17,796 upper primary schools

\section{SUMMARY OF FINDINGS (FROM STUDY ABSTRACT OR RESULTS):}

School-latrine construction substantially increases the enrollment of pubescent-age girls, though mostly when providing sex-specific latrines. Any latrine substantially benefits younger girls and boys, who may be particularly vulnerable to sickness from uncontained waste. Academic test scores did not increase following latrine construction.

\section{FULL REFERENCE:}

Adukia, A. 2014. Sanitation and Education. Unpublished working paper. (The 2016 version was retrieved from http://home.uchicago.edu/adukia). 


\section{STUDY:}

Bringing Education to Afghan Girls: A Randomized Controlled Trial of Village-Based Schools

\section{SETTING:}

- Construction of new schools

Primary schools

Afghanistan

\section{DESCRIPTION:}

Partnership for Advancing Community-Based Education in Afghanistan (PACE-A): The program established community-based schools in rural villages to increase girls' primary participation in education by reducing the distance needed to go to school. Program partners provided teacher and community trainings, which was streamlined with the Ministry of Education's teacher training. Administrative support and materials were also provided. In some cases, supplies were provided to make the schools accessible and usable in winter.

\section{OUTCOMES:}

Attendance

Enrollment

Learning

\section{LENGTH OF FOLLOW UP:}

Two follow-ups: 4 months and 9 months (approx.)

\section{STUDY DESIGN:}

Cluster RCT

\section{SAMPLE SIZE:}

1,477 students (31 villages)

\section{SUMMARY OF FINDINGS (FROM STUDY ABSTRACT OR RESULTS):}

The program significantly increases enrollment and test scores among all children, but particularly for girls. Girls' enrollment increases by 52 percentage points and their average test scores increase by 0.65 standard deviations. The effect is large enough that it eliminates the gender gap in enrollment and dramatically reduces differences in test scores. Boys' enrollment increases by 35 percentage points, and average test scores increase by 0.40 standard deviations.

\section{FULL REFERENCE:}

Burde, D. and L. L. Linden. 2013. Bringing Education to Afghan Girls: A Randomized Controlled Trial of Village-Based Schools. American Economic Journal-Applied Economics. 5(3). pp. 27-40. 
STUDY:

The Effects of School Libraries on Language Skills: Evidence from a Randomized Controlled Trial in India

SETTING:

- Improvement and/or replacement of school infrastructure

Primary schools

India

\section{DESCRIPTION:}

The Akshara Library Program: This replaced existing libraries with ones that are well-equipped with books, designed to support the existing school curriculum, and staffed with a dedicated librarian. The librarian provided regular reading-focused educational activities to encourage use of the library and facilitate interaction using the available books. Akshara libraries were organized according to a hub and spoke system - with each hub school attached to several spoke schools in the same geographic area. Hub schools contain physical libraries while spoke schools do not have a physical library, instead, they are visited regularly by a mobile librarian.

\section{OUTCOMES:}

Attendance

Learning

LENGTH OF FOLLOW UP:

18 months (approx.)

STUDY DESIGN:

Cluster RCT

SAMPLE SIZE:

20,858 students

\section{SUMMARY OF FINDINGS (FROM STUDY ABSTRACT OR RESULTS):}

The program had no impact on students' scores on a language skills test administered after 16 months. The estimates are sufficiently precise to rule out effects larger than 0.053 and 0.037 standard deviations, based on the $95 \%$ and $90 \%$ confidence intervals. This finding is robust across individual competencies and subsets of the sample. The method of treatment, however, does seem to matter-physical libraries have no effect, while visiting librarians actually reduce test scores. The study finds no impact on test scores in other subjects or attendance rates.

\section{FULL REFERENCE:}

Borkum, E., F. He, and L. L. Linden. 2012. The Effects of School Libraries on Language Skills: Evidence from a Randomized Controlled Trial in India. NBER Working Paper No. w18183. 


\section{STUDY:}

An Impact Evaluation of Education, Health, and Water Supply Investments by the Bolivian Social Investment Fund

\section{SETTING:}

- Improvement and/or replacement of school infrastructure

- Primary and secondary schools

Bolivia

\section{DESCRIPTION:}

Social Investment Fund (SIF) Education investments: SIF-financed education projects either repaired existing schools or constructed new ones and provided new desks, blackboards, and playgrounds. New schools were often constructed in the same location as old schools, which were then used for storage or converted into housing for teachers. The wider SIF project consisted of a number of infrastructure and complementary measures in the areas of primary education, basic health care, and sanitation.

\section{OUTCOMES:}
Enrollment
Attendance
Completion
Dropout

\section{LENGTH OF FOLLOW UP:}

60 months (approx.)

\section{STUDY DESIGN:}

Two samples:

Chaco: Cluster RCT

Resto Rural: Cost-benefit analysis (quasi-experiment with baseline and endline data collection)

\section{SAMPLE SIZE:}

Chaco: 84 schools

Resto Rural: 64 schools

\section{SUMMARY OF FINDINGS (FROM STUDY ABSTRACT OR RESULTS):}

Results from education projects suggest that although they improved school infrastructure, they had little impact on education outcomes.

\section{FULL REFERENCE:}

Newman, J. et al. 2002. An Impact Evaluation of Education, Health, and Water Supply Investments by the Bolivian Social Investment Fund. World Bank Economic Review. 16(2). pp. 241-274. 


\section{STUDY:}

Combining Longitudinal Household and Community Surveys for Evaluation of Social Transfers:

Infrastructure Rehabilitation Projects in Rural Georgia

\section{SETTING:}

- Improvement and/or replacement of school infrastructure

- Georgia (rural) School level: not reported

Age: not reported

Grade: not reported

\section{DESCRIPTION:}

Investments typically involved the rehabilitation and repair of existing schools rather than construction of new ones.

These focused on improving school buildings-repairing roofs, windows, and floors, replacing inner pipes, installing sanitary and heating equipment, repainting walls, and the "weatherization" of infrastructure to make it usable during winter. These projects were part of a wider program on rural infrastructure rehabilitation projects in Georgia.

\section{OUTCOMES:}

Enrollment

Completion

\section{LENGTH OF FOLLOW UP:}

12 months (approx.) for enrollment outcome

48 months (approx.) for completion outcome

\section{STUDY DESIGN:}

Cost-benefit analysis (quasi-experiment with baseline and endline data collection)

\section{SAMPLE SIZE:}

249 villages

\section{SUMMARY OF FINDINGS (FROM STUDY ABSTRACT OR RESULTS):}

Changes in all outcome indicators unambiguously point to a positive long-term effect of the school project, particularly on pupils missing classes - which dropped in project villages by $6 \%$, while it increased by $2 \%$ in the matched control group. The health impact of the school rehabilitation is substantial. In the treatment group villages, the incidence of respiratory diseases among school-age children declined by $12 \%$, compared with only a $6 \%$ reduction in the villages without the project.

\section{FULL REFERENCE:}

Lokshin, M. and M. Yemtsov. 2004. Combining Longitudinal Household and Community Surveys for Evaluation of Social Transfers: Infrastructure Rehabilitation Projects in Rural Georgia. Journal of Human Development. 5(2). pp. 265-277. 
STUDY:

Impact Evaluation of Niger's Imagine Program

SETTING:

- Niger (rural) Primary schools

Age: 9 years

Grade: not reported

\section{DESCRIPTION:}

The program involved constructing 68 primary schools and implementing a set of complementary interventions. The schools were based on a prototype that included three classrooms, housing for three female teachers, a preschool, and separate latrines for boys and girls equipped with hand washing stations. The schools were located near a water source. The complementary interventions involved design and dissemination of training modules for teachers, supply of learning materials, promotion of extracurricular activities, teacher incentives, and a girls' education campaign. This latter component included advocacy days, local action plans, capacity building through school committees, adult literacy, and income-generating projects.

\section{OUTCOMES:}

- Learning

Enrollment

Attendance

\section{LENGTH OF FOLLOW UP:}

23 months

\section{STUDY DESIGN:}

Cluster RCT

\section{SAMPLE SIZE:}

Up to 13,969 students

\section{SUMMARY OF FINDINGS (FROM STUDY ABSTRACT OR RESULTS):}

IMAGINE had a 4.3 percentage point positive impact on primary school enrollment, no impact on attendance, and no impact on math and French test scores. The program impacts were generally larger for girls than for boys. For girls, the program had an 8 percentage point positive impact on enrollment, and a 5.4 percentage point impact on attendance. The program had no impact on girls' math scores, though evidence suggests it may have had a positive impact of 0.09 standard deviations on girls' French test scores. No significant impacts were detected for boys' enrollment, attendance, or test scores. Finally, impacts were larger for younger children (ages 7-10), than for those between the ages of 10 and 12.

\section{FULL REFERENCE:}

Dumitrescu, A., D. Levy, C. Orfield, and M. Sloan. 2011. Impact Evaluation of Niger's IMAGINE Program. Washington, DC: Mathematica Policy Research. 


\section{ANNEX 4}

\section{APPROACH AND METHODOLOGY}

$T^{T}$

his evaluation takes a theory-based approach. A theory-based impact evaluation lays out the project theory of change and measures not only impacts but explores how the project works at all stages-from inputs to outputs, to outcomes then to impact. This makes it possible to analyze which components or which phases of the project work well, and which ones do not. Weak links in the causal chain that prevent the achievement of impacts can be identified, while design features that need adjustment, for future phases of the project or for similar projects, can be pinpointed. Specifically, theory-based impact evaluation quantitatively measures outputs, outcomes, and impacts along the project causal chain.

\section{A. IMPACT EVALUATION OVERVIEW}

An impact evaluation examines the causal effects of a project or intervention. ${ }^{1}$ This is done by comparing project beneficiaries - the treatment group - to a similar, comparison group. A key challenge is to avoid or eliminate selection bias, which occurs when the treatment group is systematically and statistically different from a given control or comparison group. That will often be the case when the treatment group has not been randomly selected. For instance, projects frequently target either those groups that need it the most, or those with the highest potential for success. This is also the case in this project where (i) some of the country's poorest areas were identified, and (ii) the most dilapidated and needy dormitories within these aimags were selected to undergo physical upgrades.

To overcome selection bias, an impact evaluation can take an experimental or quasi-experimental approach. An experimental approach is the random assignment of treatment to a group of potential beneficiaries. If this can be done, it is generally the strongest methodological approach. Often however, randomization is not feasible, which is the case in this current project where the most dilapidated dormitories were specifically targeted for physical renovation, and where the participation of all dormitories in the three project aimags in capacity building components was predetermined. Therefore, a quasi-experimental approach is chosen, which seeks to mimic a randomized experiment to the best extent possible.

1 For a deeper understanding of impact evaluation concepts and the methodology described here, see White H. and D. Raitzer. 2017. Impact Evaluation of Development Interventions: A Practical Guide. Manila: ADB. 


\section{B. QUASI-EXPERIMENTAL APPROACH}

This impact evaluation applies a matched difference-in-differences approach. This is a quasi-experimental approach to impact evaluation - which estimates the average treatment effect on the treated by comparing treatment and comparison groups (first difference) over time (second difference). This is done by carrying out identical surveys just prior to project start (baseline) and after project end (endline).

Identical surveys will be completed at the same dormitories at baseline and endline and will cover the same number of children at each class level in the two survey rounds. ${ }^{2}$ All students who were covered in the 2017 baseline survey and remain in primary school at the time of the endline survey will be included in the second survey. This will enable the evaluator to examine average changes in key factors at the school level, and changes at the individual student level for those who were in 1st-3rd grades at baseline.

For the impact estimates to be valid, the so-called parallel trend assumption must generally hold true.

This assumes that the two groups would have developed at the same speed and direction in the absence of the project. This may not be the case if change over time is determined by unobserved characteristics of either group. A relevant example would be if external factors influence enrollment for the treatment schools so that these would change at a different rate than that of the comparison group even without the intervention. Such external factors could be greater population increase in the treatment area, closing of other schools in nearby areas, or others. One way to test the likelihood that there is a parallel trend is by examining past data on key outcome variables for the two groups.

In this present evaluation, no such data exist for the outcomes presented in this report. ${ }^{3}$ In cases where such data are not available, or where there is concern that the assumption may not be correct, the likelihood that the parallel trend assumption holds true can be strengthened by combining the difference-in-differences estimation with matching techniques. This evaluation uses propensity score matching to match treatment and comparison dormitories and students on baseline characteristics before estimating difference-indifferences results, applying what is referred to as a "matched difference-in-differences approach." Based on baseline characteristics, the matching will be carried out in preparation for the endline survey to guide the survey, and again, following endline data collection, to account for attrition of some participants.

\section{DEFINING TREATMENT GROUPS}

The unit of assignment (treatment) is the school dormitory. The unit of analysis is both dormitory and primary school students.

Exceptions may occur when the same number of students is no longer enrolled or attending each grade.

3 Time-series data for final outcomes on dropout rates and primary completion rates may be available from the MECSS to be used at endline survey. 
The project provides two intensities of treatment:

\section{a. Full treatment}

A total of 24 soum school dormitories receiving the full project support including (i) upgrading of physical school dormitory environment; (ii) training of dormitory staff; (iii) promotion of student committee activities and student-organized study, reading, and extracurricular activities in dormitories among school management and teachers; and (iv) promotion of outreach activities for parents with children living in dormitories, particularly those at the primary level, among school management and teachers, covering 6 dormitories in Govi-Altai, 13 in Uvs, and 5 in Zavkhan.

- Full treatment dormitories were identified by the project based on the following criteria:
number of primary students staying in school dormitories,
existence of junior and senior secondary grade classes,
year of dormitory construction and/or rehabilitation, and
unavailability of basic infrastructure (water, wastewater, and heating).

\section{B. Light treatment}

The remaining 54 dormitories in the three regions will receive partial treatment, with project support (ii)-(iv).

- This covers 16 dormitories in Govi-Altai, 18 in Uvs, and 20 in Zavkhan.

The evaluation will compare "full treatment" against "no treatment," and "light treatment" against "no treatment."

\section{IDENTIFICATION OF COMPARISON GROUP}

There were 517 dormitories in 21 aimags in the country in SY2016/17. Since all dormitories within the three project aimags were treated either "fully" or "lightly," comparison dormitories were identified from other aimags. To ensure that the comparison group was as identical as possible to the treatment group and, thus, optimizing the chances of a valid parallel trend assumption, a three-step process was carried out, as follows:

Step 1: Project aimags were matched with comparison aimags using both qualitative assessment and propensity score matching (PSM)-an econometric technique matching aimags based on the following key characteristics using data from the National Statistics Office of Mongolia:

rural population size,

proportion of students starting at 1st grade and who complete 5th grade,

labor force size, 
number of general education schools,

human development index score,

proportion of people living as herders, and

proportion of economically active people

Since the precision of PSM increases with sample size, and because there are only 21 aimags in Mongolia, the aimags with the best match were first identified based on PSM, and subsequently reviewed qualitatively by the baseline survey team.

Step 2: Comparison dormitories were sampled from a total of 116 dormitories in comparison aimags. This was done using a two-step approach:

(i) To ensure the inclusion of dormitories that were similar to the full treatment group, 24 dormitories from the comparison aimags were identified based on the project selection criteria used to identify the 24 full treatment project dormitories.

(ii) An additional group of 54 dormitories were randomly sampled from the remaining dormitories within the comparison aimags.

The optimal comparison groups for full treatment and light treatment, respectively, will be identified using PSM before and during the endline survey.

Step 3: Of the early-grade students, 18 were randomly sampled from within each treatment dormitory and 17 from within each comparison dormitory. Key staff were identified for interviews based on their role and position.

\section{E. SAMPLE OVERVIEW}

The sample consists of four groups: full treatment, light treatment, full comparison, and light comparison. For the purpose of the baseline study, however, results are presented by full treatment, light treatment, and comparison only. This is primarily because an initial review of baseline results did not overall show greater similarity between full treatment and full comparison than between full treatment and light comparison. Propensity score matching will be conducted at endline to construct an improved comparison group for the full treatment.

A total of 158 dormitories were covered by the survey. These consisted of all 78 dormitories in the three project aimags and 80 comparison dormitories. The treatment group was made up of 24 full treatment dormitories and 54 light treatment dormitories. The comparison group, although presented as one groupfor the purpose of presenting baseline results - consisted of 24 dormitories that matched the selection criteria for the 24 full treatment dormitories, and an additional 56 dormitories randomly sampled from the comparison aimags. Table A1 shows dormitories by aimag and by treatment group. 
Table A1: Dormitory Sample by Treatment Status and by Aimag

\begin{tabular}{|c|c|c|c|c|c|}
\hline Item & Full Treatment & Light Treatment & Full Comparison & Light Comparison & Total \\
\hline Govi-Altai & 6 & 15 & NA & NA & 21 \\
\hline Uvs & 13 & 19 & NA & NA & 32 \\
\hline Zavkhan & 5 & 20 & NA & NA & 25 \\
\hline Arkhangai & NA & NA & 9 & 17 & 26 \\
\hline Bayankhongor & NA & NA & 4 & 13 & 17 \\
\hline Bulgan & NA & NA & 3 & 15 & 18 \\
\hline Dundgovi & NA & NA & 5 & 4 & 9 \\
\hline Khentii & NA & NA & 3 & 7 & 10 \\
\hline TOTAL & 24 & 54 & 24 & 56 & 158 \\
\hline
\end{tabular}

NA = not applicable.

Source: Authors.

Within the 158 dormitories, 2,911 primary school students living in dormitories were interviewed, as well as 2,495 parents, 1,051 class teachers, 141 school principals, 157 dormitory teachers, 156 dormitory guards, and 144 cooks. Not all parents of student respondents were available for interview, which was partially addressed by interviewing a number of parents of students in the dormitories who were not surveyed. Table A2 shows the full sample of respondents and the number of parent-student matches.

Table A2: Overview of Sample by Survey Type and by Treatment Group

\begin{tabular}{l|c|c|c|c}
\hline Item & Full Treatment & Light Treatment & Comparison & Total \\
\hline Dormitories & 24 & 54 & 80 & 158 \\
\hline School principals & 20 & 51 & 70 & 141 \\
\hline Schoolteachers & 179 & 326 & 546 & 1,051 \\
\hline Dormitory teachers & 24 & 56 & 77 & 157 \\
\hline Dormitory guards & 26 & 53 & 77 & 156 \\
\hline Cooks & 21 & 52 & 71 & 144 \\
\hline Students & 538 & 893 & 1,480 & 2,911 \\
\hline Parents & 441 & 738 & 1,316 & 2,495 \\
\hline Student-parent match & 395 & 675 & 1,106 & 2,176 \\
\hline
\end{tabular}

Source: Authors. 
Figure A1: Distribution of Students Staying in Dormitories by Grade and \% of Students Interviewed

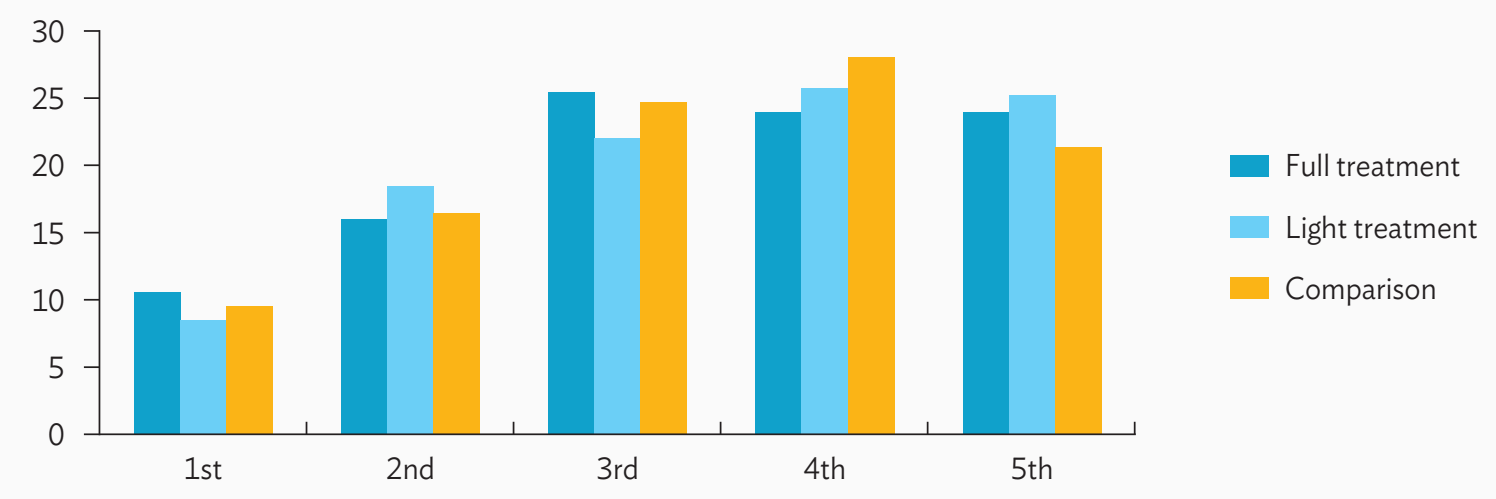

Source: Authors.

Figure A1 shows the distribution of the 2,911 students by grade. The intention was to draw a random sample of primary students across all five grades, but oftentimes, the number of primary students in the sampled dormitories was insufficient to draw a sample. Hence, all available students were selected for interview. Where more primary school students were found, such as in aimag center schools, students were sampled, prioritizing students for whom parental consent had already been obtained. The number of students attending 1 st-2nd grades was smaller than the number attending 3rd-5th grades, which was reflected in the sample size distribution.

\section{F. SURVEY TOOLS AND METHODS}

Survey instruments were developed based on the project design and the theory of change (Annex 2) to enable evaluators to answer the evaluation questions and hypotheses. Relevant literature and existing survey questionnaires used in Mongolia, such as the Household Socio-Economic Surveys, the Social Indicator Sample Surveys, the Population and Housing Census, and the Multiple Indicator Cluster Surveys were reviewed.

The following questionnaires were developed and will also be used for the endline survey to assess changes in the outcomes following the project completion. A detailed outline of each questionnaire and topics is presented in Table A3.

(i) Students' questionnaire

(ii) Dormitory staff's questionnaires (teachers, guards, and cooks)

(iii) School management's questionnaire

(iv) Parents' questionnaire

(v) Aimag education specialists' questionnaire 
Table A3: Survey Tools

\begin{tabular}{|c|c|}
\hline Questionnaire & Sections/Topics \\
\hline Students' Questionnaire & $\begin{array}{l}\text { A. Background Information } \\
\text { B. Dormitory Environment } \\
\text { C. Dormitory Learning Environment } \\
\text { D. School Well-Being } \\
\text { E. Dormitory Well-Being } \\
\text { F. Dormitory Food }\end{array}$ \\
\hline Parents' Questionnaire & $\begin{array}{l}\text { A. Background Information } \\
\text { B. Dormitory Well-Being } \\
\text { C. Parent and Child Relationship } \\
\text { D. Relationship between Parent and Dormitory Teacher, Guard } \\
\text { E. Information for Parents } \\
\text { F. Children's Disease and Sudden Illness }\end{array}$ \\
\hline Teachers' Questionnaire & $\begin{array}{l}\text { A. Background Information } \\
\text { Questions for Dormitory Teachers } \\
\text { B. Job Place Information } \\
\text { C. Working with Children and Student's Committee } \\
\text { D. Working with Parents } \\
\text { E. Professional Development Activities } \\
\text { Questions for Class Teachers } \\
\text { F. Job Place Information } \\
\text { G. Working with Parents } \\
\text { H. Professional Development Activities }\end{array}$ \\
\hline Dormitory Guards' Questionnaire & $\begin{array}{l}\text { A. Background Information } \\
\text { B. Job Place Information } \\
\text { C. Working with Students } \\
\text { D. Information and Relationship with Parents } \\
\text { E. Professional Development Activities }\end{array}$ \\
\hline Dormitory Cooks' Questionnaire & $\begin{array}{l}\text { A. Background Information } \\
\text { B. Information Related to Your Working Place } \\
\text { C. Meal Preparation } \\
\text { D. Professional Development Activities }\end{array}$ \\
\hline School Management's Questionnaire & $\begin{array}{l}\text { A. Background Information } \\
\text { B. School Information } \\
\text { C. Information about Dormitory Building and Equipment } \\
\text { D. Dormitory Human Resource Information } \\
\text { E. Dormitory Financial Resource Information } \\
\text { F. Feeling about Dormitory and Dormitory Services } \\
\text { G. Questions for the Primary Education Training Manager } \\
\text { H. Questions for the School Social Worker } \\
\text { I. Questions for the Accountant } \\
\text { J. Questions for the Soum Governor }\end{array}$ \\
\hline Aimag Education Specialists' Questionnaire & $\begin{array}{l}\text { A. Background Information } \\
\text { B. Support for Dormitories } \\
\text { C. Budget and Finance Dormitory } \\
\text { D. Professional Development Activities }\end{array}$ \\
\hline
\end{tabular}


The baseline survey was designed to capture not only project outputs and outcomes but also the final welfare outcomes-including the psychological well-being of students staying in dormitories. To measure "hard-tomeasure" outputs and outcomes, the survey tools included a number of innovative features, as follows:

The dormitory staff's questionnaire included enumerator assessments on dormitory facilities for which enumerators had been trained to apply specific criteria.

The student survey tool included vignettes in the forms of smiley faces to help children express their feelings or opinions on a number of questions. The vignettes are found in Annex 6.

To measure psychological child well-being, the survey employed anchoring vignettes in the form of small descriptions of primary school children with different levels of well-being, allowing student to compare and rank themselves using the vignettes. Anchoring vignettes is a tool that is most often used (i) to measure complicated concepts that do not have objective numerical measurements, and (ii) to ensure comparability of self-assessments responses across individuals and population groups. The approach is best known for its use in the World Health Organization's World Health Surveyon questions of perceived health, and in the Programme for International Student Assessment of the Organisation for Economic Co-operation and Development - to assess issues, such as level of teacher support and teacher management performance. It was similarly used to measure such diverse concepts as political efficacy, disability, job satisfaction, public sector performance, and life satisfaction, and tested to measure women's empowerment in India. ${ }^{4}$ Results from those "hard-to-measure" outputs and outcomes will be analyzed at endline.

\section{G. SAMPLING AND POWER CALCULATIONS}

A two-stage cluster sampling approach was applied, selecting first dormitories (clusters), then drawing a random sample of individual students within each dormitory.

When calculating sample size for a survey or evaluation with several outcomes of interest, priorities must be made in terms of what indicators are most important, to be able to measure them. Where data are available, the choice is often made to calculate the sample size needed for each outcome of interest, and subject to logistics and budget, settle on the largest sample size required. In that case, all other indicators will be measured with the same or greater precision. In the case of this evaluation, past data on outcomes

4 King, G., C. J. L. Murray, J. A. Salomon, and A. Tandon. 2004. Enhancing the Validity and Cross-Cultural Comparability of Measurement in Survey Research. American Political Science Review. 98(1). pp. 191-207; Kapteyn, A., J. P. Smith, and A. Soest. 2007. Vignettes and Self-Reports of Work Disability in the United States and the Netherlands. American Economic Review. 97(1). pp. 461-473; Kristensen, N. and E. Johansson. 2008. New Evidence on Cross-Country Differences in Job Satisfaction Using Anchoring Vignettes. Labour Economics. 15: 96-117; Rice, N., S. Robone, and P. Smith. 2011. Analysis of the Validity of the Vignette Approach to Correct for Heterogeneity in Reporting Health System Responsiveness. European Journal of Health Economics. 12. pp. 141-162; Angelini, V., D. Cavapozzi, L. Corazzini, and O. Paccagnella. 2014. Do Danes and Italians Rate Life Satisfaction in the Same Way? Using Vignettes to Correct for Individual-Specific Scale Biases. Oxford Bulletin of Economics and Statistics. 76(5). 0305-9049; Masset, E. 2015. Measuring Empowerment in Rural India Using Vignettes. Journal of Development Effectiveness. 7(3). 346-356. 
of interest were not available at the time of sample size calculations. The choice, therefore, was to focus on student test scores, which is a final outcome measured at the student level and an outcome frequently used in education programs aimed at improving learning outcomes. Although previous data on test scores were not available, sample size calculations were completed based on data from related studies elsewhere.

The following parameters are needed to estimate sample size:

Minimal effect size, also called minimal detectable effect. This reflects the size of the effect expected to be able to measure (the difference in means between project and comparison group).

- The desired significance level $(\alpha)$. This is the level of certainty with which the results become reliable. The standard is generally 0.05 .

- The power of the test. This is the probability at which a statistically significant result will be detected and, hence, that the test will correctly lead to the rejection of a false hypothesis. This is generally set at a minimum of $80 \%$.

- The intra-cluster correlation coefficient. This is defined as the variance (in the outcome variable) between clusters, divided by (variance between clusters + variance within clusters). Clusters in this case are dormitory schools.

Generally, in impact evaluations of education programs, learning outcomes are measured in terms of standard deviation changes in student test scores. To guide sample size calculations, 27 impact evaluations of education programs carried out by Innovations for Poverty Action were used. Those impact evaluation point to effect sizes ranging from 0.1 standard deviations to 0.3 standard deviations where "an increase of less than 0.1 standard deviations is typically considered to be a small effect, while an increase of more than 0.3 standard deviations is considered a large effect." Within these ranges and within the survey budget, the largest possible sample available was sought.

\section{H. STUDY LIMITATIONS AND CHALLENGES}

A number of challenges were observed during survey design and data collection, as follows:

Sample size for the full treatment group. The relatively small number of project treatment dormitories constitute some limitation on the effect that can be detected. Power and sample size calculations show that, for the purpose of measuring impact of the "full treatment" on test scores, there is little chance of detecting any changes unless there is a very large effect.

- Over-registration of dormitory students. According to the financing policy of the Ministry of Education, Culture, Science and Sports, dormitories are financed by the state budget based on a per student cost, incentivizing dormitories to present inflated numbers of registered students.

5 As described in A. Banerjee, P. Glewwe, S. Powers, and M. Wasserman. 2013. Expanding Access and Increasing Student Learning in Post-Primary Education in Developing Countries: A Review of the Evidence. Abdul Latif Jameel Poverty Action Lab (J-PAL) Post-Primary Education Initiative Review Paper. 
The baseline survey team found this in the majority of dormitories, which in turn affected the number of actual students enrolled and, hence, the number that was available for interviews at these dormitories.

Seasonal fluctuation in student attendance. Many dormitories experience seasonal fluctuations in their number of resident students particularly among primary students. This is because some herder families build an additional ger at the soum center during the winter to accommodate their children in school. Generally, husband looks after their livestock outside of soum center and wife stays close to school at soum center, together with their children. However, their dormitory-registered children are still counted as a student in that dormitory even if she or he is staying at home during some semesters of the school year.

- Difficulty reaching parents. Due to financial constraints for the baseline survey, parents of selected students were interviewed by phone. However, for a variety of reasons, such as parents not owning a phone, being outside cellphone coverage, or no updated phone number being available at the school, a proportion of parents could not be reached. As a result, the number of interviewed parents was lower than the number of surveyed students, which means that it will not be possible to link the parent and student information for a subset of the sample. Information on parents is missing for a total of 735 students.

- Lack of data to verify parallel trends assumption. The validity of the difference-in-differences approach (and as such, the internal validity of the study) hinges on the validity of the parallel trends assumption, which holds that treatment and comparison groups would have developed at the same trend in the absence of the intervention. This assumption can never be directly tested, but data on pre-baseline outcomes can provide an indication of its validity. No such data were available for intermediate and advanced outcomes during the baseline survey. They may be available in the final outcomes at the school level at endline survey.

- Data on key final outcomes-including net intake rate in 1st grade, dropout rates, completion rate, and gross intake rate in the final grade of primary education - could not be presented in the baseline survey report but are expected to be included (for both pre- and post-project years) in the endline survey report.

\section{SURVEY ETHICS}

Informed consent. This was obtained from respondents prior to the interview. Participants were informed about the project, the purpose of the baseline survey, the voluntary nature of participation in the survey, the possibility to skip questions and to end the interview at any time, the confidentiality of identity, and safe and confidential storage and usage of the collected information.

- Consent for student survey. This was collected during the interview with the parents. For a subset of students where parents could not be reached prior to the interview, an agreement was made with class teachers for them to provide information and obtain consent from parents, ex post. 
Confidentiality. To the extent possible, interviews were conducted in private to ensure qualified collection of data and preserve the confidentiality of interviewees.

- Data protection. The survey data were stored at the baseline survey team's server, with no public access. Only the survey design specialist had permission to access the survey database. Other team members, including the quantitative data analyst, requested necessary data through the survey design specialist.

- Privacy protection. All names and personal identification, such as personal ID and addresses were removed from parent and student data and replaced by a nonpersonal survey ID number. Names and other personal identification were stored separately with a note of the nonpersonal ID number so that the datasets can be linked to the personal data when and if needed, such as for identification in the endline survey. 


\section{ANNEX 5 \\ YEAR OF DORMITORY CONSTRUCTION AND RENOVATION}

Table A4: Year of Construction: Full Treatment

\begin{tabular}{|c|c|c|c|}
\hline Year & Number & $\begin{array}{c}\text { Cumulative } \\
\text { (Number) }\end{array}$ & $\begin{array}{c}\text { Cumulative } \\
(\%)\end{array}$ \\
\hline 1957 & 1 & 1 & 3 \\
\hline 1965 & 1 & 2 & 7 \\
\hline 1973 & 1 & 3 & 10 \\
\hline 1974 & 2 & 5 & 17 \\
\hline 1975 & 5 & 10 & 34 \\
\hline 1976 & 1 & 11 & 38 \\
\hline 1978 & 2 & 13 & 45 \\
\hline 1979 & 2 & 15 & 52 \\
\hline 1980 & 2 & 17 & 59 \\
\hline 1985 & 2 & 19 & 66 \\
\hline 1986 & 1 & 20 & 69 \\
\hline 1987 & 1 & 21 & 72 \\
\hline 1988 & 1 & 22 & 76 \\
\hline 1989 & 2 & 24 & 83 \\
\hline 2009 & 2 & 26 & 90 \\
\hline 2010 & 2 & 28 & 97 \\
\hline 2016 & 1 & 29 & 100 \\
\hline
\end{tabular}

Source: Baseline survey conducted by the authors.
Table A5: Year of Renovation: Full Treatment

\begin{tabular}{c|c|c|c}
\hline Year & Number & $\begin{array}{c}\text { Cumulative } \\
\text { (Number) }\end{array}$ & $\begin{array}{c}\text { Cumulative } \\
(\%)\end{array}$ \\
\hline 2002 & 3 & 3 & 15 \\
\hline 2004 & 1 & 4 & 20 \\
\hline 2005 & 1 & 5 & 25 \\
\hline 2006 & 2 & 7 & 35 \\
\hline 2008 & 1 & 8 & 40 \\
\hline 2009 & 1 & 9 & 45 \\
\hline 2010 & 3 & 12 & 60 \\
\hline 2012 & 3 & 15 & 75 \\
\hline 2013 & 1 & 16 & 80 \\
\hline 2014 & 1 & 17 & 85 \\
\hline 2015 & 2 & 19 & 95 \\
\hline 2016 & 1 & 20 & 100 \\
\hline
\end{tabular}

Source: Baseline survey conducted by the authors. 
Table A6: Year of Construction: Light Treatment

\begin{tabular}{|c|c|c|c|}
\hline Year & Number & $\begin{array}{l}\text { Cumulative } \\
\text { (Number) }\end{array}$ & $\begin{array}{c}\text { Cumulative } \\
(\%)\end{array}$ \\
\hline 1960 & 1 & 1 & 2 \\
\hline 1964 & 1 & 2 & 4 \\
\hline 1970 & 2 & 4 & 8 \\
\hline 1971 & 4 & 8 & 15 \\
\hline 1973 & 2 & 10 & 19 \\
\hline 1974 & 1 & 11 & 21 \\
\hline 1975 & 1 & 12 & 23 \\
\hline 1976 & 1 & 13 & 25 \\
\hline 1977 & 1 & 14 & 26 \\
\hline 1978 & 3 & 17 & 32 \\
\hline 1979 & 3 & 20 & 38 \\
\hline 1980 & 3 & 23 & 43 \\
\hline 1981 & 3 & 26 & 49 \\
\hline 1982 & 1 & 27 & 51 \\
\hline 1983 & 3 & 30 & 57 \\
\hline 1984 & 2 & 32 & 60 \\
\hline 1985 & 3 & 35 & 66 \\
\hline 1986 & 1 & 36 & 68 \\
\hline 1987 & 1 & 37 & 70 \\
\hline 1988 & 2 & 39 & 74 \\
\hline 1989 & 1 & 40 & 75 \\
\hline 1990 & 3 & 43 & 81 \\
\hline 1991 & 1 & 44 & 83 \\
\hline 1996 & 1 & 45 & 85 \\
\hline 2005 & 1 & 46 & 87 \\
\hline 2011 & 3 & 49 & 92 \\
\hline 2014 & 1 & 50 & 94 \\
\hline 2015 & 1 & 51 & 96 \\
\hline 2016 & 2 & 53 & 100 \\
\hline
\end{tabular}

Source: Baseline survey conducted by the authors.
Table A7: Year of Renovation: Light Treatment

\begin{tabular}{c|c|c|c}
\hline Year & Number & $\begin{array}{c}\text { Cumulative } \\
\text { (Number) }\end{array}$ & $\begin{array}{c}\text { Cumulative } \\
(\%)\end{array}$ \\
\hline 1996 & 1 & 1 & 3 \\
\hline 2003 & 2 & 3 & 8 \\
\hline 2004 & 2 & 5 & 13 \\
\hline 2005 & 3 & 8 & 21 \\
\hline 2007 & 1 & 9 & 24 \\
\hline 2008 & 2 & 11 & 29 \\
\hline 2009 & 6 & 17 & 45 \\
\hline 2010 & 4 & 21 & 55 \\
\hline 2011 & 1 & 22 & 58 \\
\hline 2012 & 2 & 24 & 63 \\
\hline 2013 & 4 & 28 & 74 \\
\hline 2014 & 1 & 29 & 76 \\
\hline 2015 & 6 & 35 & 92 \\
\hline 2016 & 3 & 38 & 100 \\
\hline $50 u c 15$ & & \\
\hline
\end{tabular}

Source: Baseline survey conducted by the authors. 
Table A8: Year of Construction: Comparison

\begin{tabular}{|c|c|c|c|}
\hline Year & Number & $\begin{array}{l}\text { Cumulative } \\
\text { (Number) }\end{array}$ & $\begin{array}{c}\text { Cumulative } \\
(\%)\end{array}$ \\
\hline 1962 & 1 & 1 & 1 \\
\hline 1970 & 2 & 3 & 4 \\
\hline 1971 & 1 & 4 & 5 \\
\hline 1973 & 5 & 9 & 11 \\
\hline 1974 & 4 & 13 & 16 \\
\hline 1975 & 2 & 15 & 19 \\
\hline 1976 & 1 & 16 & 20 \\
\hline 1977 & 1 & 17 & 21 \\
\hline 1978 & 5 & 22 & 27 \\
\hline 1979 & 2 & 24 & 30 \\
\hline 1980 & 3 & 27 & 33 \\
\hline 1981 & 1 & 28 & 35 \\
\hline 1982 & 3 & 31 & 38 \\
\hline 1983 & 6 & 37 & 46 \\
\hline 1984 & 3 & 40 & 49 \\
\hline 1985 & 3 & 43 & 53 \\
\hline 1986 & 5 & 48 & 59 \\
\hline 1987 & 1 & 49 & 60 \\
\hline 1989 & 3 & 52 & 64 \\
\hline 1990 & 3 & 55 & 68 \\
\hline 1995 & 1 & 56 & 69 \\
\hline 1996 & 1 & 57 & 70 \\
\hline 1997 & 1 & 58 & 72 \\
\hline 2001 & 1 & 59 & 73 \\
\hline 2002 & 1 & 60 & 74 \\
\hline 2003 & 1 & 61 & 75 \\
\hline 2004 & 1 & 62 & 77 \\
\hline 2006 & 1 & 63 & 78 \\
\hline 2007 & 1 & 64 & 79 \\
\hline 2009 & 1 & 65 & 80 \\
\hline 2010 & 3 & 68 & 84 \\
\hline 2011 & 1 & 69 & 85 \\
\hline 2012 & 2 & 71 & 88 \\
\hline 2013 & 2 & 73 & 90 \\
\hline 2014 & 3 & 76 & 94 \\
\hline 2015 & 2 & 78 & 96 \\
\hline 2016 & 3 & 81 & 100 \\
\hline
\end{tabular}

Source: Baseline survey conducted by the authors.
Table A9: Year of Renovation: Comparison

\begin{tabular}{|c|c|c|c|}
\hline Year & Number & $\begin{array}{c}\text { Cumulative } \\
\text { (Number) }\end{array}$ & $\begin{array}{c}\text { Cumulative } \\
(\%)\end{array}$ \\
\hline 1987 & 1 & 1 & 2 \\
\hline 1998 & 1 & 2 & 4 \\
\hline 2003 & 2 & 4 & 8 \\
\hline 2004 & 1 & 5 & 10 \\
\hline 2005 & 3 & 8 & 16 \\
\hline 2006 & 7 & 15 & 29 \\
\hline 2007 & 3 & 18 & 35 \\
\hline 2008 & 4 & 22 & 43 \\
\hline 2009 & 6 & 28 & 55 \\
\hline 2010 & 4 & 32 & 63 \\
\hline 2011 & 1 & 33 & 65 \\
\hline 2012 & 3 & 36 & 71 \\
\hline 2013 & 3 & 39 & 76 \\
\hline 2014 & 6 & 45 & 88 \\
\hline 2015 & 2 & 47 & 92 \\
\hline 2016 & 3 & 50 & 98 \\
\hline 2017 & 1 & 51 & 100 \\
\hline 5017 & 5 & \\
\hline
\end{tabular}

Source: Baseline survey conducted by the authors. 


\section{ANNEX 6 \\ VIGNETTES}

B1. Smiley faces used by students during interview for expressing their sentiments and well-being.
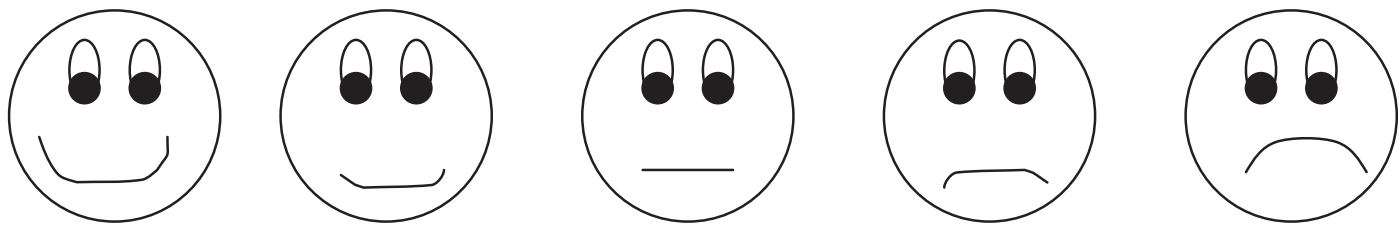

These drawings were borrowed from the survey tools of the study described in Yue et al. 2012. Dormitory Management and Boarding Students in China's Rural Elementary Schools. REAP Working Paper 242. https://papers.ssrn.com/sol3/papers.cfm?abstract_id=2937676.

\section{B2. Anchoring vignettes used for measuring child well-being}

V1 How well/happy do you generally feel? (Use smiley face)

Very unhappy

Unhappy

Neither happy nor unhappy

Somewhat happy

Very happy
1

2

3

4

5

Now I am going to describe the situation of three different school children. For each of them I would like you to answer the question "How well/happy does he/she feel?"

V2.1 Tuya sleeps well at night and usually never feels cold, uncomfortable or hungry at the dormitory. She goes to class every day and when she finishes school she plays with her friends or does her homework. There is a space in the dormitory to do her homework. She feels safe around the guards and the dormitory teacher. She likes living in the dormitory.

V2.2 How well/happy does Tuya generally feel? (Use smiley face)

Very unhappy

Unhappy

Neither happy nor unhappy

Somewhat happy

Very happy
1

2

3

4

5 
V3.1 Naraa sleeps very badly on most nights. She is often cold and feels uncomfortable. She is scared to wake up or go to the bathroom at night. She is often sick or feels sad when she is at the dormitory. There are a lot of fighting and quarreling at the dormitory. The guards and the teacher are not nice to the kids.

V3.2 How well/happy does Naraa generally feel? (Use smiley face)

Very unhappy 1

Unhappy 2

Neither happy nor unhappy 3

Somewhat happy 4

Very happy 5

V4.1 Most nights, Bold sleeps well, but sometimes when the weather is bad outside, he is cold and feels uncomfortable sleeping in the dormitory. He tries to do his homework after school, but sometimes it is difficult to find a quiet place to do it. He gets along well with his friends, but the teacher and guards are not always friendly, and he does not want to go to them if he feels sad.

V4.2 How well/happy does Bold generally feel? (Use smiley face)

Very unhappy

1

Unhappy 2

Neither happy nor unhappy 3

Somewhat happy 4

Very happy

V4 Now, compared to Tuya, Naraa, and Bold, can you tell me how well/happy do you feel?

More than Tuya

Like Tuya

More than bold but less than Tuya

Like Bold

More than Naraa but less than Bold

Like Naraa

Less than Naraa
1

2

3

4

5

6

7 


\section{ANNEX 7}

\section{SELECTED INDICATORS ACROSS TREATMENT AND COMPARISON GROUPS}

Table A10: Prevalence of Selected Indicators across Treatment and Comparison Groups at Baseline

\begin{tabular}{|c|c|c|c|c|c|c|}
\hline & $\begin{array}{c}\text { Full } \\
\text { Treatment }\end{array}$ & $\begin{array}{c}\text { Full } \\
\text { Comparison }\end{array}$ & $\begin{array}{l}\text { Light } \\
\text { Treatment }\end{array}$ & $\begin{array}{l}\text { Light } \\
\text { Comparison }\end{array}$ & $\begin{array}{c}\text { All } \\
\text { Treatment }\end{array}$ & $\begin{array}{c}\text { All } \\
\text { Comparison }\end{array}$ \\
\hline \multicolumn{7}{|l|}{ Respondent background } \\
\hline Herder father & 94 & 89 & 93 & 87 & 93 & 88 \\
\hline Herder legal guardian & 31 & 44 & 43 & 45 & 38 & 45 \\
\hline Father ethnicity & 99 & 99.5 & 99 & 99.8 & 99 & 100 \\
\hline Father-no education & 22 & 10 & 22 & 15 & 22 & 13 \\
\hline Mother-no education & 16 & 6 & 15 & 8 & 16 & 8 \\
\hline Parents' average income (MNT) & $6,874,651$ & $7,676,392$ & 7,547,455 & $7,789,929$ & 7,295,796 & $7,754,470$ \\
\hline Female schoolteacher & 97 & 99 & 94 & 98 & 95 & 98 \\
\hline Female dormitory teacher & 71 & 73 & 73 & 78 & 73 & 77 \\
\hline Female guard & 100 & 88 & 79 & 83 & 86 & 84 \\
\hline Female principal & 33 & 54 & 47 & 64 & 43 & 60 \\
\hline \multicolumn{7}{|l|}{ Dormitory characteristic } \\
\hline Roof leaking & 20 & 22 & 12 & 13 & 15 & 15 \\
\hline Wind blowing through windows & 33 & 24 & 23 & 23 & 26 & 23 \\
\hline Children with own bed & 53 & 60 & 59 & 54 & 57 & 56 \\
\hline Indoor latrine & 21 & 41 & 41 & 38 & 35 & 39 \\
\hline Individual door in toilet facility & 58 & 64 & 61 & 71 & 60 & 69 \\
\hline Bin for hygiene products & 25 & 41 & 30 & 38 & 29 & 39 \\
\hline A quiet place to study in the dormitory & 43 & 54 & 56 & 57 & 51 & 56 \\
\hline \multicolumn{7}{|l|}{ Student well-being } \\
\hline Feeling cold in winter & 16 & 10 & 10 & 13 & 12 & 12 \\
\hline Missed school day in the last 2 weeks & 17 & 16 & 13 & 16 & 15 & 16 \\
\hline $\begin{array}{l}\text { Missed school day in the last } 2 \text { weeks } \\
\text { due to not liking school }\end{array}$ & 4 & 6 & 2 & 2 & 3 & 3 \\
\hline Sick in the last 2 weeks & 20 & 19 & 13 & 17 & 16 & 18 \\
\hline Like classes "very much" & 85 & 81 & 87 & 86 & 86 & 84 \\
\hline
\end{tabular}




\section{Continued}

\begin{tabular}{l|l|l|l|l|l} 
Full & Full & Light & Light & All & All \\
\hline
\end{tabular}

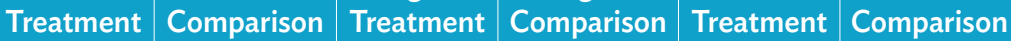

\section{Dormitory teachers}

Training for teacher-professional development to improve teaching qualifications

Training on student-centered methodology

Training on child violence and dealing with conflicts among students

\section{Dormitory Cooks}

Training on meal preparation for students

Training on food nutritional value

Training on food safety

\begin{tabular}{|c|c}
79 & 86 \\
\hline 33 & 27 \\
\hline 54 & 41 \\
\hline
\end{tabular}

\begin{tabular}{|c|}
55 \\
32 \\
50
\end{tabular}

$:$

73
22
42

\begin{tabular}{|c|c|}
\hline 63 & 77 \\
\hline 33 & 23 \\
\hline 51 & 42 \\
\hline
\end{tabular}

\begin{tabular}{l|l|l} 
& 19 & 48 \\
29 & 29 \\
29 & 29
\end{tabular}

\begin{tabular}{l|l}
48 & \\
29 & \\
29 &
\end{tabular}

\begin{tabular}{l:|}
40 \\
\hdashline 58
\end{tabular}

\begin{tabular}{c|c|c}
42 & 34 & 44 \\
\hline 36 & 49 & 34 \\
\hline 42 & 41 & 38
\end{tabular}

\begin{tabular}{l|l|l|l|l|l|l|}
\hline Guards & 12 & 17 & 23 & 19 & 19 & 18 \\
\hline Training for dormitory guards & 19 & 13 & 15 & 13 & 16 & 13 \\
\hline $\begin{array}{l}\text { Training on student-centered communication } \\
\text { methodology }\end{array}$ & & 27 & 29 & 26 & 40 & 27 \\
\hline $\begin{array}{l}\text { Training on child violence and dealing with } \\
\text { conflicts among students }\end{array}$ & & & & \\
\hline
\end{tabular}

\begin{tabular}{|c|c|c|c|c|c|c|}
\hline \multicolumn{7}{|l|}{ Parents' participation } \\
\hline Parents' meeting & 49 & 58 & 51 & 52 & 50 & 53 \\
\hline Average weekly hours of parental support & 3.3 & 3.3 & 3.5 & 2.8 & 3.4 & 3.0 \\
\hline $\begin{array}{l}\text { Parents reporting sending their children to } \\
\text { dormitory because of good physical environment }\end{array}$ & 22 & 29 & 21 & 27 & 21 & 28 \\
\hline \multicolumn{7}{|l|}{ Others } \\
\hline Number of meals served daily & 4.3 & 4 & 4.3 & 4.2 & 4.3 & 4.1 \\
\hline Dormitories with student committees & 96 & 100 & 93 & 94 & 94 & 96 \\
\hline Net intake rate in 1 st grade & 0.90 & 0.86 & 1.00 & 0.93 & 0.90 & 0.90 \\
\hline Net enrollment rate in 1st grade (2017-2018) & 0.74 & 0.81 & 0.89 & 0.9 & 0.84 & 0.88 \\
\hline Dropout rate & 0 & 0.001 & 0.001 & 0.0013 & 0.0006 & 0.0013 \\
\hline Dropout number & 0 & 6 & 11 & 15 & 11 & 21 \\
\hline Average math score & 72 & 66 & 71 & 71 & 72 & 64 \\
\hline Average language score & 74 & 66 & 72 & 73 & 73 & 68 \\
\hline Average science score & 76 & 68 & 74 & 74 & 75 & 68 \\
\hline
\end{tabular}

Note: All reported numbers are percentages unless otherwise indicated. 


\section{ANNEX 8 \\ PHOTOS}

\section{CEILINGS AND WINDOWS}

Students reported feeling cold in winter, leaking dormitory ceilings, and wind blowing through dormitory windows (photos by the authors).
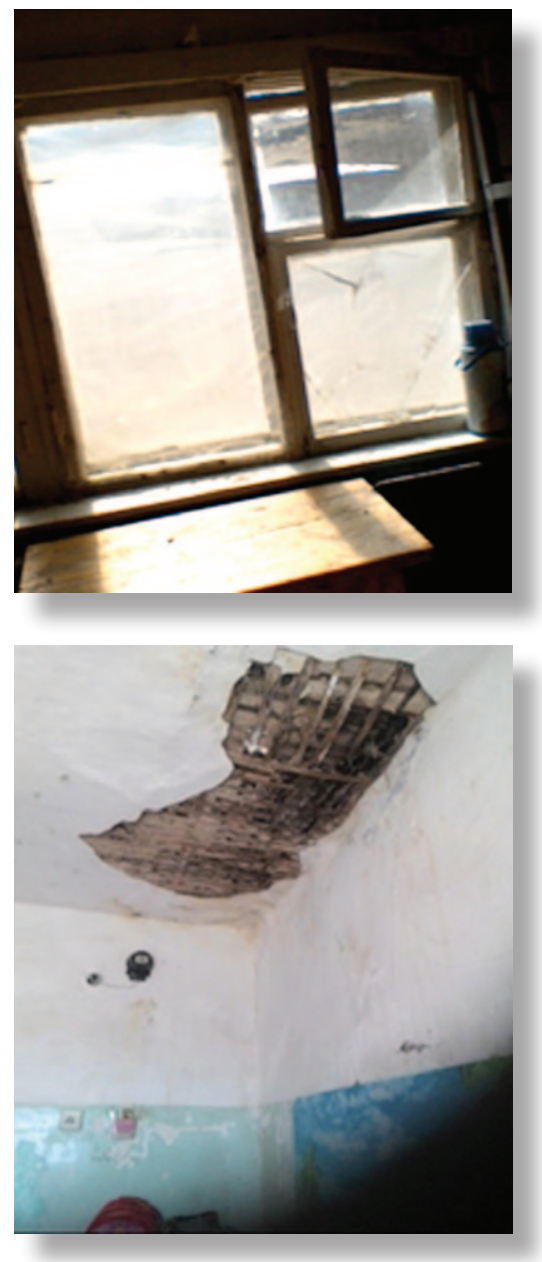
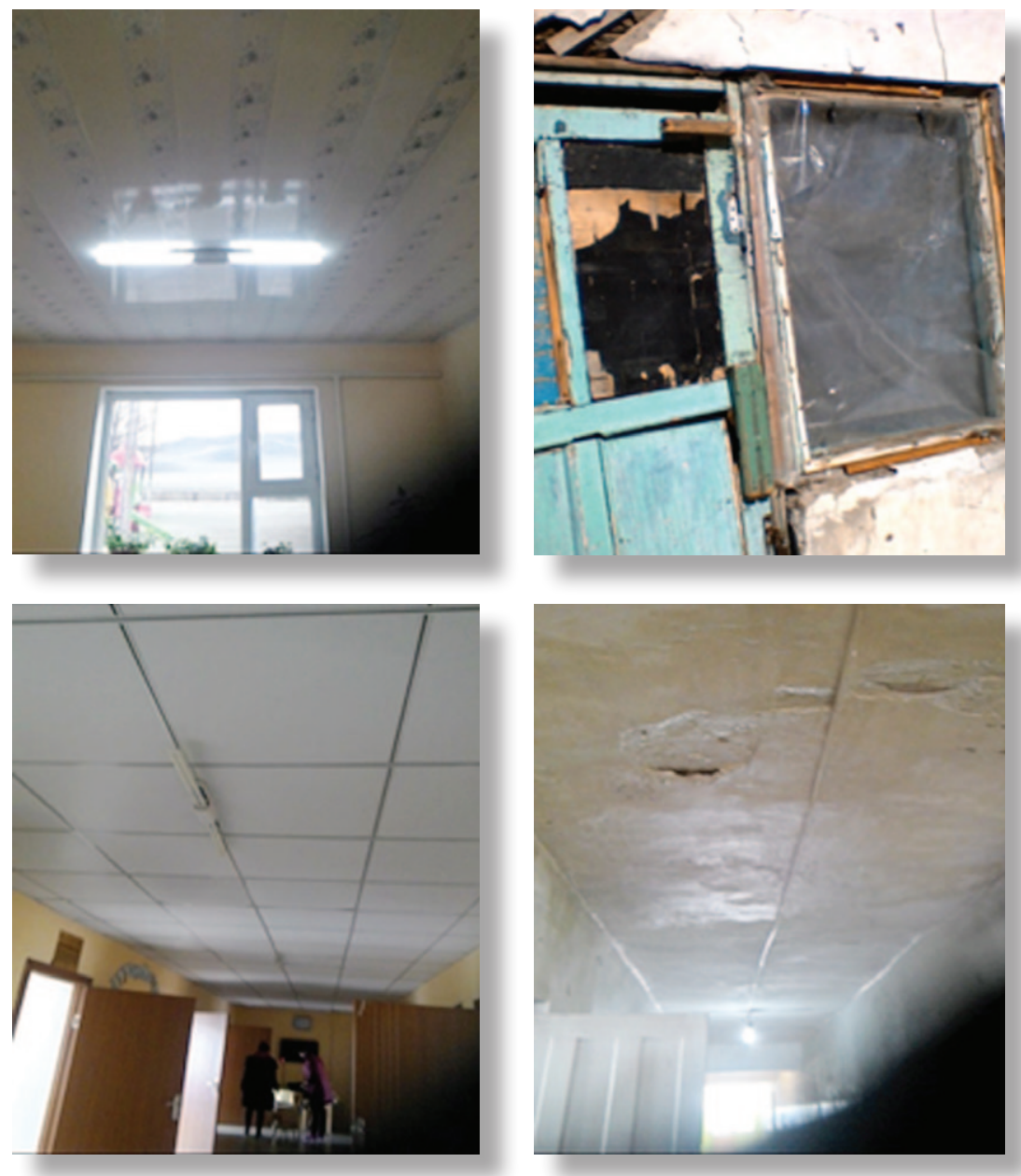
Many indoor and outdoor latrines were rated unsuitable for early-grade students and students with disabilities by enumerators (photos by the authors).
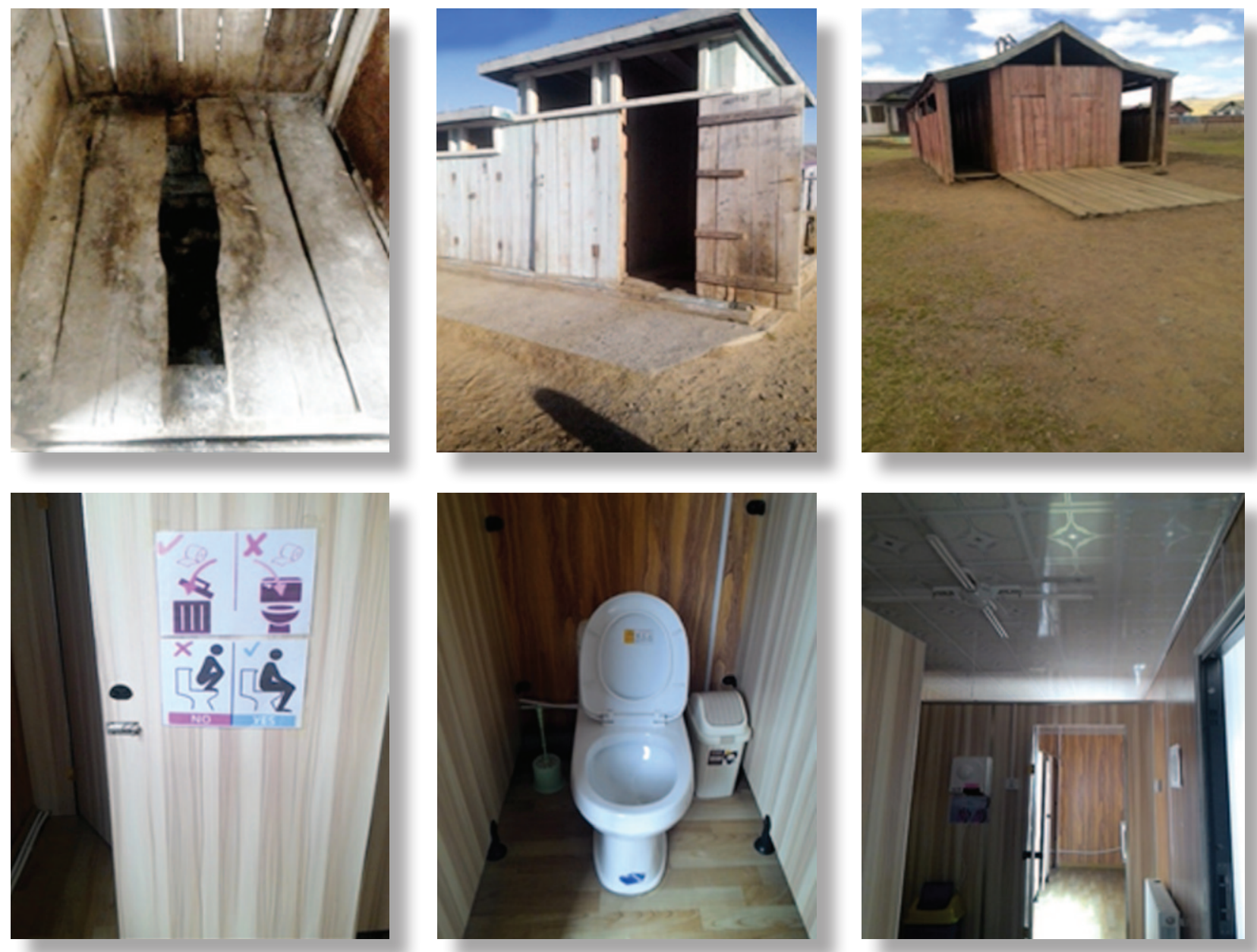


\section{SEPARATE TOILETS FOR GIRLS AND BOYS}

Although most latrines for girls and boys were separated by walls, not all of them had individual doors or locks (photos by the authors).
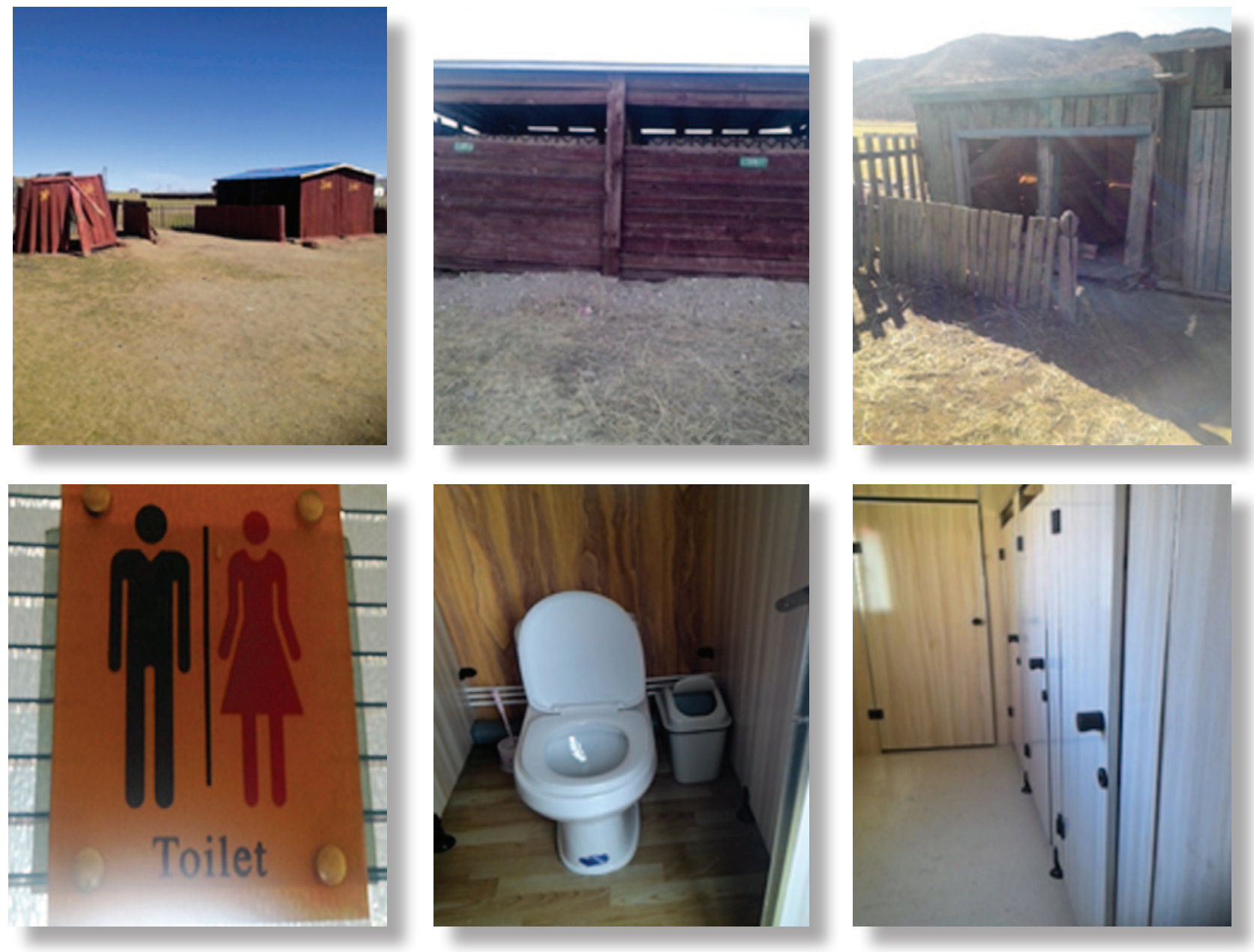


\section{TOILET FACILITIES FOR GIRLS}

The condition of toilets for girls were worst at dormitories in the full treatment group (photos by the authors).
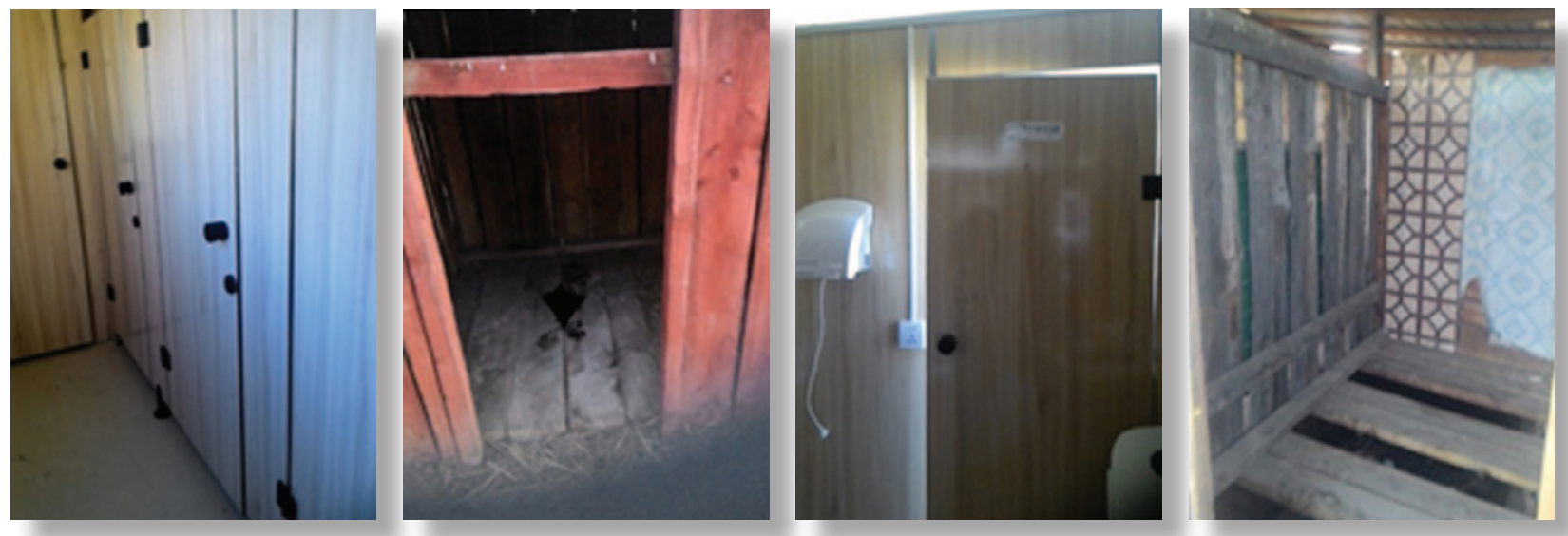

5. SINKS

Tap water was unavailable in most dormitories, affecting the students' hygiene practices (photos by the authors).
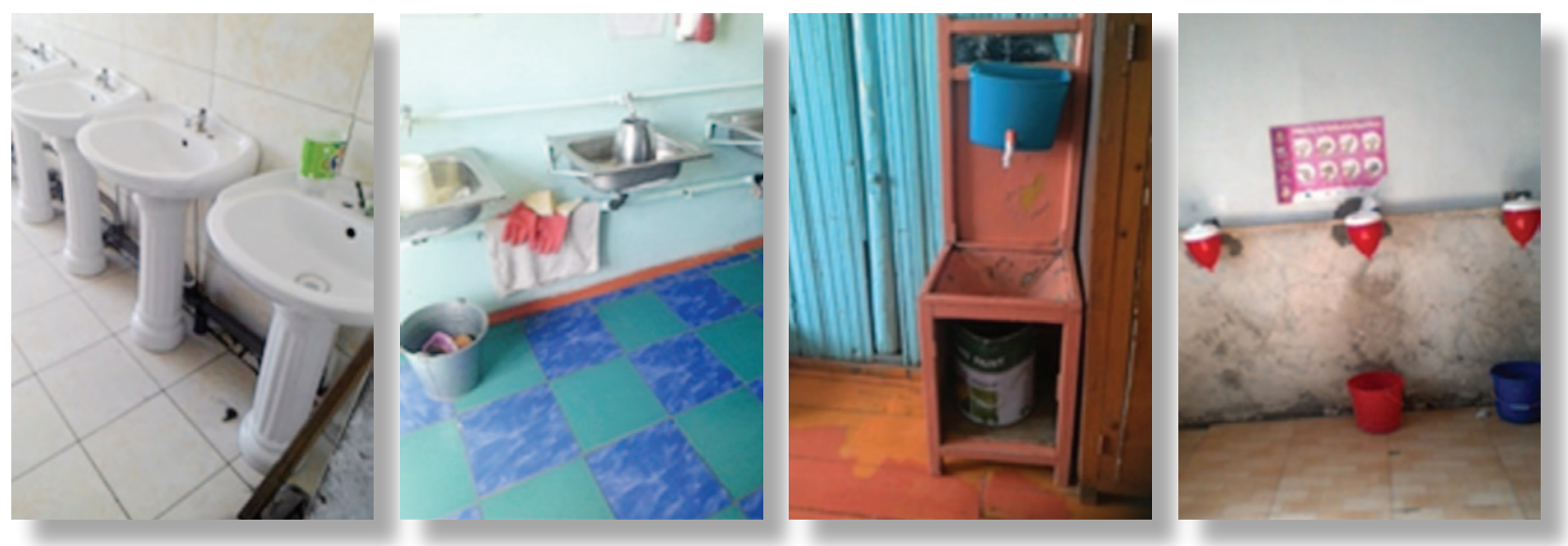


\section{BEDS}

Where beds were insufficient, students needed to share a bed (photos by the authors).
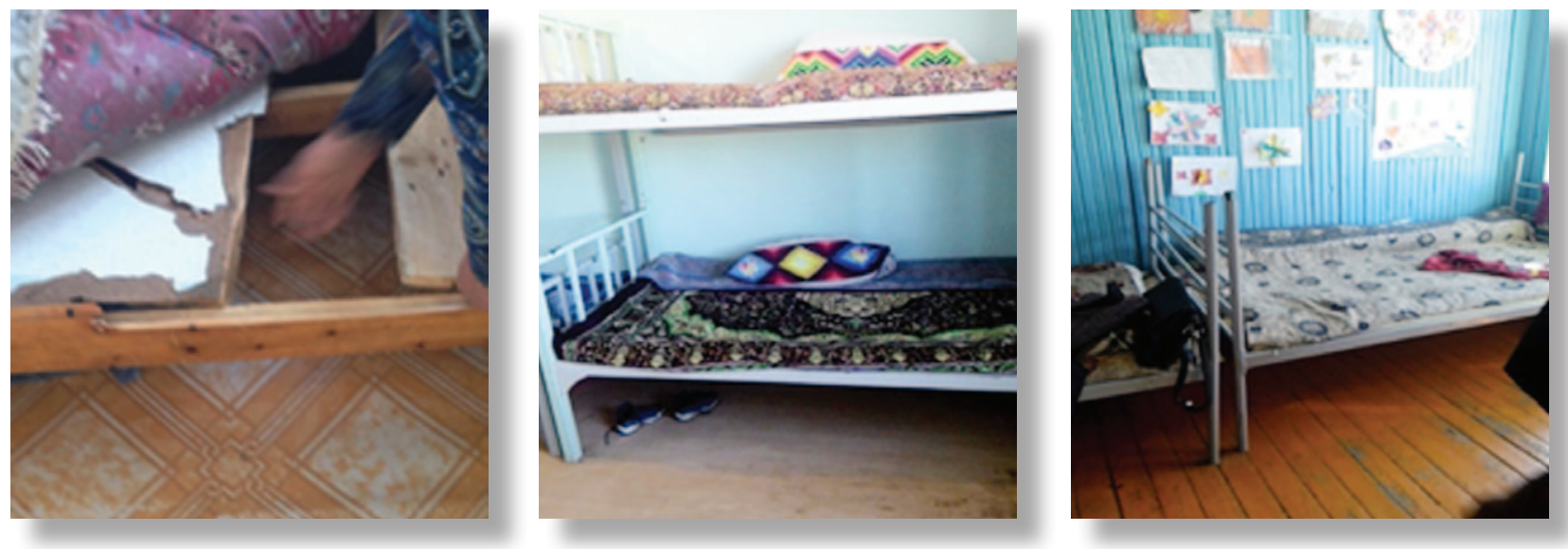

7. FIRE SAFETY PLAN, MAP, AND FIRE FIGHTING EQUIPMENT

Only 9.5\% of dormitories in the full treatment group were equipped with fire safety measures, compared to $44 \%$ in the light treatment group, and $59 \%$ in the comparison group (photos by the authors).
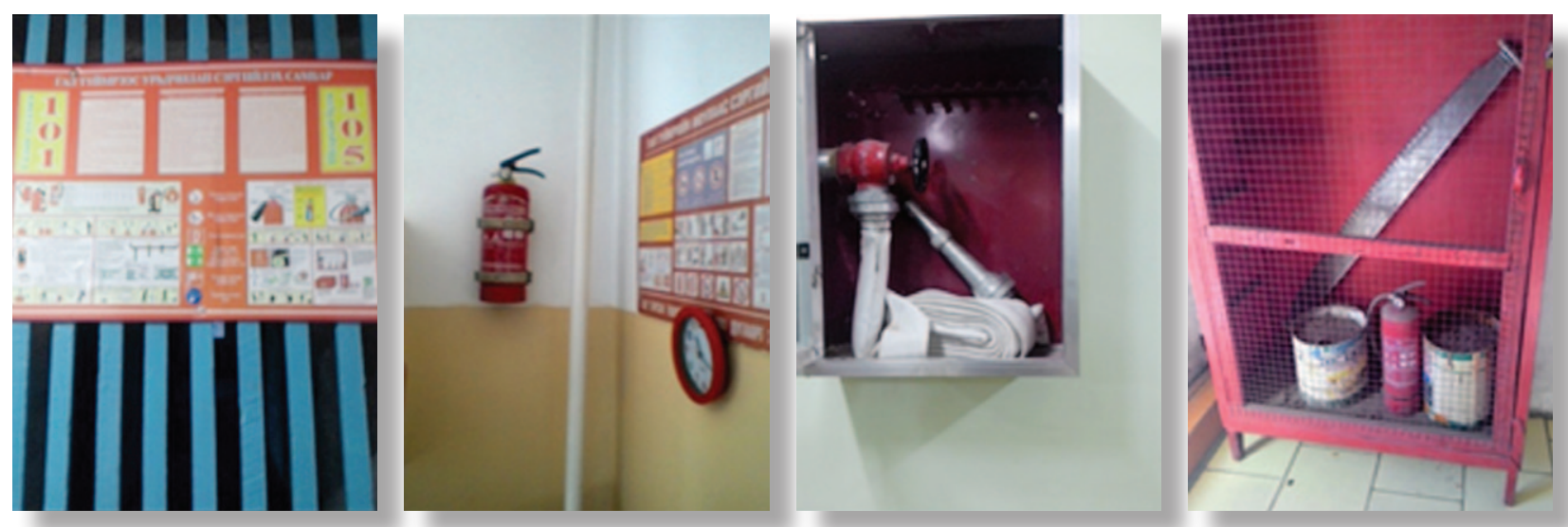


\section{Impact Evaluation Baseline Survey of School Dormitory Environment in Mongolia}

This report presents the impact evaluation of a grant project administered by the Asian Development Bank to improve the school dormitory environment for primary students in western Mongolia. It describes the methodology of the impact evaluation and discusses the findings of the baseline survey of dormitories, schools, and circumstances surrounding survey respondents during the school year 2016-2017. The survey also covered school attendance, levels of participation in study, reading and extracurricular activities, and 5 th grade test scores among primary students living in dormitories. Recommendations are provided for preparing an endline survey to measure impacts of the grant project in the school year 2020-2021.

\section{About the Asian Development Bank}

ADB is committed to achieving a prosperous, inclusive, resilient, and sustainable Asia and the Pacific, while sustaining its efforts to eradicate extreme poverty. Established in 1966, it is owned by 68 members

-49 from the region. Its main instruments for helping its developing member countries are policy dialogue, loans, equity investments, guarantees, grants, and technical assistance. 